\title{
FACTOR VIII INHIBITORS IN HAEMOPHILIA A
}

\author{
Min Ling (M. B., B. S.) \\ Division of Haematology \\ Institute of Medical and Veterinary Science
}

and

Department of Clinical and Experimental Pharmacology

The University of Adelaide

A thesis submitted in partial fulfilment of the requirements for the degree of

Master of Medical Science

November, 2000 


\section{TABLE OF CONTENTS}

DECLARATION iv

ACKNOWLEDGMENTS $\mathrm{v}$

SUMMARY viii

INDEX OF FIGURES xi

INDEX OF TABLES xii

ABBREVIATIONS xiii

CHAPTER ONE: INTRODUCTION AND AIMS

1. Haemophilia A 1

1.1 Pathogenesis 1

1.2 Clinical Presentation 2

1.3 Treatment 3

1.4 Acquired Haemophilia A 6

2. Factor VIII 7

2.1 Coagulation Pathway $\quad 7$

$\begin{array}{ll}2.2 \text { Role of Factor VIII in Blood Coagulation } & 10\end{array}$

2.3 Human Factor VIII Molecule 11

2.4 Factor VIII Gene 13

3. Factor VIII Inhibitors 13

$\begin{array}{ll}3.1 \text { Incidence } & 13\end{array}$

$\begin{array}{ll}3.2 \text { Risk Factors } & 14\end{array}$

3.3 Characteristics of Factor VIII Inhibitors 16

$\begin{array}{ll}3.4 \text { Detection of Factor VIII Antibodies } & 17\end{array}$

$\begin{array}{ll}3.5 \text { Kinetic Classification of Factor VIII Inhibitors } & 18\end{array}$

3.6 Treatment of Inhibitor Patients 19

$\begin{array}{ll}3.7 \text { Acquired Factor VIII Inhibitors } & 21\end{array}$

4. In Vitro Kinetic Study of the Two Types of Factor VIII Inhibitors 21

4.1 Kinetic Features of Factor VIII Inhibitors 22

4.2 Different Patient Groups with Different Antibody Kinetic Types 24 
5. Methods to Detect Anti-Factor VIII Antibodies

5.1 Bethesda Assay to Quantitate Inhibitor Titre 25

5.2 ELISA Method to Detect Anti-Factor VIII Antibodies 27

6. Epitope Detection on Factor VIII Peptides 30

7. Aims of the Project 32

CHAPTER TWO: MATERIALS AND METHODS

1. Patient and Control Samples 34

$\begin{array}{ll}1.1 \text { Patients } & 34\end{array}$

1.2 Sample Preparation 34

1.3 Normal Plasma Used in ELISA Assay 35

2. Materials 35

2.1 Bethesda Assay and Modifications for Kinetic Studies 35

$\begin{array}{ll}2.2 \mathrm{IgG} \text { Purification from Plasma } & 37\end{array}$

2.3 ELISA Method to Detect Factor VIII Antibody 38

2.4 Epitope Detection on Factor VIII Peptide $\quad 40$

3. Methods 46

3.1 In Vitro Kinetics of Factor VIII Inhibitors 46

3.2 ELISA Method to Detect Anti-Factor VIII Antibodies 49

3.3 Epitope Detection on Factor VIII Peptide $\quad 50$

CHAPTER THREE: IN VITRO KINETICS OF FACTOR VIII INHIBITORS

1. Introduction 56

$\begin{array}{ll}\text { 2. Methods } & 60\end{array}$

3. Results 61

3.1 Effect of Incubation Time $\quad 61$

3.2 Antibody Concentration Study 63

3.3 Effect of Extra Source of Factor VIII 68

3.4 Effect of Initial Factor VIII Concentration $\quad 70$

4. Chapter Discussion $\quad 71$

5. Chapter Summary 80 
CHAPTER FOUR: ELISA METHOD TO DETECT FACTOR VIII ANTIBODIES

1. Introduction 82

2. Methods 84

2.1 Establishment of ELISA to Detect Factor VIII Antibody 84

2.2 Patient Group $\quad 85$

2.3 Different Factor VIII Products 85

3. Results 86

3.1 The Normal Range $\quad 86$

3.2 Comparison of Results Between ELISA and Bethesda Assay 86

3.3 Different Factor VIII Products to Set up the ELISA Method 87

4. Chapter Discussion 89

5. Chapter Summary 93

CHAPTER FIVE: EPITOPE DETECTION ON FACTOR VIII PEPTIDE

1. Introduction 95

2. Methods 96

3. Results 97

3.1 Thrombin-Cleaved Fragments of Recombinant Factor VIII 97

3.2 Bacterial Vector Expression of Factor VIII Fragment A1 99

4. Chapter Discussion 101

5. Chapter Summary 103

$\begin{array}{ll}\text { CHAPTER SIX: GENERAL DISCUSSION } & 105\end{array}$

$\begin{array}{ll}\text { REFERENCES } & 115\end{array}$ 


\section{DECLARATION}

This work contains no material which has been accepted for the award of any other degree or diploma in any university or tertiary institution and, to the best of my knowledge and belief, contains no material previously published or written by another person, except where due reference has been made in the text.

I give consent to this copy of my thesis, when deposited in the University Library, being available for loan and photocopying.

Min Ling 


\section{ACKNOWLEDGMENTS}

There are many people to whom I wish to express my gratitude for their help and support during the two years of my study here, which has been a very exciting and unforgettable experience in my life.

First and foremost, I would sincerely like to thank my supervisor, Dr John Lloyd, for his continuous guidance, advice, support and dedication throughout the duration of this project. Thank you not just for your supervision or for giving me the chance to taste the research side of medicine, but also for teaching me to think like a scientist. It has been a privilege to be your student.

My extreme and ineffable gratitude goes to Mrs Elizabeth Duncan, for her relentless and invaluable guidance and advice, and for her never-ending support and friendship. This work would not have been possible without her encouragement and dedication. She is one of the best supervisors and friends I have ever known. Thank you so much for everything Liz.

I am sincerely grateful to Mrs Susan Rodgers, for her constant and invaluable advice, discussion and encouragement since day one. Not only was she there ready to answer my questions whenever I needed, but she has always generously provided her help and patience endlessly. A heart felt thankyou, Sue!

A very big thankyou to the staff of the Haemostasis section, Cheryl, Oriana, Thet and Sonya, for making my time here enjoyable, and for their understanding, patience, and lots 
of help. You are all wonderful people. Special thanks to Eleanor Brown, for her fun, inspiration, help and friendship, and for allowing me to bother her with all different kinds of questions (not scientific, but English and cultural!). It had been a fantastic time to have her companionship.

I would also like to thank:

Gordon Crabb, for his technical assistance in the mathematical analysis of the kinetic data, and for his continuous help.

A/Prof Andrew Somogyi for his expertise on presentation and explanation of the kinetic results.

Karen Ambler, for her patience and technical skills in teaching and helping me with molecular biology experiments.

Stuart Read, for his generous time and useful discussions on both the technical and theoretical advice of some molecular biology and immunology work.

Tony Cambereri for his immunology expertise.

Denise Barbulescu, for offering kind and timely help upon the completion of the thesis. Molecular Pathology Division of the IMVS, for allowing me to work in their laboratory. Haematology Division of the IMVS and Pharmacology Department of the University, for their administrative work throughout my candidature.

Especially, thank you to all my friends: Shoo Peng for giving me support in many different ways; Liu Li, Gao Lu, Eric, Grace, Emma, Cindy, Connie and David for their great friendship and encouragement; Michael and Mark for their inspiration. Finally, I would like to express my deepest gratitude to my family for their love and support. Mum 
and Dad thank you for giving me the opportunity to fly, for believing in me, and above all, your tireless guidance through thick and thin. 


\section{SUMMARY}

Factor VIII inhibitors (antibodies) can arise in patients with inherited haemophilia A as an immune response to infused factor VIII, or can cause acquired haemophilia $\mathrm{A}$ in individuals with a previously normal factor VIII level. Two different types of factor VIII inhibitors form, with different kinetics. Type I inhibitors inactivate all factor VIII available, and usually develop in inherited haemophilia A. Type II inhibitors have more complex kinetics; not all factor VIII is neutralised, and they are usually seen in acquired haemophilia. Fields of interest for the study of these inhibitors include the mechanisms of inactivation of factor VIII coagulant activity (FVIII:C) and determination of their binding sites (epitopes). Research concerning such scientific interests may also have practical application purposes in the future, in terms of improving the therapeutic strategies of haemophilia patients with inhibitors.

The first part of my project focused on the in vitro kinetics of factor VIII inhibitors, aiming to re-examine the available methodology for characterising the kinetics of inhibitor binding to factor VIII, and to evaluate their practicality and sensitivity. Antibody concentration studies, time course studies and alternative approaches were assessed, using modifications of the Bethesda assay to measure the effect of inhibition. Statistical analyses were performed, including mathematical modelling of kinetic data. My work highlighted difficulties in comprehension, execution and interpretation of results using previously described methods. During the course of the project a simple and practical approach to classify inhibitors was developed. The main conclusion was that the use of plasma dilution studies was a readily standardised method to distinguish type I inhibitors from type II. Other strategies to classify inhibitors were less successful. In 
cases with a low-titre inhibitor $(<10 \mathrm{BU} / \mathrm{ml})$, purified and concentrated $\mathrm{IgG}$ was necessary to enable classification. In our study group of 11 patients ( 5 severe, 3 mild and 3 acquired), eight type I and three type II inhibitors were found using a combination of both observation and mathematical methods. This is the first time that a systematic study of methods to evaluate in vitro kinetics of factor VIII inhibitors has been performed to classify inhibitor types in a small but diverse group of patients with haemophilia A.

The second aim of the project was to establish and evaluate ELISA methodology to detect factor VIII antibodies. This method has the ability to detect antibodies which do not inhibit the function of FVIII:C, as well as those that do. Using the ELISA method, some workers showed a very high prevalence of antibodies in patients with severe inherited haemophilia A with the intron 22 inversion of the factor VIII gene. Therefore, specific attention was paid to comparing the ELISA method with the conventional Bethesda assay, and to determining whether there was any difference in the prevalence of antibodies in severe patients with and without the intron 22 inversion. Samples with a wide range of inhibitor titres by Bethesda assay were studied, as well as samples from a group of haemophilia patients with no detectable inhibitor. Formulated recombinant factor VIII was used to coat the micro-titre plates, and a range of plasma dilutions from both normal individuals and patients was tested. The conclusion was that in our study group of 50 haemophilia A patients, the ELISA method revealed a level of sensitivity for detection of factor VIII antibodies that was similar to the Bethesda assay. In addition, ELISA showed that the prevalence of antibodies in severe patients with intron 22 gene inversion was similar to that in those without the inversion. An ELISA method using unformulated recombinant factor VIII has also been established, and this will be used to 
screen a number of patients to give more information about this method and its sensitivity for detecting factor VIII antibodies.

The third aim of the project was to investigate methods for epitope mapping of the factor VIII molecule. Thrombin-cleaved recombinant factor VIII was studied by Western blotting to detect fragments which bind to monoclonal antibodies. The heavy chain and its activation/inactivation products from thrombin cleavage were demonstrated to bind to monoclonal anti-heavy chain antibody MAS 530. Experimental conditions to detect binding of patient antibodies need to be optimised. A bacterial expression system to produce A1 fragment was attempted but was not successful. Evidence was obtained to show that the vector gene (with the A1 gene) was inserted into E. Coli cells. However it was not possible to express the protein (A1 fragment) using this system. Although the methodology remains a challenge, using recombinant DNA technology to produce fragments of factor VIII of any desired size and section of the molecule still seems to be a reasonable way to carry out a thorough study of epitope determination.

The work done for this project has initiated the study of a possible relationship between the epitope specificity, genetic defect, and clinical and kinetic features of factor VIII inhibitors. Future studies may give additional information about the mechanisms of antibody formation and their inactivation of FVIII:C, which may lead not only to more informed and effective therapeutic approaches for haemophilia patients with inhibitors, but also to a better understanding of the human immune response. 


\section{INDEX OF FIGURES}

Figure

Title

Page No*

1.1 A schematic diagram of some of the clinically important 8 coagulation reactions

1.2 Structural domains and epitope regions in factor VIII 12

$\begin{array}{lll}1.3 & \text { Gene organisation and processing of factor VIII } & 12\end{array}$

3.1 Time course graphs for type I kinetics 57

3.2 Time course graph for type II kinetics 57

3.3 Effect of incubation time (Case 1, Du, BU=30) 61

3.4 Effect of incubation time (Case 9, Si, BU=140) 61

3.5 Effect of incubation time (Case 11, Mi, BU=3) 61

3.6 Effect of antibody concentration (Cases 1-6) 64

3.7 Effect of antibody concentration (Cases 7-11) 64

3.8 Antibody concentration study using purified and concentrated $\operatorname{IgG} 65$

$3.9 \quad$ Study of effect of extra source of factor VIII 69

$3.10 \quad$ Further study of effect of extra factor VIII 69

3.11 Study of effect of initial factor VIII concentration (four cases) 71

3.12 Study of effect of initial factor VIII concentration (Case 1) 71

4.1 ELISA binding curves for two patients and two normal controls 86

4.2 ELISA results in patient groups with or without Bethesda titre $\quad 86$

4.3 The correlation between the Bethesda titre and the OD value in 87

4.4 Three different factor VIII products to coat the microtitre plates 88 in ELISA method

5.1 Time course of thrombin cleavage of recombinant factor VIII 98 detected by Western blot, using two monoclonal antibodies

5.2 Western blot for thrombin-cleaved recombinant factor VIII, using 98 anti-heavy chain (MAS 530) and anti-light chain (MAS 531) monoclonal antibodies

5.3 Western blot for thrombin-cleaved recombinant factor VIII, using 98 monoclonal antibody (MAS 530) and human IgG concentrates

5.4 Western blot for thrombin-cleaved unformulated recombinant 99 factor VIII (Baxter), using monoclonal antibodies MAS 530 and ESH 8

$\begin{array}{lll}\text { 5.5 PCR products for the Al gene } & 100\end{array}$

5.6 Colony screening for the insertion of A1 gene into the vector 100

$\begin{array}{lll}5.7 & 100\end{array}$

*: page number preceding the figure. 


\section{INDEX OF TABLES}

Figure

Title

Page No*

3.1 Clinical data of 11 haemophilia A patients in the kinetic study $60 \dagger$

3.2 Comparison of time course results from the two phase

63

exponential decay model analysis with the kinetic classification by antibody concentration study on 9 o f 11 patients

3.3 IgG purification and concentration of six plasma samples

3.4 Results of antibody concentration study from the sigmoidal dose-response (variable slope) model analysis on 11 patients

3.5 Summary of 11 haemophilia A patients in the in vitro kinetic studies

4.1 Summary of 50 haemophilia A patients in the ELISA study to detect factor VIII antibodies

4.3 Comparison of the two studies on factor VIII antibody detection in patients with severe haemophilia

*: page number preceding the table, except where otherwise indicated $(\dagger, t)$.

$\dagger$ : facing page, landscape table.

\$: on page. 


\section{ABBREVIATIONS}

IIa activated factor II

VIIa activated factor VII

VIIIa activated factor VIII

IXa activated factor IX

$\mathrm{Xa} \quad$ activated factor $\mathrm{X}$

XIa activated factor XI

XIIa activated factor XII

aa amino acid (residues)

AHF antihaemophilic factor

APCC activated prothrombin complex concentrate

APTT activated partial thromboplastin time

Arg Arginine

B-PN buffered pooled normal (plasma)

BT bleeding time

BU Bethesda unit

$\mathrm{Ca} \quad$ calcium

Cys Cysteine

EC50 effective concentration to induce $50 \%$ of the maximal response

E. Coli Escherichia Coli

ECL enhanced chemiluminescence

ELISA enzyme linked immuno-sorbent assay

FVIII:C factor VIII procoagulant/coagulant activity

FVIII:Ag factor VIII procoagulant antigen 


\begin{tabular}{|c|c|}
\hline FS & full strength \\
\hline GST & glutathione S-transferase \\
\hline $\mathrm{HCl}$ & hydrochloric acid \\
\hline His & Histidine \\
\hline HMWK & high-molecular-weight kininogen \\
\hline HRP & horse radish peroxidase \\
\hline $\operatorname{IgG}$ & immunoglobulin class $\mathrm{G}$ \\
\hline IPTG & isopropyl-beta-D-thiogalactoside \\
\hline IU & international unit \\
\hline $\mathrm{kb}$ & kilo base \\
\hline $\mathrm{KD}$ & kilo Dalton \\
\hline OD & optical density \\
\hline OPD & 1,2-phenylenediamine dihydrochloride \\
\hline PAGE & polyacrylamide gel electrophoresis \\
\hline PBS & phosphate buffered saline \\
\hline PCC & prothrombin complex concentrate \\
\hline PCR & polymerase chain reaction \\
\hline PF3 & platelet factor 3 \\
\hline PK & prekallikrein \\
\hline PL & phospholipid \\
\hline $\mathrm{PN}$ & pooled normal (plasma) \\
\hline PT & prothrombin time \\
\hline rFVIII & recombinant factor VIII \\
\hline SD & standard deviation of series \\
\hline
\end{tabular}


SDS sodium dodecyl sulphate

$\mathrm{TF} \quad$ tissue factor

TF:VIla tissue factor-activated factor VII complex

TFPI tissue factor pathway inhibitor

Tyr Tyrosine

vWF von Willebrand factor 


\section{CHAPTER ONE}

\section{INTRODUCTION AND AIMS}

In this review of the literature on factor VIII inhibitors in haemophilia A, specific attention has been paid to the three selected features, namely, the in vitro kinetics of factor VIII inhibitors, the immunoassay of factor VIII antibodies, and the detection of epitopes on the factor VIII peptide.

\section{Haemophilia A}

\subsection{Pathogenesis}

The word haemophilia is defined by The Oxford English Dictionary as "a constitutional (usually hereditary) tendency to bleeding, either spontaneously or from very slight injuries; haemorrhagic diathesis" (Simpson and Weiner, 1989). This disease was known to the ancient world, with the earliest written references in Jewish texts of the second century AD (Ingram, 1997). The first modern description of haemophilia is attributed to Dr. John Conrad Otto, who clearly appreciated, in his publication in 1803, the three cardinal features of haemophilia: an "inherited" tendency of "males" to "bleed" (Ingram, 1997). However, the biochemical basis of haemophilia was not elucidated until the early 1950 's, when it was demonstrated that some proteins from normal plasma would correct the abnormal clotting of haemophilic blood (Aggeler et al., 1952; Pavlovsky, 1947). Most of the patients with haemophilia were found to be missing the protein known as antihaemophilic factor (AHF), and the disease was termed haemophilia A. Some patients with identical clinical and genetic features were found to be lacking a different protein, Christmas factor, and their disorder was termed haemophilia B. Standardised names were assigned to the various coagulation factors by the International Committee on Thrombosis 
and Haemostasis in 1962: the factor missing in haemophilia A was subsequently termed factor VIII, and the factor missing in haemophilia B was termed factor IX (Wright, 1962).

Haemophilia $\mathrm{A}$ is the most common form and accounts for about $80 \%$ of the two main forms of the haemophilias associated with a coagulation factor deficiency or defect. Thus the unqualified term haemophilia is classically understood to refer to haemophilia A (Brettler and Levine, 1993). Haemophilia A is described as an inherited blood coagulation disorder caused by the absence or insufficient amount of functional factor VIII, a plasma protein which is essential for blood to clot (Brettler and Levine, 1993; Handin, 1998 a; Mosher, 1996). This recessive, X-linked genetic disease affects approximately one out of 10,000 males in the population, and is found to exist in all races with a similar incidence in all areas of the world (Brettler and Levine, 1993; Hoyer 1994; Levine, 1987). It is known that a great variety of factor VIII gene mutations, located on the X chromosome, are responsible for the aetiology of the lack or deficient activity of clotting factor VIII (Hoyer, 1981; Lozier and High, 1990; Hoyer, 1994; Tuddenham et al., 1994).

\subsection{Clinical Presentation}

The bleeding symptoms with haemophilia A usually occur during childhood and then continue into adult life. The clinical manifestation in severe cases is characterised by spontaneous and recurrent bleeding into soft tissues, muscles, and weight-bearing joints, notably the knees, ankles, elbows and shoulders. The repeated presence of blood in joints will subsequently lead to haemarthrosis and then severe joint deformity, which is known as disabling haemophilic arthropathy. This, together with internal bleeding such as oropharyngeal and central nerve system bleeding, accounts for the apparently reduced life 
expectancy for haemophilia A patients before effective replacement therapy was introduced at the end of the 1960's (Aledort, 1976; Biggs, 1977; Eyster et al., 1978). The laboratory investigations usually reveal a prolonged activated partial thromboplastin time (APTT), with other tests such as platelet count, bleeding time (BT) and prothrombin time (PT) being normal. Specific bioassays to quantitate factor levels are essential to distinguish haemophilia A from haemophilia B (Handin, 1998 a; Mosher, 1996).

Normal haemostasis requires a minimal level of $25-30 \%$ of factor VIII coagulant activity (FVIII:C) in plasma, with a normal range of FVIII:C found to be around 50\%-200\% $(0.50$ 2.00 International Units (IU)/ml plasma) (Brettler and Levine, 1993; Handin, 1998 a). However, symptoms usually occur when the FVIII:C levels are $5 \%$ or less, with a close relation between the clinical severity of haemophilia and plasma FVIII:C level. There are three clinical phenotypes of this disease observed and classified: severe haemophilia with less than $1 \%$ of FVIII:C $(<0.01 \mathrm{IU} / \mathrm{ml})$ and frequent episodes of spontaneous haemorrhage; moderate haemophilia with the plasma FVIII:C between 1-5\% (0.01-0.05 IU/ml) and haemorrhage provoked by slight trauma; and mild haemophilia with the FVIII:C over 5\% $(>0.05 \mathrm{IU} / \mathrm{ml})$ and infrequent bleeding that is usually secondary to major trauma (Brettler and Levine, 1993; Furie et al., 1994; Handin, 1998 a; Mosher, 1996).

\subsection{Treatment}

\subsubsection{Replacement therapy}

A remarkable evolution in haemophilia A replacement therapy has occurred over the past few decades. More than 30 years ago, the treatment for bleeding episodes was by the infusion of whole blood and later frozen plasma, both of which were severely limited 
therapies. With the discovery of concentrated factor VIII in plasma cryoprecipitate by Pool and coworkers in 1964 (Pool et al., 1964), more specific and effective therapy became available. The subsequent continuous development of many novel manufacturing methods for purified factor VIII concentrate has further revolutionised treatment (Aledort, 1996; Hoyer, 1994; Ingram, 1997; Rosendaal et al., 1991).

It has been empirically determined that each unit of factor VIII infused, which is defined as the average amount present in $1 \mathrm{ml}$ normal plasma, will elevate the recipient's factor VIII level in plasma by 0.02 unit per kilogram of body weight. A $70-\mathrm{kg}$ patient with severe haemophilia A requires approximately 3000 units of factor VIII to raise the plasma level to the average of that found in normal subjects $(100 \%$ or $1 \mathrm{IU} / \mathrm{ml})$. As a rule of thumb, infusions of 15, 30 and 60 units per kilogram should raise the plasma level to $25 \%, 50 \%$ and $100 \%$ of normal, respectively (Mosher, 1996). Factor VIII distributes mainly in the blood plasma and the half-life is 10-12 hours (Mosher, 1996).

The amount of factor VIII required depends on the plasma level of factor VIII needed to treat the specific bleeding episode. The distribution and clearance of the factor in plasma should also be taken into consideration (Brettler and Levine, 1993; Handin, 1998 a; Hoyer, 1994; Mosher, 1996). In general, an uncomplicated episode of soft tissue bleeding or an early haemarthrosis can be treated with one infusion of sufficient factor VIII concentrate to raise the factor VIII level to $15 \%$ or $20 \%$ of normal. A more extensive haemarthrosis or retroperitoneal bleeding requires twice or three times-daily or continuous infusions in order to keep the factor VIII level above $25 \%$ for at least 72 hours. Life-threatening 
bleeding or major surgery may require therapy for 2 weeks with levels kept at a minimum of $50 \%$ of normal (Handin, $1998 \mathrm{a}$ ).

With the introduction of prophylactic therapy for haemophilia children from an early age to prevent joint bleeds, and with the availability of home treatment or self-management with factor VIII concentrate injections, the lifestyle for haemophilia A patients has improved significantly in the last decade, with an anticipation of using standard gene therapy treatment to cure the disease in the future (Berntorp et al., 1995; Furie, 1994).

\subsubsection{Complications}

However, with the benefits of replacement therapy of factor VIII, there are also some complications, including viral transmission such as hepatitis $\mathrm{B}$, hepatitis $\mathrm{C}$, and human immunodeficiency virus, and the development of anti-factor VIII antibodies (factor VIII inhibitors) as an immune response to infused factor VIII. The adoption of different virucidal methods during the manufacturing process of plasma-derived factor VIII and genetically-engineered products have offered a better margin of biological safety against viral transmission than in the past (Berntorp et al., 1995; Gilles et al., 1997; Lusher, 1987).

Following multiple transfusions, about $10-20 \%$ of severe haemophilia patients develop inhibitors to factor VIII which neutralise FVIII:C, making the usual doses of replacement therapy ineffective. Patients with inhibitors are usually divided into two basic categories: low responders, whose inhibitor titre usually does not exceed 10 Bethesda units $/ \mathrm{ml}$ $(\mathrm{BU} / \mathrm{ml})$ despite repeated exposure to factor VIII; and high responders, who have an 
anamnestic response to repeated administration of factor VIII, and in whom the inhibitor titre rises above $10 \mathrm{BU} / \mathrm{ml}$ (Roberts and Hoffman, 1995).

In acute situations, patients with inhibitors can sometimes be treated effectively with very high doses of human factor VIII or with porcine factor VIII. As a long-term solution, it is sometimes possible to induce immune tolerance. However, the treatment for such patients is usually problematic and extremely expensive. Therefore, the presence of factor VIII inhibitors is now considered to be a major difficulty in the clinical management of haemophilia A (Berntorp et al., 1995; Gilles et al., 1997; Lusher, 1987).

\subsection{Acquired Haemophilia A}

Autoantibodies directed against factor VIII may also occur spontaneously in individuals with a previously normal FVIII:C level, causing acquired haemophilia A. The incidence of acquired haemophilia $\mathrm{A}$ is thought to be approximately 0.2 to 1.0 per million population, with equal sex prevalence (Green and Lechner, 1981; Shapiro and Hultin, 1975; Shulman and Hirschman, 1969). It may be associated with particular medical conditions, such as pregnancy and the postpartum period, autoimmune disorders, malignancy, and drug allergic reactions, or may be idiopathic, without any identified underlying conditions (Feinstein, 1993; Mosher, 1996; Sallah, 1997). The precise aetiology of inhibitors to factor VIII in these non-haemophilic persons remains obscure.

The spectrum of symptoms in patients with acquired haemophilia A is similar but not identical to that observed in congenital haemophilia. Haemarthrosis, for example, is uncommon in acquired haemophilia, whereas it is the hallmark of congenital (severe) 
haemophilia (Mannucci and Giangrande, 1994). The acquired inhibitors share several features with the factor VIII antibodies seen in hereditary haemophilia, e.g., both are primarily of the immunoglobulin class $\mathrm{G}(\mathrm{IgG})$, and are usually directed against limited epitopes in the factor VIII molecule, destroying coagulant activity. However, there are many differences between these two kinds of inhibitors, including the degree of antibody heterogeneity, reaction kinetics between inhibitor and factor VIII, patient demographics, type and severity of bleeding, natural history, and therapeutic considerations (Lusher, 1995).

\section{Factor VIII}

\subsection{Coagulation Pathway}

Haemostasis is an extremely important biological mechanism that provides a rapid, potent, but tightly localised response to vascular damage. It is comprised of two stages: primary haemostasis and secondary haemostasis. Primary haemostasis includes the processes of blood vessel contraction, platelet adhesion and aggregation immediately after tissue injury. Secondary haemostasis is the process of blood coagulation. It is initiated by substances in injured tissues and propagated by an interlocking network of zymogen-to-proteinase conversions. Blood coagulation results in the formation of a stable fibrin clot that stops further bleeding, thereby allowing subsequent cellular ingrowth and tissue repair to take place (Furie and Furie, 1995; Jesty and Nemerson, 1995; Mosher, 1996).

For in vitro analysis by the clotting tests PT and APTT, the coagulation cascade was divided into two branches: the intrinsic and extrinsic pathway, based on the mechanism by which factor $\mathrm{X}$ is activated. Regardless of which pathway initiates the enzyme-catalysed 
reactions, the two will converge to a common pathway leading to generation of thrombin (activated factor II, or IIa), the master coagulation enzyme (Lozier and High, 1990; Mosher, 1996).

\subsubsection{New concepts about the coagulation pathway}

Recent research shows that the coagulation cascade is a complex interaction between enzymes, and that the primary initiating event in vivo is exposure of tissue factor (TF), and its formation into a complex with activated factor VII (VIIa) (Mann, 1999). TF, the activating substance which is usually found in the tissues rather than in the blood, is a transmembrane glycoprotein expressed on most nonvascular cells and is inducible via de novo synthesis in cells within the blood or on the blood vessel wall, including monocytes and endothelial cells (Furie and Furie, 1995). Upon the formation of the tissue factoractivated factor VII complex (TF:FVIIa) following tissue injury and laceration of blood vessels, the initial product of the complex is factor Xa. Once Xa is generated, it contributes to factor IX activation by a concerted reaction with TF:VIIa. Thus both the intrinsic and extrinsic pathways are initiated (Mann, 1999). According to the new concept about the biochemistry and physiology of blood coagulation, because there are several recognised interactions between the two pathways, it is no longer accurate to view them as separate pathways. However, these two pathways are still measured separately in the in vitro tests. (Figure 1.1)

\subsubsection{Contact activation pathway (formerly known as intrinsic pathway)}

APTT measures the pathway previously known as the intrinsic pathway. In this assay, activation of the pathway occurs when certain coagulation proteins come into contact with 


\section{FIGURE 1.1 A Schematic Diagram of Some of the Clinically Important Coagulation Reactions}

The inactivated or precursor proteins are indicated by roman numbers, and the active form by the addition of a lowercase "a" (a standard convention). Other abbreviations: Ca, calcium; PL, phospholipid; TF: tissue factor. Arrows: those in blue stand for the activation of precursor proteins to their active form; those in red stand for the activation or positive feedback by one factor to another; those in back stand for the inhibition or negative feedback. There are two independent activation pathways, the contact activation pathway (formerly known as intrinsic pathway) and the tissue factor pathway (formerly known as extrinsic pathway). They merge at the point of factor $\mathrm{X}$ activation and lead to the generation of thrombin, which converts fibrinogen into fibrin (not shown on this diagram). In addition, TF-VIIa can activate factor IX which is in the contact activation pathway. These reactions are regulated by a number of negative feedbacks, namely, the inactivation of factor V, VIII, and IX by thrombin, and the inactivation of TF-VII by Xa. Also, the anticoagulant proteins antithrombin III, protein $\mathrm{C}$ and protein $\mathrm{S}$ contribute to inhibition of coagulation (not shown). 


$$
\text { TF + VII }
$$

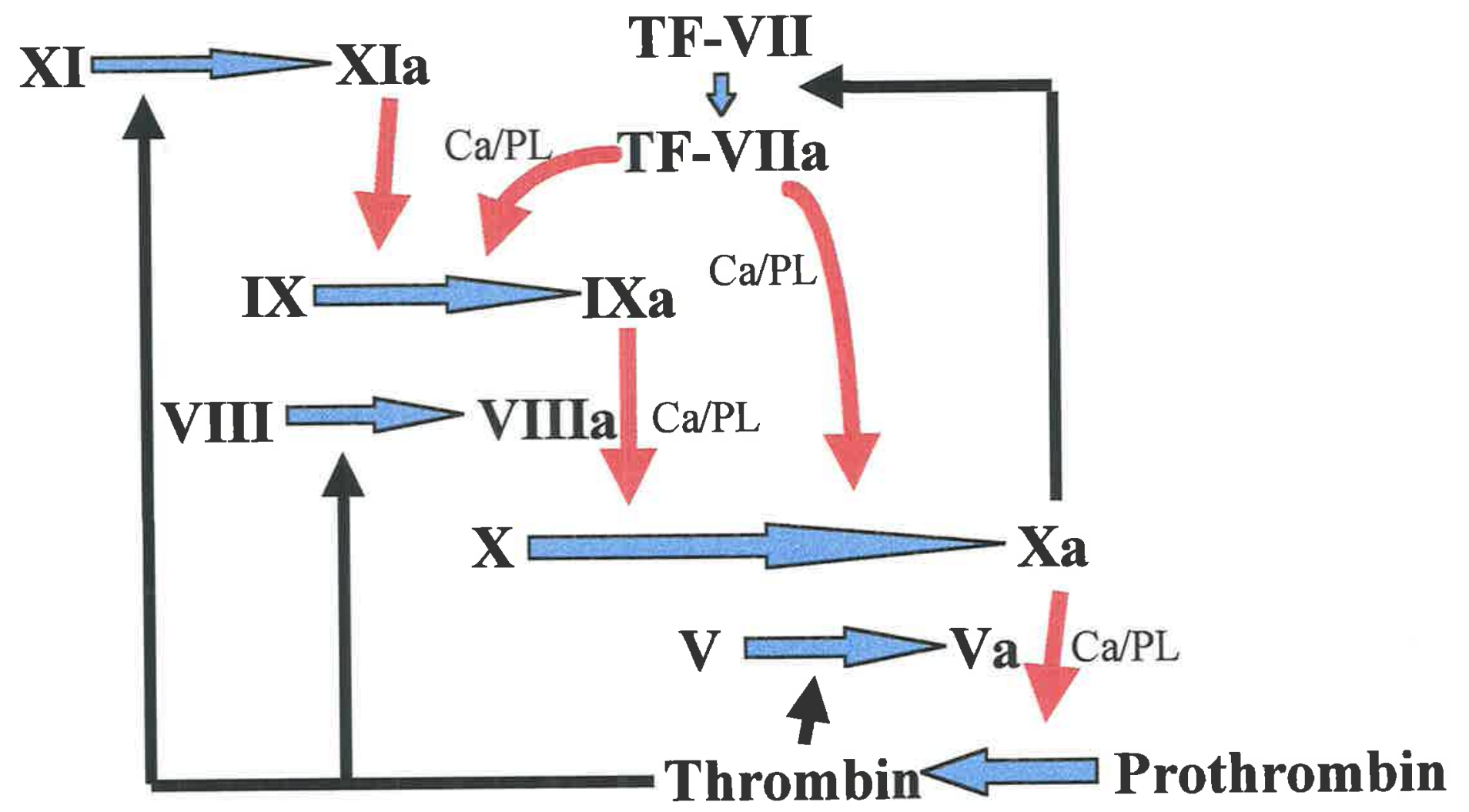


a negatively charged surface (Jesty and Nemerson, 1995; Mosher, 1996). These coagulation proteins are found within the blood (hence the term intrinsic) and are known as contact activation factors. They include prekallikrein (PK), high-molecular-weight kininogen (HMWK), factor XII and factor XI (Mosher, 1996).

Upon contact with a negatively charged surface, the contact activation factors interact. The presence of HMWK, during this interaction, causes the conversion of PK into kallikrein. This, in turn, activates factor XII to form XIIa (the activated state). In the presence of calcium (Ca), XIIa activates HMWK-bound factor XI into XIa (the activated state), which then activates factor IX (Lozier and High, 1990). After this series of reactions, activated factor IX (IXa) combines with activated cofactor VIII (VIIIa) and platelet factor 3 (PF3, a phospholipid supplied by platelets), and in the presence of calcium, it converts factor X of the common pathway to the serine protease $\mathrm{Xa}$ (the activated state). This is followed by subsequent reactions: the activation of factor II to thrombin (IIa) by Xa, then the cleavage of fibrinogen to fibrin by thrombin, and the formation of a blood clot (Jesty and Nemerson, 1995; Lozier and High, 1990; Mosher, 1996). The APTT test is used to assess the factors in the intrinsic and common pathways (Mosher, 1996).

\subsubsection{Tissue factor pathway (formerly known as extrinsic pathway)}

PT measures the pathway previously known as the extrinsic pathway. In this assay, activation occurs following exposure of tissue factor to plasma factor VII and the formation of a TF:VII complex. From this interaction, factor VII is activated to the serine protease VIIa, producing the TF:VIIa complex. This complex, in the presence of calcium and phospholipid (PL), activates factor $\mathrm{X}$ to $\mathrm{Xa}$, causing the subsequent reactions in the 
common pathway. PT measures the factors of the extrinsic and common pathways (Handin, 1998 b; Mosher, 1996).

\subsubsection{Regulation of the coagulation pathway}

For maximum effectiveness, the coagulation pathway is regulated by both positive and negative feedbacks by activated factors such as Xa and IIa, or circulating plasma inhibitors such as Antithrombin III, protein S and protein C, to either accelerate or localise the coagulant reactions (Jesty and Nemerson, 1995). There is also a unique type of negative control via a recently discovered protein, the so-called tissue factor pathway inhibitor (TFPI). It is now thought that factor IX and $\mathrm{X}$ activation by the TF:FVIIa complex plays a major role in the initiation of haemostasis. Once coagulation is initiated by this interaction, TFPI combines with factor Xa, then inhibits the TF:VIIa complex and therefore blocks that pathway. So elements of the intrinsic pathway, particularly factor VIII and IX, become the dominant regulators of thrombin generation (Handin, 1998 b; Jesty and Nemerson, 1995).

\subsection{Role of Factor VIII in Blood Coagulation}

In the blood coagulation cascade, the role of glycoprotein cofactors (factor $\mathrm{V}$ and factor VIII) is essential. After being activated by thrombin, factor VIIIa accelerates the rate of cleavage of factor $\mathrm{X}$ by IXa, causing a 56-fold enhancement of factor IXa activity under physiological conditions (Lozier and High, 1990). A very low concentration of factor VIII $(0.2 \mu \mathrm{g} / \mathrm{ml}$ in plasma) ensures adequate procoagulant function in normal persons; a substantial reduction (to less than $20 \%$ of normal) or absence of factor VIII leads to a bleeding disorder (Hoyer, 1994). 
Factor VIII circulates in the plasma in a noncovalent complex with von Willebrand factor (vWF), a property that protects it from proteolysis, and concentrates it at sites of active haemostasis (Hoyer, 1981; Kaufman et al., 1989; Weiss et al., 1977). The interaction with vWF inhibits FVIII:C. To participate in haemostasis the factor VIII must separate from vWF. This requires the cleavage of factor VIII by thrombin or factor Xa, which enables factor VIII to bind to the phospholipid surfaces of damaged cells and adherent activated platelets, where it dramatically increases the maximal velocity of the enzyme-substrate reaction of factor IXa with factor X, accelerating it several thousandfold (Hoyer, 1981). The effect of the cofactors on activation of factor $\mathrm{X}$ is as follows:

\begin{tabular}{lc}
\hline & Vmax \\
\hline IXa & 0.0022 \\
IXa + Ca & 0.0105 \\
IXa + Ca + PL & 0.0024 \\
IXa + Ca + PL + VIIIa & 500 \\
\hline
\end{tabular}

The factor VIII protein can be identified in two ways, by functional and immunological methods. Factor VIII procoagulant activity (FVIII:C) refers to its functional property in blood coagulation, while factor VIII procoagulant antigen (FVIII:Ag) refers to its antigenic character measured by immunoassays (Hoyer, 1981; Mann et al., 1990; van Dieijen et al., 1981).

\subsection{Human Factor VIII Molecule}

Human factor VIII is a 2351-amino-acid polypeptide from which a hydrophobic signal peptide of 19 amino acids is removed during secretion. Following the signal peptide is the factor VIII protein with the triplicated amino acid domains, A1, A2 and A3 which have 
approximately 40 percent sequence homology with each other. A large B domain separates the $\mathrm{A} 2$ and $\mathrm{A} 3$ domains, and at the carboxyl terminus are two duplicated $\mathrm{C}$ domains (Hoyer, 1994; Lozier and High, 1990). Electron microscopy of rotary-shadowed human factor VIII suggests a configuration of a globular structure $14 \mathrm{~nm}$ in diameter and a B-domain tail that is released by thrombin cleavage (Fowler et al., 1990).

The structural domains of factor VIII occur in the order NH2-A1(amino acid residues 1372)-A2(373-740)-B(741-1689)-A3(1690-2019)-C1(2020-2172)-C2(2173-2332)-COOH.

It is generally accepted that both $\mathrm{A} 2$ and $\mathrm{C} 2$ are important functional domains, as the A2 domain is involved in factor IXa binding, while the A3 and $\mathrm{C} 2$ domains are involved not only in factor IXa binding, but also vWF binding and phospholipid binding (Kaufman and Antonarakis, 1995). (Figure 1.2)

Shortly after synthesis, factor VIII is cleaved during intracellular processing so that plasma factor VIII is a heterodimer: an 80-kilo Dalton (KD) light chain in a divalent, cationdependent complex with a heavy chain that contains one B domain of variable length. On exposure to thrombin or factor $\mathrm{Xa}$, cleavage at $\mathrm{Arg}^{372}$ converts the heavy chain into 54-KD and 44-KD fragments, both of which are essential for procoagulant activity. At the same time, a small fragment is cleaved at $\mathrm{Arg}^{1689}$ to dissociate factor VIII from vWF. The product after these cleavages is the activated factor VIII heterotrimer factor VIIIa, which is composed of the two heavy-chain fragments and the 72-KD light-chain fragment (Hoyer, 1994). (Figure 1.3) 


\section{FIGURE 1.2 Structural Domains and Epitope Regions in Factor VIII}

The mature factor VIII molecule consists of a number of repeating domains in the order A1(aa 1-372)-A2(373-740)-B(741-1689)-A3(1690-2019)-C1(2020-2172)-C2(2173-2332). The important functional regions in the molecule include the A2 domain (factor IX binding), as well as the $\mathrm{A} 3$ and $\mathrm{C} 2$ domains (von Willebrand factor $[\mathrm{vWF}]$ and phospholipid [PL] binding, with factor IX binding as well). The red arrows show the binding sites of factor IX (aa 558-565, 1811-1818), vWF (aa 1649-1689, 2248-2312) and PL(aa 2303-2332) to these functional domains in the factor VIII molecule (major references: Saenko and Scandella, 1995; Saenko et al., 1996; Shima et al., 1995; Zhong et al., 1998). Epitopes recognised by the inhibitors have been located to the three main clusters in the $\mathrm{A} 2, \mathrm{~A} 3$ and $\mathrm{C} 2$ domains, as indicated by the short blue bold lines. aa: amino acid (residues). 


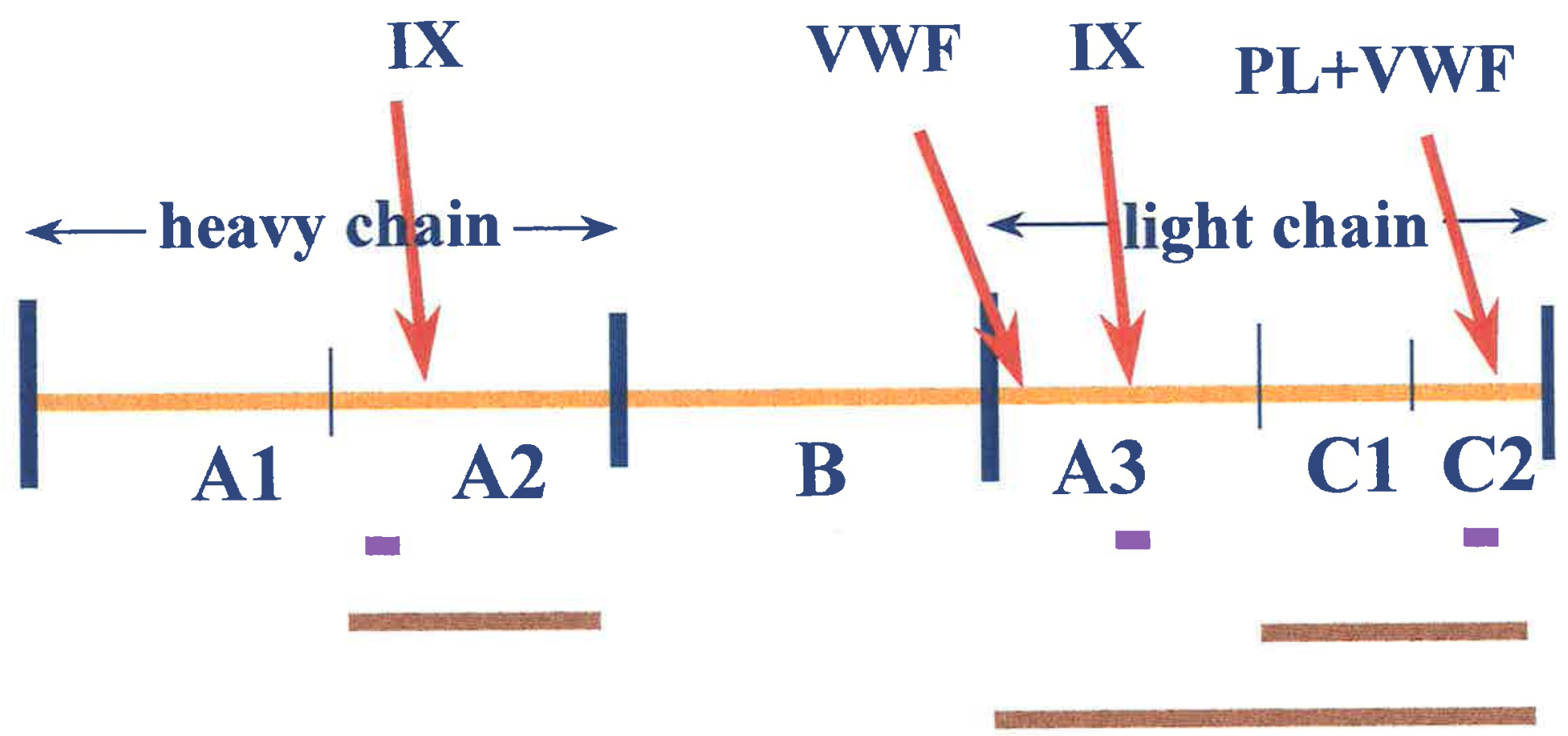




\section{FIGURE 1.3 Gene Organisation and Processing of Factor VIII}

(Reprint from Ichinose and Davie. The Blood Coagulation Factors: Their cDNAs, Genes, and Expression. Hemostasis and Thrombosis: Basic Principles and Clinical Practice. 3rd Edition. 1993. p. 31.)

Top line represents the Factor VIII gene, showing the 26 exons (vertical bars). Factor VIII is synthesized as a single-chain precursor with domains A1, A2, B, A3, C1 and C2. On secretion from the cell, Factor VIII is cleaved into a $200-\mathrm{KD}$ heavy chain and an $80-\mathrm{KD}$ light chain, apparently held together by calcium ions. The $\mathrm{B}$ domain is heavily glycosylated (HGR, heavily glycosylated region). Shaded regions are rich in acidic residues. Arrows indicate the cleavage sites (numbered) by proteolysis during the activation and inactivation of the Factor VIII molecules. 


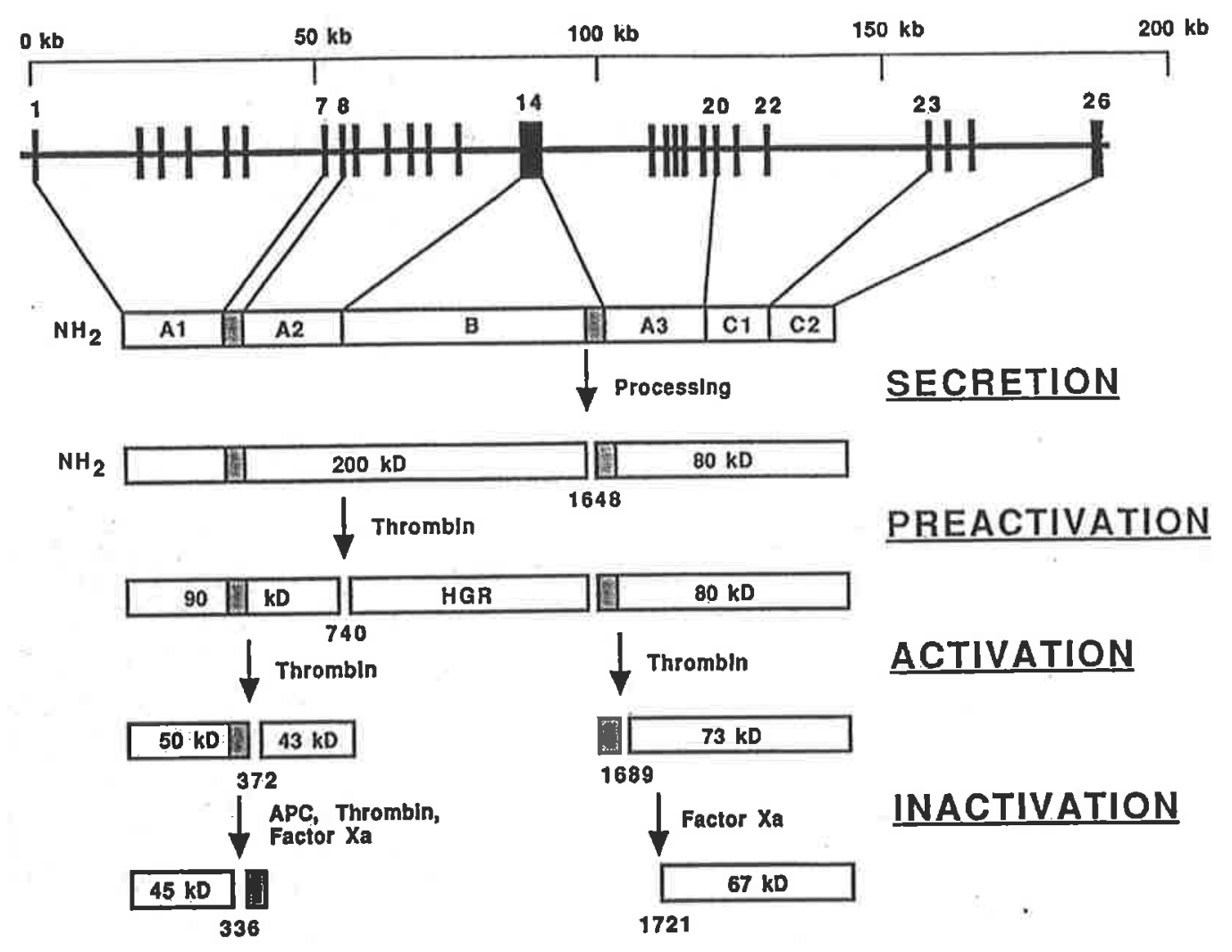




\subsection{Factor VIII Gene}

The factor VIII gene is 186 kilo base $(\mathrm{kb})$ long (approximately $0.1 \%$ of the DNA of the X chromosome) and contains 26 exons and 25 introns. The normal factor VIII mRNA is approximately $9 \mathrm{~kb}$, of which the coding sequence is 7,053 nucleotides. Deleterious mutants in the factor VIII gene result in reduction or absence of the factor VIII protein, and thus cause congenital haemophilia A (Antonarakis et al., 1995). A database of mutations in the factor VIII gene was first set up in 1991 and has been updated through the internet since 1996 (the Haemophilia A Mutation, Search, Test and Resource Site: HAMSTeRS, accessed via the World Wide Web at http://europium.mrc.rpms.ac.uk). Mutations described include single base-pair substitutions, deletions, insertions and rearrangements in the gene (Tuddenham et al., 1991; Tuddenham et al., 1994; Wacey et al., 1996; KemballCook and Tuddenham, 1997).

\section{Factor VIII Inhibitors}

\subsection{Incidence}

An exact figure for the prevalence of factor VIII inhibitors is difficult to provide, as the percentage varies with the population of patients considered, the method used to detect inhibitors, the threshold level and the frequency and time points at which the detection of inhibitors are carried out (Gilles et al., 1997). However, it is generally accepted that inhibitor antibodies to factor VIII develop in 10-20\% of patients with haemophilia A, and more frequently in severe cases, although different studies showed different incidences ranging from $10 \%$ to $45 \%$ (Aledort, 1996; Berntorp et al., 1995; Brettler, 1995; Furie et al., 1994; Gilles et al., 1997; McMillan et al., 1988; Sallah, 1997). Overall, the incidence of inhibitors in patients treated by recombinant factor VIII (rFVIII) is similar to that 
observed in patients on plasma-derived factor VIII preparations, despite the fact that some products did cause much a higher or much lower incidence of factor VIII inhibitors (Bray et al., 1994; Ehrenforth et al., 1992; Gilles et al., 1997; Lusher et al., 1993; Peerlinck et al., 1993).

\subsection{Risk Factors}

It is important to determine the factors that predispose to inhibitor development. This would allow investigation of therapies targeted to prevent the establishment of the inhibitor response or to provide the ability to initiate early immune tolerance induction for those at a risk.

\subsubsection{Nongenetic factors}

The determination of the risk factors has been the subject of a few studies. There are some possible risk factors that are considered to be responsible for the development of factor VIII inhibitors. The nongenetic factors include age, dose and frequency of treatment, and sometimes the particular type of product, which may be something to do with the manufacturing processes (Gill, 1999; Berntorp et al., 1995; Sallah, 1997).

\subsubsection{Genetic factors}

More importantly, genetic or familial factors have long been suspected and confirmed to play a significant role in inhibitor development, in addition to nongenetic factors. In an analysis of 255 severe haemophilic sib-pairs from 54 centres in North America, there were more pairs in which both had inhibitors, than was expected. This was interpreted as supporting the hypothesis that a genetic component to inhibitor formation exists (Gill, 
1999). In a study of 26 mild and moderate haemophilia A patients with inhibitors, it was also suggested there was a familial predisposition to develop inhibitors in the kindreds (Hay et al., 1998). Such a genetic component, however, should be referred to in two parts: both the factor VIII gene mutation and genetic factors other than the factor VIII mutation.

It is easy to understand that the lack of a recognisable factor VIII protein, as a result of a particular genetic abnormality, may be the most important determining factor for antibody formation, as those patients are more likely to see infused factor VIII as a "foreign" protein and therefore develop antibodies. Several studies have shown that in severe haemophilia patients, those with large deletions, stop mutations and intrachromosomal recombinations (intron 22 gene inversion) are more likely to develop inhibitors than those with small deletions and missense mutations (Schwaab et al., 1995; Tuddenham and McVey, 1998). Among these defects, the most common is the gross DNA rearrangement (intron 22 gene inversion) which has been detected in $45 \%$ of patients with severe haemophilia A. In this mutation there is intrachromosomal rearrangement of the tip of the long arm of the X chromosome, with a region within intron 22 of the factor VIII gene (Lakich et al., 1993), which leads to disruption of the gene and absence of factor VIII protein (Lakich et al., 1993). Patients with mild or moderate haemophilia, in which there are measurable levels of factor VIII protein, are much less likely to develop an inhibitor than those with severe haemophilia (Tuddenham and McVey, 1998), possibly because the infused factor VIII is not as readily identified as a foreign protein.

However, not all patients with the intron 22 inversion, large deletions, or nonsense mutations form inhibitors, suggesting that factors other than the factor VIII mutation 
contribute, such as inherited immune-response characteristics of individuals (Antonarakis et al., 1995; Hoyer, 1994). It has been demonstrated that the risk of inhibitor development in the haemophilic sibling of an inhibitor patient is approximately $50 \%$, whereas the risk for an extended family member is only about 10\% (Gill et al., 1996). This finding supports the existence of genetic factors important to inhibitor development, in addition to any role played by the factor VIII mutation. From the studies available so far, a weak association of HLA (human Major Histocompatibility Gene Complex, human MHC) genotype with inhibitor formation has been found (Hay et al., 1997; Oldenburg et al., 1997; Tuddenham and McVey, 1998). In general, studies appear to strongly support the role of genetic factors in the formation of inhibitors, in addition to the specific underlying factor VIII mutation, although some researchers still believe environmental factors may be of the same importance.

\subsection{Characteristics of Factor VIII Inhibitors}

Antibodies to factor VIII are immunoglobulins of the IgG class, most commonly the $\operatorname{IgG}_{4}$ isotype, or a combination of two or more subtypes (Fulcher et al., 1987). Occasional inhibitors that are immunoglobulins of the class A or M have been reported (Gilles et al., 1997; Pruthi and Nichols, 1999). The inactivation of FVIII:C by factor VIII inhibitors is known to be time and temperature dependent, and is irreversible both in vivo and in vitro (Hoyer et al., 1984). In addition, factor VIII inhibitors have some degree of species specificity, especially autoantibodies to human factor VIII, which usually have a much lower cross-reactivity to porcine factor VIII than alloantibodies (Pruthi and Nichols, 1999). 
The main mechanism of inhibition is thought to be by direct interference with factor VIII function. Another mechanism proposed, but not with strong evidence, is that of binding to non-functional regions and increasing the clearance of factor VIII from plasma (Nilsson et al., 1990). Both allo- and autoantibodies are found to be directed mainly towards functional epitopes in the A2 or C2 domains, and inhibit FVIII:C by interfering with thrombin cleavage, factor VIII-vWF interaction, and factor VIII-phospholipid binding (Arai et al., 1989; Lazarchick et al., 1986; Lollar et al., 1997; Shima et al., 1993). Another study found a third main target region located at the amino-terminal end of the A3 domain, where the inhibitors are thought to interfere with vWF binding (Gilles et al., 1993). More recent studies showed that the inhibition by antibodies against this region may also involve the delayed release of factor VIII from vWF, and the impaired binding of factor VIII with factor IX ( Saenko et al., 1996; Zhong et al., 1998).

\subsection{Detection of Factor VIII Antibodies}

The Bethesda method is used in the routine laboratory to quantitate inhibitory anti-factor VIII antibodies (factor VIII inhibitors). It is based on determining the inactivation of FVIII:C in normal plasma, when incubated with inhibitor plasma under specific conditions (Kasper et al., 1975; Verbruggen et al., 1995). One Bethesda unit (BU) is defined as the amount of inhibitor necessary to neutralise $50 \%$ of FVIII:C in normal plasma during incubation for two hours at $37^{\circ} \mathrm{C}$.

Haemophilia patients with factor VIII inhibitors can be divided into two groups, on the basis of the strength of their inhibitor levels after receiving treatment with factor VIII. High responders have a strong anamnestic response and the inhibitor titre increases to $>10$ 
$\mathrm{BU} / \mathrm{ml}$ (or $>5 \mathrm{BU} / \mathrm{ml}$ according to some researchers), whereas low responders have a much weaker response and the titre remains $<10 \mathrm{BU} / \mathrm{ml}$ (or $<5 \mathrm{BU} / \mathrm{ml}$ ) (Berntorp et al., 1995; Feinstein, 1993; Hoyer, 1995; Sallah, 1997). The majority of patients with severe haemophilia who develop inhibitors are high responders, which makes the management of such patients very difficult. Most patients with mild or moderate haemophilia A and patients with autoantibodies to factor VIII are low responders (Sallah, 1997); these inhibitors may be transient, disappearing spontaneously over time (Brettler, 1995; Ehrlich et al., 1998).

In addition to the Bethesda method (based on functional coagulation assays), immunoassays can be used in research laboratories to detect antibodies to factor VIII which may or may not inhibit its function. Several methods based on the immunologic reaction have been developed, including immunoprecipitation, western blot analysis, and enzyme-linked immunosorbent assay (ELISA) technique (Shurafa and Kithier, 1995).

\subsection{Kinetic Classification of Factor VIII Inhibitors}

There are several ways to classify factor VIII inhibitors, e.g., alloantibodies or autoantibodies; high response inhibitors or low response inhibitors. Another classical way to distinguish these inhibitors is by examining the kinetics of their interaction with factor VIII. It is generally accepted that there are two types of factor VIII inhibitors, classified according to their kinetic behaviour: type I inhibitors completely inhibit FVIII:C following second-order kinetics, while type II inhibitors follow more complex kinetics and cannot completely inhibit FVIII:C (Biggs et al., 1972 a and b; Feinstein, 1993; Gawryl and Hoyer, 1982). 
Whatever the precise reason for this difference, the distinction between type I and type II inhibitors remains useful. Type I inhibitors are most often observed in severe haemophilia A patients who respond to factor VIII infusion by producing high titres of antibodies (Gilles et al., 1997). In contrast, type II inhibitors are usually observed in mild or moderate haemophilia A patients, and patients with acquired haemophilia (Gilles et al., 1997; Hoyer and Scandella, 1994; Kasper, 1991). In some patients, a mixture of both type I and type II inhibitors can be found, consistent with the fact that human IgGs are polyclonal antibodies. It has also been demonstrated that some antibodies behaving as type I could be converted to type II upon addition of anti-idiotypic antibodies (Gilles et al., 1997).

\subsection{Treatment of Inhibitor Patients}

Suppressing the production of inhibitors and establishing a state of immune unresponsiveness to factor VIII remain major goals for the treatment of inhibitor patients. We are however, far from reaching such goals, basically due to a poor understanding of the mechanisms underlying specific antibody production and regulation.

Treatment for bleeding episodes by infusion of large doses of factor VIII can be quite effective in patients with low titres of inhibitors and in patients with recent inhibitors (Gilles et al., 1997). The therapeutic options for patients with high-titre inhibitors include the use of materials which bypass the need for factor VIII to allow coagulation, such as administration of porcine factor VIII, prothrombin complex concentrates (PCC), activated PCCS (APPCs), and recombinant activated factor VII (Berntorp et al., 1995; Bloom, 1994; 
Gilles et al., 1997; Sallah, 1997). Usually, a PCC or APCC at a dose of 75 units per $\mathrm{kg}$ is used as first-line treatment for bleeding episodes, although these products are not as effective as is factor VIII for non-inhibitor patients and cannot be relied upon to prevent or control bleeding (Berntorp et al., 1995). More recently, human recombinant activated factor VII has been used with good efficacy (Hedner et al., 1993).

Immune tolerance regimes using factor VIII alone or combined with immunosuppressive drugs (corticosteroids, or cyclophosphamide and intravenous gamma globulin) do have a reasonable success rate in countries which can afford the protocol. Various immunetolerance-induction regimens have been developed and many of them start with large daily doses of factor VIII. While many patients have had good responses, most appear to require prophylaxis in order to maintain suppression of their inhibitor antibody. However, it is strongly recommended that once an inhibitor is diagnosed, immune tolerance should be considered as soon as possible (Berntorp et al., 1995). Plasmapheresis to remove antibodies has limited success.

In general, for haemophilia A patients with antibodies against factor VIII, treatment depends upon the immune response pattern, the antibody titre, and the severity of the haemorrhagic episode. However, the development of novel and more successful clinical strategies for the treatment of inhibitor patients is still a big challenge, which requires a more thorough understanding of the mechanisms by which these inhibitors are formed and their mode of action. 


\subsection{Acquired Factor VIII Inhibitors}

Autoantibodies in patients with acquired haemophilia $\mathrm{A}$ have a number of similar characteristics to the alloantibodies from patients with inherited haemophilia A. For example, there is a predominance of the $\mathrm{IgG}_{4}$ subclass in both groups, in contrast to the very low (about $5 \%$ ) proportion of $\mathrm{IgG}_{4}$ in normal $\mathrm{IgG}$. In addition, most antibodies from both groups bind to the $\mathrm{A} 2$ and/or $\mathrm{C} 2$ domains as revealed by immunological investigations (Hoyer and Scandella, 1994; Scandella et al., 1989).

Nevertheless, differences do exist, the most striking one being the pattern of inactivation of factor VIII by these inhibitors (Hoyer and Scandella, 1994). Most inhibitors in inherited haemophilia A are type I, in which there is linear inactivation when the logarithm of residual FVIII:C is plotted against plasma concentration. These antibodies completely destroy all FVIII:C when present in high concentration. Most inhibitors in patients with acquired haemophilia have type II properties, where there is non-linear and incomplete inactivation (Kasper, 1991; Pruthi and Nichols, 1999). Thus, these patients may have a high inhibitor titre but still maintain some FVIII:C in their plasma.

\section{In Vitro Kinetic Study of the Two Types of Factor VIII Inhibitors}

The presence of an inhibitor to factor VIII, particularly a high-titre inhibitor associated with an anamnestic response, greatly complicates treatment. Kinetic studies of factor VIII inhibitors include experiments to determine the time course of inhibition and to ascertain the inhibition by various concentrations of inhibitors. These studies may be of importance in understanding the mechanisms by which these inhibitors interfere with FVIII:C. Possibly such studies may lead to improvements in treatment. In the last few decades, 
some attention has been drawn to this particular basic biological research area of factor VIII inhibitors.

\subsection{Kinetic Features of Factor VIII Inhibitors}

The first publications concerned with examining the kinetics of factor VIII inhibitors were based on case reports of small groups of patients with haemophilia A or acquired haemophilia (Andersen and Troup, 1968; Biggs and Bidwell, 1959; Green, 1968; Kernoff, 1972; Leitner et al., 1963; Pool and Miller, 1972; Shapiro, 1967). In these studies, investigators found an increasing amount of FVIII:C was inactivated by incubation with increasing concentrations of inhibitor. Biggs and Bidwell (1959), found a linear decrease of FVIII:C during a two hour incubation with inhibitors from both inherited and acquired haemophilia patients. All other studies found a curvilinear decline of FVIII:C with inhibitors from acquired haemophilia patients (Andersen and Troup, 1968; Green, 1968; Kernoff, 1972; Leitner et al., 1963; Pool and Miller, 1972; Shapiro, 1967). However, no clear idea about the kinetic classification of these inhibitors was formed from these early publications.

A more extensive study of classification for factor VIII inhibitors was published in 1972 by Biggs et al. (1972 a and b). They described a number of ways in which type I and type II inhibitors could be differentiated from each other. This was mainly by study of the time course of factor VIII inactivation. The methodology and its interpretation is complex, and does not lead to an easy and standardised approach suitable for studies on a large number of patients. A simpler method which showed differences between the two types of inhibitors was used by Gawryl and Hoyer (1982). The criteria they used for the 
classification of factor VIII inhibitors was that type I inhibitors can neutralise all (more than 98\%) available FVIII:C at high concentrations of inhibitor in a manner consistent with second-order kinetics, resulting in a linear inactivation response, while type II inhibitors cannot neutralise all available FVIII:C even at high concentrations of antibody (Gawryl and Hoyer, 1982). This seems to be a better way to distinguish type I and type II inhibitors, as the cut-off value "98\%" provides a definitive classification.

Since the publications by Biggs et al. (1972 a and b), there has been no detailed study of methods for the classification of the two types of factor VIII inhibitors. Most of the subsequent publications have cited their kinetic criteria as generally accepted (Santagostino, 1995; Hoyer et al, 1984; Kasper, 1984). However, interpretations of their methodology are complex and inconsistent (Allain and Frommel, 1974; Green et al., 1999; Michiels et al., 1997; Peerlinck et al., 1997). These problems are further discussed in Chapter 3.

Other studies have used the time course studies, proposed by Biggs et al. (1972 a and b), to classify patients in a case-report format (Bovill et al., 1985; Gordon et al., 1993; Gordon et al., 1994). A type II inhibitor was reported in a mild haemophilia patient, in which there was incomplete inactivation of FVIII:C in vivo and in vitro (Bovill et al., 1985). A type I inhibitor in a severe haemophilia patient was identified by a linear decline of FVIII:C with time (Gordon et al., 1993). In another study, aiming to assess the therapeutic efficacy of continuous infusion of a factor VIII preparation for a severe haemorrhage episode, type I kinetics with a second-order reaction was seen in two patients with inherited haemophilia A, and one patient with acquired haemophilia. The saturation of the inhibitors was 
achieved after adding an excess of factor VIII concentrate to the inhibitor plasma in vitro during a time course study (Gordon et al., 1994). None of these studies evaluated the usefulness of the kinetics studies published by Biggs et al. (1972 a and b). Therefore there is a need for a detailed analysis of these methods.

\subsection{Different Patient Groups with Different Antibody Kinetic Types}

Despite the difficulties in developing a standardised approach to the classification of inhibitor kinetics, it has become generally accepted that inhibitors in patients with severe haemophilia A are type I inhibitors, but type II inhibitors have also been observed in this group (Gilles et al., 1997; Hoyer and Scandella, 1994; Pruthi and Nichols, 1999). Inhibitors in patients with mild, moderate or acquired haemophilia usually show type II kinetics, but type I inhibitors have also been described in such patients (Gilles et al., 1997; Hoyer and Scandella, 1994; Kasper, 1991; Pruthi and Nichols, 1999; Sallah, 1999).

It seems possible that the kinetics would have a bearing on treatment of the patients. It has been claimed that it may be useful to know if an inhibitor is type II, since there is always some measurable FVIII:C in the plasma with these inhibitors (Gawryl and Hoyer, 1982). It could be predicted that patients with type II inhibitors might respond well to infusions of high doses of factor VIII, while type I inhibitor patients would not (Gilles et al., 1997; Handin, 1998; Kasper, 1991; Mosher, 1996; Rizza and Biggs, 1973). Conceptually, this seems reasonable, but to our knowledge there is not any published work to define this.

The hypothesis that patients with type II inhibitors respond better to infusions of high dose factor VIII is worthy of future investigation. Studies of this nature would require a test 
procedure to differentiate type I and type II inhibitors, which was suitable for use in routine diagnostic laboratories. It is therefore useful to review the in vitro kinetic studies, and reexamine the previous complex tests with the aim of designing a procedure which can be used to differentiate these inhibitors in a routine laboratory. This may provide practical application to the classification of the two types of factor VIII inhibitors in haemophilia A.

\section{Methods to Detect Anti-Factor VIII Antibodies}

Proper management of haemophilia A patients with factor VIII inhibitors requires accurate laboratory evaluation. Clotting assays and mixing experiments have been developed and validated for the reproducible quantitation of factor VIII inhibitors. The most usual assays in routine laboratories are the New Oxford method (Rizza and Biggs, 1973) and the Bethesda method (Kasper et al., 1975), in which a plasma concentrate or a multidonor pooled normal plasma, respectively, is used as the source of factor VIII. In addition to these coagulation tests, some immunoassays have been used to detect anti-factor VIII antibodies which may or may not inhibit the coagulant function of factor VIII.

\subsection{Bethesda Assay to Quantitate Inhibitor Titre}

Confirmation and quantitation of a factor VIII inhibitor in a patient with haemophilia A is established in routine coagulation laboratories by performance of the Bethesda assay. This assay has been a consensus method for clinical evaluation of factor VIII inhibitors since its first description in 1975 (Kasper et al., 1975), and is a uniform method widely used in routine diagnostic laboratories. In this assay, the reaction mixture is incubated for two hours at $37^{\circ} \mathrm{C}$, because these inhibitors are not usually fast-acting and are temperaturedependent. The Nijmegen modification has since improved the specificity and reliability 
by preventing $\mathrm{pH}$ drift, which if uncontrolled, causes deterioration of factor VIII hence false positive results (Verbruggen et al., 1995; Giles et al., 1998; Ehrenforth et al., 1992). Briefly, test plasma containing factor VIII inhibitors, or factor VIII deficient plasma as a control, is incubated at $37^{\circ} \mathrm{C}$ for two hours with an equal volume of buffered pooled normal plasma. The residual FVIII:C in both the test and control samples is then determined by one-stage factor VIII assay. The inhibitor titre is expressed in terms of Bethesda units (BU), where a test sample resulting in a residual FVIII:C of 50\% of the control is considered to have one Bethesda unit of inhibitor per millilitre (BU/ml) (Goldsmith, 1993; Luddington and Jennings, 1992; Verbruggen et al., 1995; White, 1994). The limit of detection by this method is generally agreed to be $0.5 \mathrm{BU} / \mathrm{ml}$ of factor VIII inhibitor in plasma.

It should be recognised that there are limitations to the Bethesda assay in that it is restricted to analysis of antibodies directed against functional epitopes, and it is unable to detect noninhibitory antibodies which may significantly influence plasma clearance and survival times of infused factor VIII (Gilles et al., 1993; Dazzi et al., 1996; Regnault and Stoltz, 1994; White, 1994). Fulcher et al. (1985) originally reported one patient in their series with an antibody that recognised an epitope in the B domain of factor VIII, a region not necessary for procoagulant activity. In a study by Gilles et al. (1993), the majority of antifactor VIII antibodies detected by an immunoblotting assay did not interfere with its function. Antibodies recognising non-functional regions on factor VIII may be an explanation for the shortened factor VIII survival observed in some patients who do not have antibodies detected by the Bethesda assay. Thus the evaluation of anti-factor VIII antibodies solely by a functional method may not provide an accurate evaluation of the 
specific antibody response. Low-level inhibitory antibodies and antibody-factor VIII complexes may also not be detected in the Bethesda assay, but this is a theoretical problem without supporting evidence (White, 1994).

\subsection{ELISA Method to Detect Anti-Factor VIII Antibodies}

To improve the detection rate of antibodies, immunoassays, which measure both inhibitory and non-inhibitory antibodies based on their immunologic reaction with factor VIII molecules, have been proposed as alternative methods. These immunoassays include immuno-neutralisation, immunoprecipitation, immunoblotting analysis, and ELISA techniques, including a novel adoption, the so-called antigen-containing strips method (Fulcher et al., 1985; Nilsson et al., 1990; Sanchez-Cuenca et al., 1990; Scandella et al., 1998; Shurafa and Kithier, 1995).

ELISA is the abbreviation of Enzyme-Linked ImmunoSorbent Assays and can be used to measure antibody titre. The antigen of interest is directly adsorbed onto a solid substrate. A primary unlabelled antibody, derived from blood or any other body fluid, binds to the antigen on the solid substrate. A secondary antibody, to immunoglobulin of the species of the primary antibody, is conjugated to an appropriate enzyme. When the substrate is added, a colour change develops, the intensity of which is proportional to the amount of primary antibody bound to the antigen.

For detection of factor VIII antibodies in patients, the patient plasma is used as the primary antibody. Early studies on the ELISA method to detect factor VIII antibodies in haemophilia patients were described in 1990, with one study proposing this method to be 
simple, accurate and highly sensitive for immunological characterisation of factor VIII antibodies (Nilsson et al., 1990; Sanchez-Cuenca et al., 1990). Subsequent reports have demonstrated the use of highly purified factor VIII, which is adsorbed onto microtitre plates, with a peroxidase-conjugated goat anti-human IgG antibody for ELISA detection of factor VIII antibodies (Gautier et al., 1996; Mondorf et al., 1994). The results suggest that ELISA may be a suitable complementary test to the Bethesda assay for factor VIII antibody screening in haemophilia and non-haemophilia patients, although it cannot be used instead of the coagulation tests (Gautier et al., 1996; Mondorf et al., 1994; Regnault and Stoltz, 1994).

The possibility of measurement of both inhibitory and non-inhibitory antibodies by ELISA was suggested in a study in which all 18 patients with allo- or auto- antibodies to factor VIII (inhibitor activity from 1 to $5,500 \mathrm{BU} / \mathrm{ml}$ ) exhibited clear positive reactions, while none of the normal plasma results were positive (Regnault and Stoltz, 1994). The authors suggested that inhibitor activity as low as $0.1 \mathrm{BU} / \mathrm{ml}$ would be clearly detected by means of their test. In 1996, an Italian research group using recombinant factor VIII as the coating antigen showed a high prevalence (41\%) of non-inhibitory antibodies in a group of 23 haemophilia patients whose inhibitor titres were undetectable by Bethesda assay (Dazzi et al., 1996).

The same research group subsequently reported that $81 \%$ of patients with the intron 22 inversion were found to have factor VIII antibodies by ELISA, compared with only $35 \%$ for those without the inversion. This implied that the intron 22 gene inversion is a major predisposing factor for the development of factor VIII antibodies (Vianello et al., 1997). 
The inhibitor prevalence revealed by the Bethesda assay in this study was $25 \%$ and $17 \%$ for patients with and without the inversion, respectively (Vianello et al., 1997). Disruption within intron 22 of the factor VIII gene has been found to be the causative mutation in $50 \%$ of patients with severe inherited haemophilia $A$. In this mutation there is intrachromosomal rearrangement of the tip of the long arm of the X chromosome, with a region within intron 22 of the factor VIII gene (Lakich et al., 1993).

The presence of factor VIII inhibitors in severe haemophiliacs with this gene inversion has been extensively evaluated. This is because patients with such a major gene defect are thought most likely to see factor VIII as a "foreign" protein and therefore develop inhibitors, as there is little or no circulating factor VIII in their plasma. In addition, the intron 22 gene inversion is a common mutation which is readily detected. The prevalence of factor VIII inhibitors reported in these patients ranged from $0 \%(0 / 10$, Goodeve et al., $1994)$ to $81 \%$ (13/16, Vianello et al., 1997), including 25\% (5/20, Ljung, 1994), 36\% (13/36, Tizzano et al., 1994), 37.5\% (18/48, Tizzano et al., 1995) and 42.4\% (25/59, Schwaab et al., 1995) in six individual studies. In addition, results from an international consortium, combining data from 642 patients with the inversion, indicated that $20 \%$ of them developed factor VIII inhibitors (Antonarakis et al., 1995). In general, it is now strongly believed that there is a definite correlation between the intron 22 gene inversion and the presence of factor VIII inhibitors, with data confirming the prevalence of inhibitors in these patients higher than for haemophilia in general. However, in severe haemophilia A, the proportion of patients with inhibitors in the subgroup of intron 22 gene inversion should not be different from that in the subgroup of other major gene mutations, such as large deletions, and point mutations resulting in stop-codons or frame shifts. 


\section{Epitope Detection on Factor VIII Peptides}

Although the anti-factor VIII immune response is clearly polyclonal, inhibitors tend to cluster to a few regions (Gilles et al., 1997). Since the mid-80's, Fulcher, Scandella and co-workers have been making progress in localising factor VIII epitopes using a variety of immunological and molecular approaches (Hoyer, 1991).

The mature factor VIII molecule is a single-chain protein made up of 2332 amino acid residues, which consist of a series of domains arranged in the order A1-A2-B-A3-C1-C2. Specific cleavages at amino acid positions 372, 740 and 1689 of the mature protein yield an active heterotrimer of 54, 44 and $72 \mathrm{KD}$ subunits (Figure 1.3). There are a number of regions in factor VIII which act as epitopes for inhibitors. However, the A2 domain (44 $\mathrm{KD}$ fragment from the heavy chain) and the $\mathrm{C} 2$ domain (segment on the $72 \mathrm{KD}$ fragment of the light chain) of factor VIII contain the epitopes targeted by most $(90 \%)$ inhibitory antibodies (Lubin et al., 1994; Scandella et al., 1989; Scandella et al., 1992). More precisely, the majority of antibodies are directed towards a $17.3 \mathrm{KD}$ segment located at the carboxy-terminal end of the $\mathrm{C} 2$ domain and/or towards a $18.3 \mathrm{KD}$ segment located at the amino-terminal end of the A2 domain (Scandella et al., 1988). In addition, patients may have multiple antibodies against multiple sites on the factor VIII molecule (Hoyer, 1991; Scandella, 1995). (Figure 1.2)

Immunoblotting of thrombin-cleaved factor VIII was used as the first approach to localise epitopes for anti-factor VIII antibodies in inhibitor plasmas. In one study by Fulcher et al. (1985), antibodies bound to either the $72 \mathrm{KD}$ (light chain) or the $44 \mathrm{KD}$ (heavy chain), or both. Using immunoblotting of deletion polypeptides expressed in Escherichia Coli (E. 
Coli) (Wood et al., 1984), these epitopes were localised to amino acid residues 322-372 between the A1 and A2 domains, 373-541 at the amino terminus of the A2 domain, and 2173-2332 of the C2 domain.

Later epitope mapping studies have shown the binding site for inhibitors to be much smaller fragments of factor VIII, such as residues 484-508 in the A2 domain and residues 2303-2332 in the C2 domain (Healey, 1995; Scandella et al., 1995 a). In addition, there are some studies showing that inhibitory epitopes may localise to the A1 domain or to somewhere uncertain within the light chain, but outside the $\mathrm{C} 2$ domain, e.g., the $\mathrm{A} 3$ domain (Gilles et al., 1993; Lollar, 1997; Scandella, 1996; Scandella et al., 1989). Recently, residues 1804-1819 of the A3 domain were identified as an epitope region, which comprises a binding site for factors IX and IXa (Zhong et al., 1998). Methods used to perform epitope mapping include using thrombin-cleaved fragments of factor VIII, recombinant factor VIII segments, and various immunoassays such as immunoblotting, immunoprecipitation, ELISA and immunoneutralisation assays.

Work which has been performed to detect the epitopes in factor VIII has built up data about the regions of the factor VIII molecule involved in antibody binding. More studies need to be done to further localise the epitopes to smaller peptide fragments, i.e., 20 amino acids, for the purpose of possible therapeutic application in the future, namely, the neutralisation of the inhibitory function of factor VIII inhibitors by infusion of epitopecontaining peptides to bind to the inhibitors in vivo. 


\section{Aims of the Project}

There is increasing knowledge concerning the mechanisms of and sites of action of inhibitors of factor VIII. For instance, the kinetics of the inhibitors in severe inherited haemophilia A tend to be different from those in mild inherited haemophilia A and acquired haemophilia A. Major functional epitopes for these inhibitors have been described and the pattern of binding may also differ in the different patient groups of haemophilia A, such as inherited severe, inherited mild, and acquired. There is also evidence that antibodies may be directed against non-functional epitopes. There is little or no knowledge concerning whether the epitope sites are related to the type of mutation in inherited haemophilia. To further develop our ability to explore these aspects it was planned to study the kinetics of action of inhibitors in a group of patients with inherited and acquired haemophilia A. However, the methods described for the characterisation of the kinetics of factor VIII inhibitors are complex, and interpretations in the literature are contradictory. In addition we planned to explore the possibility that our patients with haemophilia A might have inhibitors that bind to non-functional sites on factor VIII. A further project was to develop methods to detect binding of inhibitors in our patients to the known functional epitopes on the factor VIII molecule.

Therefore the overall aims of this thesis were:

1. To evaluate the available methodology for characterising the kinetics of factor VIII inhibitors.

2. To characterise the kinetics of factor VIII inhibitors in a group of our patients.

3. To explore the possibility that there may be inhibitors that bind to non-functional epitopes. 
4. To detect inhibitor binding to epitopes in the $\mathrm{A} 2$ and $\mathrm{C} 2$ domains of factor VIII.

These aims are part of a broader long term strategy of our department which is to explore the relationships between the epitope sites for these inhibitors, the kinetics of their action, and the nature of the haemophilia. This includes the relationship to whether the haemophilia is inherited or acquired, and for inherited haemophilia, the sites and nature of the mutation. 


\section{CHAPTER TWO}

\section{MATERIALS AND METHODS}

\section{Patient and Control Samples}

\subsection{Patients}

Most of the patients, including patients with inherited haemophilia $\mathrm{A}$ and acquired haemophilia A, were from the South Australian Haemophilia Centre at the Royal Adelaide Hospital, and the Adelaide Women and Children's Hospital. Plasma from the remaining patients was kindly supplied by Dr. Alison Street from the Alfred Hospital in Melbourne, Victoria.

To ensure plasma factor VIII levels were at baseline, blood samples were usually collected at least seven days after the administration of any factor VIIII therapy. However, for children who receive prophylaxis therapy three times a week, samples were collected just prior to a dose of factor VIII. Most samples were taken within the last three years, while the earliest sample was obtained in 1988.

\subsection{Sample Preparation}

Blood samples were collected into $1 / 10$ th volume $109 \mathrm{mM}$ trisodium citrate anticoagulant. Plasma and cells were separated by centrifugation at $3,000 \mathrm{rpm}(2,000 \mathrm{~g})$ in a Beckman J$6 \mathrm{~B}$ centrifuge for 10 minutes at $15-20^{\circ} \mathrm{C}$. Plasma was transferred to into a clean tube and re-centrifuged for another 10 minutes at 3,000 rpm. The supernatant, free of platelet contamination, was then removed and stored at $-70^{\circ} \mathrm{C}$ for measurement of factor VIII parameters. Plasma samples were kept at $4^{\circ} \mathrm{C}$ during transport when applicable. 
Prior to inhibitor testing, plasma samples were heated at $56^{\circ} \mathrm{C}$ for 15 minutes to destroy FVIII:C which could interfere with the calculation of residual FVIII:C in the test incubation mixture (Lloyd et al., 1997). After this heat inactivation, samples were centrifuged 1-2 minutes in an Eppendorf tube in a microfuge at 13,000 rpm to remove any precipitated protein.

\subsection{Normal Plasma Used in ELISA Assay}

Blood samples from normal donors were kindly provided by the Red Cross Blood Collection Centre. Plasma preparation was the same as described above. These samples were used for the control group for the detection of factor VIII antibody by ELISA.

\section{Materials}

\subsection{Bethesda Assay and Modifications for Kinetic Studies}

\subsubsection{Commercial reagents}

Factor VIII Deficient Plasma: Factor VIII Deficient Plasma from Organon Teknika Corporation (Durham, USA): 10 vials/1.0 ml each; Product Number 38920; Lot Numbers $103963,161180,161201,110910,110928,110944,110945$.

Coagulation Reference Plasma: Coagulation Reference Plasma Normal from Realm Biomedical (Brisbane, Australia): 10 vials/1.0 ml each; Product Number CRP-N; Lot Numbers 4011, 4012, 4013. (Calibrated against World Health Organisation International Rederence Plasma.) 
Quality Control Plasma: Dade ${ }^{\circledR}$ Ci-Trol® Coagulation Control, Level 1 from Dade International Inc (Miami, USA): 10 or 20 vials/1.0 ml each; Product Number B4244-10; Lot Numbers 825,1013 . (Factor VIII values for mean and 2 SD (standard deviation) range were established in-house.)

APTT Reagent: Dade ${ }^{\circledR}$ Actin ${ }^{\circledR}$ FSL Activated PTT Reagent from Dade Behring (Marburg, Germany): 10 vials/10 ml each; Product Number B4219-2; Lot Numbers 156, 202A, 527308.

Factor VIII Concentrate: AHF (High Purity) from CSL Limited (Parkville, Australia): 250 IU/vial reconstituted in $10 \mathrm{ml}$ injection water; Product Number R 29767; Lot Number L31100185D.

\subsubsection{Buffers and solutions}

Owren's Buffer pH 7.35: $5.88 \mathrm{~g}$ sodium diethylbarbiturate and $7.34 \mathrm{~g}$ sodium chloride in 1 litre distilled water, adjusted $\mathrm{pH} 7.35$.

Calcium Chloride 25mM: AnalaR® calcium chloride solution 1M stock (BDH, Production Number 19046) diluted $1 / 40$ in sterile water.

Imidazole Solution 5.0M: 680mg imidazole salt in $2 \mathrm{ml}$ distilled water.

Hydrochloric Acid Solution $1 N(1 N \mathrm{HCl}): 32 \%$ stock $\mathrm{HCl}$ (8.6 Normal/Molar) diluted 1/8.6 in distilled water. 
Buffered, Pooled Normal Plasma (B-PN): To $1 \mathrm{ml}$ pooled normal plasma (Batch Number PN38), $20 \mu$ imidazole solution (5M) was added to bring the solution to $0.1 \mathrm{M}$ imidazole, and $\mathrm{pH}$ was adjusted to 7.4 with $1 \mathrm{~N} \mathrm{HCl}(28 \mu \mathrm{l}$ per ml plasma).

\subsubsection{Equipment}

MLA Electra 1000C (Medical Laboratory Automation, Inc., New York, USA) automatic coagulation analyser: used to perform one-stage FVIII:C assays according to the operator's manual. Sample cups and cuvettes were also supplied by MLA.

\subsection{IgG Purification from Plasma}

\subsubsection{Purification kit and concentration column}

The ImmunoPure $(\mathrm{B})$ IgG Purification Kit was purchased from Pierce Chemical Company (Rockford, USA); Product Number 44441; Lot Numbers 97041168, 98010765, 99012160A.

Two products from Amicon, Inc. (Beverley, USA) were used: centrifugal filter device Centriprep ${ }^{\circledR}$ YM-10 (for larger volume of concentration); Product Number 4304; Lot Number L8PM9235D, and centrifugal filter device Centricon® YM-10 (for small volume of concentration); Product Number 4205.

\subsubsection{Buffers}

The following buffers were used: 
1. ImmunoPure ${ }^{\circledR}(\mathrm{G})$ IgG Binding Buffer from Pierce Chemical Company (Rockford, USA), 3.75 litres; Product Number 21011AD; Lot Number 9811670.

2. ImmunoPure ${ }^{\circledR}$ IgG Elution Buffer from Pierce Chemical Company (Rockford, USA), 1 litre; Product Number 21004AD; Lot Number 98122362.

3. Phosphate Buffered Saline (PBS) $\mathrm{pH}$ 7.4: $8 \mathrm{~g} \mathrm{NaCl}, 0.2 \mathrm{~g} \mathrm{KCl}, 2.27 \mathrm{~g} \mathrm{Na}_{2} \mathrm{HPO}_{4}$ (anhydrous), and $0.2 \mathrm{~g} \mathrm{KH}_{2} \mathrm{PO}_{4}$ in 1 litre distilled water, adjusted to $\mathrm{pH} 7.4$.

4. PBS pH $7.4-0.1 \%$ Tween: $1 \mathrm{ml}$ Tween 20® (Sigma) in 1 litre PBS pH 7.4.

\subsubsection{Optical Density (OD) detection}

WPA (Cambridge, UK) Biotech Photometer UV1101 (Series Number 3052) was used to measure OD at $490 \mathrm{~nm}$.

\subsection{ELISA Method to Detect Factor VIII Antibody}

\subsubsection{Commercial reagents}

2.3.1.1 Factor VIII Source to Coat Microtitre Plate

Formulated recombinant factor VIII produced by Baxter Healthcare Corporation (Deerfield, USA) Recombinate ${ }^{\mathrm{TM}}$ : $500 \mathrm{IU} / \mathrm{vial}$ reconstituted in $10 \mathrm{ml}$ water for injection; Product Number R 49620; Lot Number 2938M551AB. (Purchased from CSL Limited.) 
Unformulated recombinant factor VIII, a generous gift from Baxter Healthcare Corporation: $0.21 \mathrm{mg} / \mathrm{ml}, 1400 \mathrm{IU} / \mathrm{ml}$ reconstituted in $2 \mathrm{ml}$ injection water; Lot Number 1A03L003.

2.3.1.2 Monoclonal Primary Antibody

(murine) Monoclonal Antibodies Against Human Factor VIII:C from American Diagnostica Inc. (Greenwich, USA); Product Number ESH 8; Lot Number 990521.

\subsubsection{Secondary Antibody}

Peroxidase-Conjugated Rabbit Anti-Mouse Immunoglobulins from DAKO A/S (Glostrup, Denmark); Product Number P 0260; Lot Number 016 (102).

Peroxidase-Conjugated Rabbit Anti-Human IgG, Specific for Gamma-Chains from DAKO A/S; Product Number P 0214; Lot Number 115 (202).

2.3.1.4 1,2-Phenylenediamine Dihydrochloride (OPD·2HCl, OPD) Solution OPD Tablets, 2 mg For ELISA from DAKO A/S; Product Number S 2045; Lot Number 108 (101). Each tablet containing $3.5 \mathrm{mg} \mathrm{OPD} \cdot 2 \mathrm{HCl}$ corresponding to $2 \mathrm{mg}$ OPD. 2

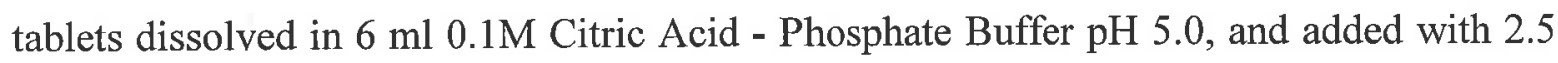
Hl 30\% Hydrogen Peroxide $\left(\mathrm{H}_{2} \mathrm{O}_{2}\right)$.

\subsubsection{Buffers}

Coating buffer (0.1M Carbonate Buffer $\mathrm{pH}$ 9.6): $2.93 \mathrm{~g} \mathrm{NaHCO}_{3}$ and $1.59 \mathrm{~g} \mathrm{Na}_{2} \mathrm{CO}_{3}$ (anhydrous) in 1 litre sterile water to dilute factor VIII. 
Washing buffer (PBS pH 8.0 - 0.1\% Tween): $8 \mathrm{~g} \mathrm{NaCl,} 0.2 \mathrm{~g} \mathrm{KCl}, 1.14 \mathrm{~g} \mathrm{Na} \mathrm{NaO}_{4}$ (anhydrous), and $0.2 \mathrm{~g} \mathrm{KH}_{2} \mathrm{PO}_{4}$ in 1 litre sterile water, adjusted to $\mathrm{pH} 8.0$, with $1 \mathrm{ml}$ Tween $20 ®($ Sigma) added.

Blocking buffer (PBS - Tween - 0.5\% Gelatin): 0.5g Gelatin (Sigma) added to $100 \mathrm{ml}$ PBS pH $8.0-0.1 \%$ Tween, dissolved at $37^{\circ} \mathrm{C}$.

$0.1 M$ Citric Acid - Phosphate Buffer pH 5.0: 1.46g Citric Acid. $\mathrm{H}_{2} \mathrm{O}$ and $2.374 \mathrm{~g}$ $\mathrm{Na}_{2} \mathrm{HPO}_{4} .2 \mathrm{H}_{2} \mathrm{O}$ in $200 \mathrm{ml}$ sterile water, adjusted $\mathrm{pH} 5.0$.

2.5 $\mathrm{M} \mathrm{H}_{2} \mathrm{SO}_{4}: 18 \mathrm{M}$ stock $\mathrm{H}_{2} \mathrm{SO}_{4}$, diluted $1 / 7$ in sterile water.

\subsubsection{Equipment}

Nunc A/S (Roskilde, Denmark) microtitre plates Nunc-Immuno ${ }^{\mathrm{TM}}$ Module C12 MaxiSorp ${ }^{\text {TM }}$ Surface; Product Number 473709; Lot Number 046654.

Dynatech Laboratories (Chantilly, USA) microplate reader MR 7000 (Series Number G0998) was used to read the OD at $490 \mathrm{~nm}$.

\subsection{Epitope Detection on Factor VIII Peptide}

\subsubsection{Recombinant DNA technology to produce factor VIII fragments}

\subsubsection{Commercial Products}

DNA polymerase with buffer: 
Roche Molecular System, Inc. (Branchburg, USA) AmpliTaq GoldTM (250U, 5U/ $\mu$ ), with GeneAmp ${ }^{\circledR}$ 10X PCR Buffer II \& $\mathrm{MgCl}_{2}$ Solution; Product Number N808-0241.

Boehringer Mannnheim (Alameda, USA) Expand High Fidelity PCR System (100U), with Expand HF buffer, 10Xconc. with $15 \mathrm{mM} \mathrm{MgCl}_{2}$; Product Number 1732641.

Polymerase chain reaction (PCR) products purification kit: Bresatec Pty. Ltd. (Thebarton, Australia) BRESAspin ${ }^{\mathrm{TM}}$ PCR Purification Kit (100); Product Number BT-2000-100.

Gel extraction kit: QIAGEN Pty. Ltd. (Clifton Hill, Australia) QIAquick Gel Extraction Kit (50); Product Number 28704.

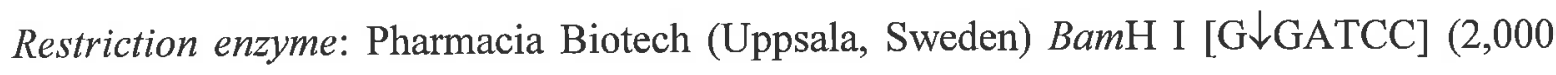
units); Production Number 27-0868-03.

Vector: Phamacia Biotech (Uppsala, Sweden) Glutathione S-transferase (GST) Gene Fusion Vector pGEX-2TK (25 $\mu$ g); Product Number 27-4587-01.

DNA ligation enzyme: New England BioLabs (Beverly, USA) T4 DNA Ligase 202S (20,000 units, 400,000 U/ml); Product Number 66.

DNA molecular weight marker: Bresatec Pty. Ltd. (Thebarton, Australia) SPP1/EcoRI

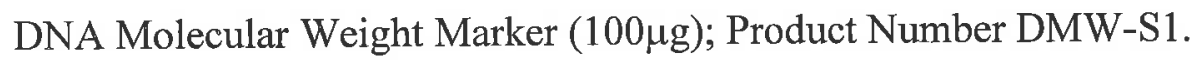


Isopropyl-beta-D-thiogalactoside (IPTG): Progen Industries Limited (Darra, Australia) IPTG (1g); Product Number 200-0120.

Protein purification resin: CLONTECH Laboratories, Inc. (Palo Alto, USA) TALON®) metal affinity resin (10 ml); Product Number 8901-1.

\subsubsection{Buffers}

5 X TBE buffer: $216 \mathrm{~g}$ Tris base 7-9 (Sigma), $110 \mathrm{~g}$ Boric Acid, and 80ml EDTA (0.5M, pH 8.0) in 4 litres of deionised water.

Colony cracking solution: $50 \mu \mathrm{l} \mathrm{NaOH}(1 \mathrm{M}), 50 \mu 1$ 10\% Sodium Dodecyl Sulphate (SDS), $10 \mu \mathrm{l}$ EDTA $(0.5 \mathrm{M}), 80 \mu \mathrm{l}$ Glycerol, $810 \mu \mathrm{l}$ sterile water, and the stain powder (bromophenol blue) to make $1 \mathrm{ml}$.

$4 X$ separating gel buffer: $37.4 \mathrm{~g}$ Tris base 7-9 (Sigma) and $8 \mathrm{ml} \mathrm{10 \%} \mathrm{SDS} \mathrm{in} 175 \mathrm{ml}$ sterile water, adjusted to $\mathrm{pH} 8.8$, then made up to $200 \mathrm{ml}$.

$2 X$ protein loading buffer: $2 \mathrm{ml}$ Tris- $\mathrm{HCl}(0.5 \mathrm{M}) \mathrm{pH} 6.8,1.6 \mathrm{ml}$ Glycerol, $3.2 \mathrm{ml} \mathrm{10 \%}$ SDS, $0.8 \mathrm{ml} 2-\beta$-mercaptoethanol, $0.4 \mathrm{ml} 0.05 \%$ bromophenol blue in sterile water to make $8 \mathrm{ml}$.

Lysis buffer: $2.5 \mathrm{ml} \mathrm{NaH} \mathrm{PO}_{4}(1 \mathrm{M}), 500 \mu \mathrm{l}$ Tris $(1 \mathrm{M}), 24 \mathrm{~g}$ Urea, and $5 \mathrm{ml} \mathrm{NaCl}(1 \mathrm{M})$ in 40 $\mathrm{ml}$ sterile water, adjusted to $\mathrm{pH} 8.0$, then made up to $50 \mathrm{ml}$. 
Protein elution buffer: $200 \mu \mathrm{l}$ Tris- $\mathrm{HCl}(1 \mathrm{M}) \mathrm{pH}$ 8.0, $1 \mathrm{ml} \mathrm{NaCl}(1 \mathrm{M}), 4.8 \mathrm{~g}$ Urea, $1 \mathrm{ml}$ Imidazole (2M), and $400 \mu 1 \mathrm{MES}$ (2-[N-morpholino] ethane sulfonic acid) (0.5M) in sterile water to make $10 \mathrm{ml}$, adjusted to $\mathrm{pH} 5.3$.

\subsubsection{PCR Equipment}

Perkin Elmer Corporation (Norwalk, USA) Perkin-Elmer Cetus DNA Thermal Cycler.

Perkin Elmer Corporation (Norwalk, USA) Perkin-Elmer DNA Thermal Cycler 480.

\subsubsection{Western blot with thrombin-cleaved factor VIII fragments}

\subsubsection{Thrombin Cleavage Reagents}

Factor VIII sources: Recombinate ${ }^{\mathrm{TM}}(50 \mathrm{IU} / \mathrm{ml})$ and Baxter unformulated factor VIII (1400 $\mathrm{IU} / \mathrm{ml}$ ) as previously described (see section 2.3.1.1).

Thrombin: Diagnostica Stago (Asnieres-sur-Seine, France) citrated calcium thrombin (human) STA® - Thrombin(2) (stock concentration $3 \mathrm{NIH}$ unit/ml); Product Number 00611 ; Lot Number 973512.

Reducing buffer: Pierce Chemical Company (Rockford, USA) ImmunoPure ${ }^{\circledR}$ Lane Marker Reducing Sample Buffer; Product Number 39000; Lot Number AD40446.

2.4.2.2 Protein Electrophoresis

7.5\% acrylamide gel made up of: 
Stacking Gel

Milli Q water

4 X Stacking buffer

4 X Separating buffer

$40 \%$ acrylamide

TEMED

0.1\% Ammonium Persulphate
$2.4 \mathrm{ml}$

$1 \mathrm{ml}$

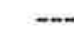

$540 \mu 1$

$11.3 \mu 1$

$63 \mu 1$
Separating Gel

$10.6 \mathrm{ml}$

$5 \mathrm{ml}$

$4 \mathrm{ml}$

$20 \mu 1$

$200 \mu 1$

ł: N'N'N'N'-Tetramethylethylenediamine, ultra pure grade (Amresco).

Protein lane marker: Life Technologies, Inc. (Gaithersburg, USA) BenchMark ${ }^{\text {TM }}$ Prestained Protein Ladder; Product Number 10748-010; Lot Number 1030096. Protein loading buffer: ImmunoPure ${ }^{\circledR}$ Lane Marker Reducing Sample Buffer as described before (section 2.4.2.1).

$10 X$ SDS-PAGE (polyacrylamide gel electrophoresis) running buffer: $30 \mathrm{~g}$ Tris base 7-9 (Sigma), 144g Glycine, and 10g SDS in 1 litre distilled water.

\subsubsection{Membrane Transfer}

Semi-Dry transfer buffer: $2.9 \mathrm{~g}$ Tris base 7-9 (Sigma), $1.45 \mathrm{~g}$ Glycine dissolved in $\sim 350 \mathrm{ml}$ distilled water, add $100 \mathrm{ml}$ methanol and $1.85 \mathrm{ml} 10 \% \mathrm{SDS}$, and make up to $500 \mathrm{ml}$.

Nitrocellulose membrane: Advantec MFS, Inc (Pleasanton, USA) Membrane Filters Cellulose Nitrate; Product Number A045A330R; Lot Number B27KD. 
Semi-Dry transfer unit: Hoefer Pharmacia Biotech Inc (San Francisco, USA) Semiphor ${ }^{\mathrm{TM}}$ Transphor; Hoefer ${ }^{\circledR}$ Number TE 70; Product Number 80-6210-34; Series Number 20039555.

\subsubsection{Blotting and Probing}

Blocking buffer : 5\% Skim Milk in PBS pH 7.4-0.1\% Tween.

Antibody dilution buffer: $2.5 \%$ Skim Milk in PBS pH $7.4-0.1 \%$ Tween.

Primary antibodies: Harlan Sera-Lab Limited (Loughborough, England) MAS 530 Monoclonal mouse anti-human factor VIII heavy chain; Product Number MAS 530p; Lot Number PC96L530. Harlan Sera-Lab MAS 531 - Monoclonal mouse anti-human factor VIII heavy chain; Product Number MAS 531p; Lot Number P92C531-2. ESH 8 (American Diagnostica) as described before.

Secondary antibodies: Amersham Pharmacia Bitotech UK Limited (Buckinghamshire, England) Anti-mouse IgG, peroxide-linked species specific $F\left(a b^{\prime}\right)_{2}$ fragment (from sheep); Product Number NA 9310; Lot Number 144832. Amersham Anti-human IgG, peroxidelinked species-specific whole antibody (from sheep); Product Number NA 933; Lot Number 148927.

Enhanced Chemiluminescence (ECL) detection reagents: Amersham Pharmacia Bitotech UK Limited (Buckinghamshire, England) ECL® Western blotting detection reagents; Product Number RPN 2109; Lot Number 61. 
ECL film: Amersham International plc. (Buckinghamshire, England) high performance chemiluminescence film Hyperfilm ${ }^{\text {TM }}$ ECL $^{\text {TM}}$; Product Number RPN 3103H; Lot Number 07020270215.

\section{Methods}

\subsection{In Vitro Kinetics of Factor VIII Inhibitors}

\subsubsection{Bethesda assay to quantitate FVIII:C inhibitors}

Factor VIII:C inhibitors were measured using the Nijmegen modification (Verbruggen $e t$ al., 1995) of the Bethesda method (Kasper et al., 1975). Haemophilia plasma (test) or factor VIII-deficient plasma (control) was incubated with an equal volume of buffered, pooled normal plasma ( $\mathrm{pH} 7.4)$ for two hours at $37^{\circ} \mathrm{C}$ in a closed plastic tube. Remaining FVIII:C (IU/ml) was measured by one-stage assay (Mannucci and Tripodi, 1992) and Residual FVIII:C (\%) expressed as a percentage of the control.

The strength of an inhibitor for a patient plasma is estimated from a graph, in which the log of residual FVIII:C (\%) is plotted against the Bethesda units on a linear scale. For the estimation to be valid, the percentage of residual FVIII:C must be in the linear region of the graph between $25 \%$ and $75 \%$ of control FVIII:C. If residual FVIII:C $>75 \%$, the inhibitor is reported as not detected. The test plasma must be further diluted and retested if residual FVIII:C is less than $25 \%$. The inhibitor titre for a test plasma is calculated as the Bethesda units from the graph multipled by the plasma dilution tested. An inhibitor with one Bethesda unit per $\mathrm{ml}$ is defined as the amount of antibody causing $50 \%$ reduction in FVIII:C compared to the control incubation. 
In this study, investigations of kinetics used variations of the basic method for the Bethesda assay. Modifications made included incubation time, antibody concentrations, and the initial factor VIII levels. In general, results were expressed as residual FVIII:C as a percentage of the control value, although the actual FVIII:C remaining is given in selected experiments.

\subsubsection{Time course studies}

Patient plasmas were incubated for two or four hours, in a small capped tube, with pooled normal plasma. Aliquots were removed at 0 minute, 15 -minute, and then at 30 -minute intervals until the end of the experiment. The amount of the remaining FVIII:C (IU/ml) at each time point was measured and the percentage of the control was calculated. The logarithm of residual FVIII:C (\%) was plotted against the incubation time in a semilogarithmic graph and was referred to as a "time curve" in this study.

\subsubsection{Antibody concentration studies}

A series of dilutions of patient plasma in factor VIII-deficient plasma was incubated with an equal volume of pooled normal plasma for two hours at $37^{\circ} \mathrm{C}$ and the remaining FVIII:C (IU/ml) was measured and the residual FVIII:C (\%) calculated. The logarithm of residual FVIII:C (\%) was plotted against the logarithm of antibody dilution, and was referred to as a "concentration curve" in this study. 


\subsubsection{Purification of IgG from patient plasma}

This was done to enable dilution curves to be determined in plasmas with low-titre inhibitors. Ten $\mathrm{ml}$ patient plasma was added to an immobilised protein $\mathrm{G}$ column and IgG was separated from other plasma components according to the manufacturer's instructions (ImmunoPure ${ }^{\circledR}$ IgG Purification Kit, Pierce). The eluents containing the greatest amounts of IgG (as shown by OD reading at $280 \mathrm{~nm}$ ) were then pooled and concentrated by centrifugation using centrifugal filter devices according to the manufacturer's instructions (Centriprep® 10 and Centricon ${ }^{\circledR} 10$, Amicon). The small volume (less than $500 \mu \mathrm{l}$ ) of concentrated IgG in buffer was used in antibody dilution studies to give a higher level of inhibitor titre.

\subsubsection{Effect of extra source of factor VIII}

Extended time courses were done to study the ability of bound antibodies to inhibit additional factor VIII (Bovill et al., 1985). Patient plasma was diluted in factor VIIIdeficient plasma and incubated with factor VIII concentrate (AHF) (approximately 1.0 $\mathrm{IU} / \mathrm{ml}$ in the mixture) for four hours, after which an additional amount of AHF was added to the mixture for another two or four hours incubation. The control mixture was made up of AHF with factor VIII-deficient plasma. Remaining FVIII:C (IU/ml) was measured for aliquots of the mixture every two hours, and plotted against the incubation time.

\subsubsection{Effect of the initial factor VIII concentration}

This experiment was designed to clarify the effect of the critical concentration ratio of antigen to antibody in the reaction mixture. A series of dilution curves of patient plasma with different initial factor VIII concentrations was studied. In addition to using undiluted 
pooled normal plasma in the incubation mixture, pooled normal plasma was diluted 2 in 3 , 1 in 2, and 1 in 4 in factor VIII-deficient plasma. Residual FVIII:C (\%) for each antibody dilution was plotted on a logarithmic scale against the initial factor VIII concentration (IU/ml).

\subsubsection{Mathematic analysis}

The results of the time course studies and antibody concentration studies were analysed using the statistical program GraphPad Prism version 3.00 for Windows 95 and NT, GraphPad Software, San Diego California USA, www.graphpad.com. Reaction models were chosen trying to distinguish the two different types of kinetic data in a mathematical way.

\subsection{ELISA Method to Detect Anti-Factor VIII Antibodies}

Microtitre plates Nunc-Immuno ${ }^{\mathrm{TM}}$ Module MaxiSorp ${ }^{\mathrm{TM}}$ were coated with $50 \mu 1 /$ well factor VIII (Recombinate ${ }^{\mathrm{TM}}$ or Baxter $\left.{ }^{\circledR}\right)$ unformulated factor VIII) in coating buffer, covered and incubated at $4^{\circ} \mathrm{C}$ overnight. After flooding the plates with washing buffer six times the next day, uncoated sites were blocked by adding plates with $100 \mu 1 /$ well of blocking buffer for 90 minutes at room temperature. Plates were washed again and primary antibody, diluted in blocking buffer, was added $(50 \mu \mathrm{l} / \mathrm{ml})$, then incubated at room temperature for two hours. A series of appropriate antibody dilutions were used, both for the murine monoclonal antibody ESH 8 and the plasmas from normal blood donors and haemophilia patients. After washing, the secondary antibody which was either rabbit anti-mouse or rabbit anti-human IgG with HRP (horse radish peroxidase) was incubated for one hour (50 $\mu 1 /$ well, $1 / 4000$ dilution of stock in blocking buffer, representing a saturating 
concentration). Substrate (OPD solution) for the HRP-linked enzyme was then added and the colour reaction allowed to process for 10 minutes before $\mathrm{H}_{2} \mathrm{SO}_{4}(50 \mu 1 /$ well $)$ was added to stop it. OD readings (at wavelength of $490 \mathrm{~nm}$ ) were made after five minutes by Dynatech microplate reader MR 7000.

\subsection{Epitope Detection on Factor VIII Peptide}

\subsubsection{Recombinant DNA technology using bacterial expression system to produce factor}

\section{VIII fragments}

The following was the procedure used in attempts to produce recombinant A1 fragment. Attempts to produce heavy chain, A1/A2, and A2 fragments were also performed during the research period of this project. However, none of them had achieved any successful result, despite the large amount of work and time spent. This suggests that there is still a lot to be done on the methodology development. This work was performed in conjunction with a current research project carried out in the Molecular Pathology Division at IMVS. The method and results following show parts of the work I undertook. The principle used to produce the A1 domain can be applied to other segments of factor VIII.

\subsubsection{PCR to Make Target Gene}

The cDNA (copy DNA) corresponding to the region encoding the factor VIII A1 domain was amplified by a PCR, with primers designed, to insert the BamH1 restriction enzyme site at both the 3'- and 5'- ends of the oligonucleotide chain. The A1 target gene starts from the H172 (heavy chain No. 172 base pair) and finishes at H1375 (heavy chain No. 1375 base pair) and the length of gene is $\sim 1.3 \mathrm{~kb}$. The base pair sequence recognised by Bam $\mathrm{H} \mathrm{I}$

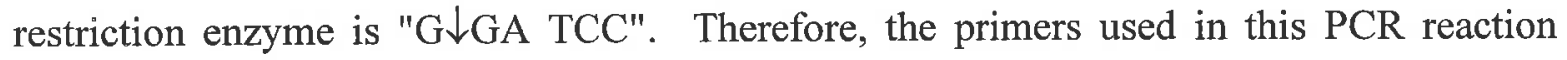


were designed to be as follows: the 5' primer (H172 BamH I, forwards) GAT GGA TCC ATG CAA ATA GAG CTC TCC ACC; and the 3' primer (H1375 BamH I, backwards) GCG GGA TCC CCA AGT TTT AGG ATG CTT CTT. The primers were purified and pre-checked with AmpliTaq Gold ${ }^{\mathrm{TM}}$ (Roche) PCR system for their working viability.

Expand $^{\mathrm{TM}}$ High Fidelity PCR System (Boehringer Mannheim) was used for the PCR reagents. The master mix was:

$\begin{array}{lr}10 \text { X buffer (with } \mathrm{MgCl}_{2} \text { ) } & 30 \mu \mathrm{l} \\ \text { Expand } & 4.2 \mu \mathrm{l} \\ \text { dNTPs } & 6 \mu l y m e r a s e \\ \text { H172 BamH I } & 12 \mu \mathrm{l} \\ \text { H1375 BamH I } & 12 \mu \mathrm{l} \\ \text { Milli Q } \mathrm{H}_{2} \mathrm{O} & 229.8 \mu \mathrm{l}\end{array}$

Each PCR reaction then contained $49 \mu \mathrm{l}$ of the above mixture plus $1 \mu \mathrm{l}$ of factor VIII cDNA, or $1 \mu 1 \mathrm{H}_{2} \mathrm{O}$ as non-DNA control. The reaction was performed in a DNA thermal cycler (Perkin-Elmer Cetus and Perkin-EImer), for 35 cycles with the thermal cycling conditions as follows:

$\begin{array}{lll}\text { Initial denaturation: } & 95^{\circ} \mathrm{C}, \quad 5 \text { minutes } & \\ \text { Denaturation: } & 95^{\circ} \mathrm{C}, 15 \text { seconds } & 35 \text { cycles } \\ \text { Annealing: } & 55^{\circ} \mathrm{C}, 30 \text { seconds } & \\ \text { Extension: } & 68^{\circ} \mathrm{C}, \quad 1 \text { minute } & \\ \text { Final extension: } & 72^{\circ} \mathrm{C}, 7 \text { minutes }\end{array}$

Following PCR, the products were visualised by electrophoresis on a $1 \%$ Agarose gel using standard techniques and photographed using a Polaroid camera system. 
BRESAspin ${ }^{\mathrm{TM}}$ PCR Purification Kit (Bresatec) was used to purify High Fidelity PCR products, according to the manufacturing handbook.

\subsubsection{Insertion, Transformation and Screening}

BamH I (Pharmacia Biotech) was used to digest both the target gene (purified A1) and the GST Gene Fusion Vector (pGEX-2TK, Pharmacia Biotech) at $37^{\circ} \mathrm{C}$. Ligation was then set up on ice using T4 DNA Ligase (BioLabs) and left at $4^{\circ} \mathrm{C}$ overnight. The ligation products were extracted the next day with phenol/chloroform. The pellet was finally washed in $70 \%$ ethanol and dried in Speed Vac, before it was resuspended in $10 \mu \mathrm{l}$ sterile water.

The vector with $\mathrm{A} 1$ inserted was transferred into the $E$. Coli competent cell, DH5 $\alpha$, subjected to electroporation, and the cells incubated on LB-Amp (Luria-Bertani Ampicilline) agar plates at $37^{\circ} \mathrm{C}$ overnight. Randomly selected colonies were removed from the agar plates. Colony cracking was carried out by adding cracking solution (lysis buffer plus loading buffer). Electrophoresis of each of these colony mixtures was used to reveal the expected molecular weight of the vector plus A1 DNA $(4.8+1.3 \mathrm{~kb})$.

The colonies identified from the electrophoresis were then allowed to grow in a bigger LBAmp culture at $37^{\circ} \mathrm{C}$ overnight. After mini-preparation of the culture and phenol/chloroform extraction, the vector DNA together with the inserted A1 from the $E$. Coli cells were purified, ready for the second round of gene insertion. BamH I digestion, ligation, and transformation were performed again, using this biologically-obtained target gene A1 fragment, rather than the PCR product A1. Colonies selected by colony cracking this time were then put into a large culture for protein expression. 


\subsubsection{Induction and Purification of the Proteins}

IPTG was used to induce the expression of the GST fusion proteins. Cells were grown at $30^{\circ} \mathrm{C}$, with $500 \mu \mathrm{l}$ of the culture taken out at the time points of $0 \mathrm{~h}, 0.5 \mathrm{~h}, 1 \mathrm{~h}, 2 \mathrm{~h}, 3 \mathrm{~h}$, and $4 \mathrm{~h}$ after the addition of IPTG $(0.1 \mathrm{mM})$ into the culture. Samples were spun, pellets resuspended in protein loading buffer, subjected to electrophoresis in $10 \%$ acrylamide gel and the gel stained with Coomassie blue. If the induction was successful, there should be extra bands at the molecular weight of the A1 peptide (about $47 \mathrm{KD}$ ) seen in the lanes after induction.

Purification of the target protein expressed by the $E$. Coli cells could be done by sonicating the pellet spun down from the cell culture, and washing the supernatant in sepharose beads. Purification was also tried by running the supernatant from sonication through the resin column with lysis buffer, and then washing the protein off with elution buffer.

\subsubsection{Western blot with thrombin-cleaved factor VIII fragment}

\subsubsection{Thrombin Cleavage and Protein Electrophoresis}

Recombinate $^{\mathrm{TM}}(50 \mathrm{IU} / \mathrm{ml})$ or Baxter unformulated factor VIII $(1400 \mathrm{IU} / \mathrm{ml})$, and STA®Thrombin(2) (3 NIH unit/ml) were used with the appropriate volumes so to keep the ratio of the amount of factor VIII:thrombin in the mixture about 100:1. Sterile water instead of thrombin was used as a negative control, and the cleavage time was 15 minutes. At the end of the reaction, ImmunoPure $($ reducing buffer was added as a stop reagent as well as the protein loading buffer. 
Proteins were heated at $95^{\circ} \mathrm{C}$ with loading buffer for $3-5$ minutes, cooled to room temperature and then loaded onto $7.5 \%$ acrylamide gel. The amount of factor VIII loaded in each lane was 1.5 IU. BenchMark ${ }^{\mathrm{TM}}$ protein marker was loaded $(10 \mu 1)$. The gel was run at $20 \mathrm{~mA}$ in $1 \mathrm{X}$ SDS-PAGE running buffer for 1.5 hours.

\subsubsection{Transfer to Membrane}

Following electrophoresis, the separating gel, a piece of nitrocellulose membrane of the same size, together with four pieces of blotting paper were soaked in Semi-Dry transfer buffer for approximately 10 minutes. Then assembled the gel-membrane sandwich in the Hoefer Semi-Dry transfer apparatus according to the instruction on the lid, and the transfer carried out at $0.8 \mathrm{~mA} / \mathrm{cm}^{2}$ for 90 minutes. After the transfer, the membrane was blocked in $5 \%$ milk in PBS- $0.1 \%$ Tween at $4^{\circ} \mathrm{C}$ for overnight.

\subsubsection{Incubation with Antibodies}

Primary antibodies, including monoclonal antibody MAS 530 (anti-heavy chain), MAS 531 (anti-light chain), and patient IgG were diluted $1 / 100$ in dilution buffer $(2.5 \%$ milk in PBS-0.1\%Tween). The membranes were cut into strips according to the design of the experiment and each was incubated with a different primary antibody at room temperature for two hours on a rocker. The membrane was washed once for 10 minutes, then $3 \times 5$ minutes with PBS-0.1\%Tween.

Secondary antibodies, including sheep anti-mouse and sheep anti-human IgG (Amersham) were diluted $1 / 2000$ and $1 / 1000$ separately in antibody dilution buffer. The corresponding secondary antibody was incubated with the membrane strip of the appropriate primary 
antibody for one hour at room temperature on a rocker. The wash steps were the same as for the primary antibody, with an extra rinse with PBS at the end.

\subsubsection{ECL Detection and Exposure to Film}

The ECL reagents were mixed 1:1 (1 ml of each) and placed in a glass plate. Excess buffer was blotted off the membrane, which was then placed face-down onto the glass plate. It was allowed one minute to incubate, while agitating gently. The membrane was then removed from the solution and any excess blotted off, before being wrapped in a piece of Glad-Wrap. The membrane was then placed into a cassette and films were developed after exposure for eight minutes. 


\section{CHAPTER THREE}

\section{IN VITRO KINETICS OF FACTOR VIII INHIBITORS}

\section{Introduction}

Circulating alloantibodies to factor VIII (factor VIII inhibitors) develop in patients with haemophilia A, and inhibit factor VIII coagulant activity (Ware et al., 1992). The development of inhibitors is particularly prevalent in patients with factor VIII $<5 \%$ of normal values (Kavanagh et al., 1981; McMillan et al., 1988). Factor VIII inhibitors also arise spontaneously as autoantibodies in patients without hereditary deficiency of factor VIII, causing the condition "acquired haemophilia" (Feinstain, 1993; Mosher, 1996).

The kinetic behaviour by which inhibitors inactivate FVIII:C activity is one of the features that has been occasionally used to characterise these inhibitors. Study of the kinetics of factor VIII inhibitors may provide a better understanding of the mechanisms by which inhibitors interfere with factor VIII:C activity. In addition, a knowledge of their kinetic behaviour may lead to improvements in clinical strategies for treatment. Biggs et al. were the first to describe the in vitro kinetic features of factor VIII inhibitors (Biggs et al., 1972). Their study provided criteria for the classification of factor VIII inhibitors based on kinetics, which have been widely accepted since the important publication in 1972 . Patients with severe inherited haemophilia tend to develop type I inhibitors, whereas patients with acquired haemophilia develop type II inhibitors (Gilles et al., 1997; Hoyer and Scandella, 1994; Pruthi and Nichols, 1999).

According to the description by Biggs et al., type I inhibitors exhibit second-order kinetics while type II inhibitors follow more complex kinetics. They examined kinetic behaviour 
mainly by studying the time course of inactivation of factor VIII by factor VIII inhibitors. They also examined the effect of varying the antibody concentration. Figure 3.1 shows three examples of time course studies taken from Biggs et al. (1972 a). The logarithm of the residual FVIII:C was plotted against the incubation time. There were two patterns observed, as claimed by Biggs et al. which were characteristic for type I inhibitors: a linear inactivation over two hour incubation (Figure 3.1 a), or a two-phase curved line (Figure 3.1 $\mathrm{b}$ and $\mathrm{c}$ ) ending with a stable residual FVIII:C level (or plateau) after two hour incubation (Figure $3.1 \mathrm{c}$ ), depending on different antibody concentrations used. In contrast, Figure 3.2 shows the time course for a type II inhibitor (Biggs et al., 1972 b). It was characterised by causing a rapid initial loss of FVIII:C followed by a slow gradual decline (Biggs et al., 1972 b).

While the study by Biggs et al. (1972) introduced many concepts and evidence for the kinetic features of the two types of factor VIII inhibitors, further descriptions of their kinetic behaviour have since been minimal. This likely reflects difficulties in applying the concepts of Biggs et al. to individual patient plasma samples. The problem is that the methods used in Biggs study were complex and do not clearly lead to a definitive protocol for the classification into the two types. This, for example, can be seen from the time course results in Figure 3.1 and Figure 3.2. The time course curves of type I kinetics in Figure 3.1 (c) show a pattern not distinguishable from the pattern in Figure 3.2 for type II kinetics. Furthermore, in the examples given in Figure 3.1, a linear time course was seen with one antibody 3.1 (a), and a curved time course was seen with the second antibody 3.1 (c). They both resulted in the same percentage of residual FVIII:C at the two hour incubation point, suggesting that the antibodies in the two cases were present in similar 


\section{FIGURE 3.1 Time Course Graphs for Type I Kinetics}

(Reprint from Biggs et al. The mode of action of antibodies which destroy factor VIII. I. Antibodies which have second-order concentration graphs. $\mathrm{Br}$ J Haematol 1972; 23: p. 128.) 

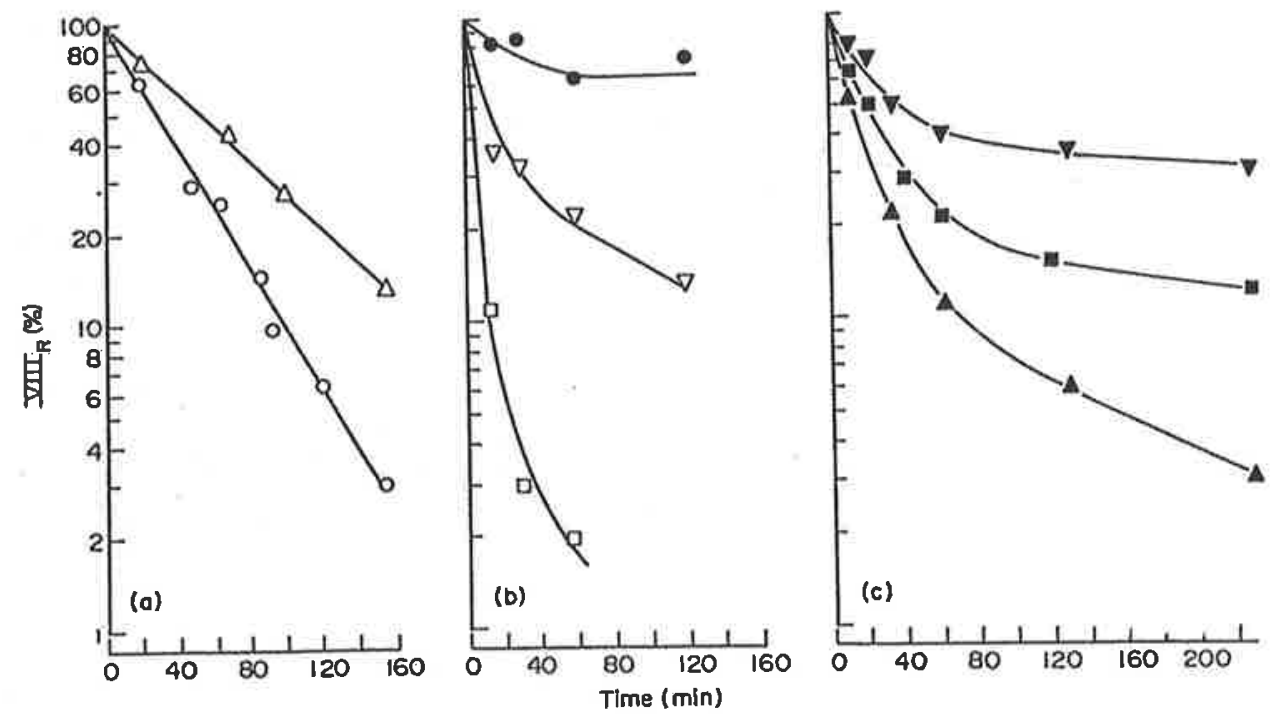

FIG I. Time-course graphs for antibodies $x, 2$ and 3. (a) Antibody $x$ tested at two concentrations: $I$ in $S(O)$ and $I$ in $10(\Delta)$. (b) Antibody 2 tested at three concentrations: undiluted $(\square), I$ in $I 0(\nabla)$ and $I$ in $100(O)$. (c) Antibody 3 tested at three concentrations: $X$ in $I 0(\Delta), X$ in $20(D)$ and $I$ in 30 $(\nabla)$. 


\section{FIGURE 3.2 Time Course Graph for Type II kinetics}

(Reprint from Biggs et al. The mode of action of antibodies which destroy factor VIII. II. Antibodies which give complex concentration graphs. Br J Haematol 1972; 23: p. 138.) 


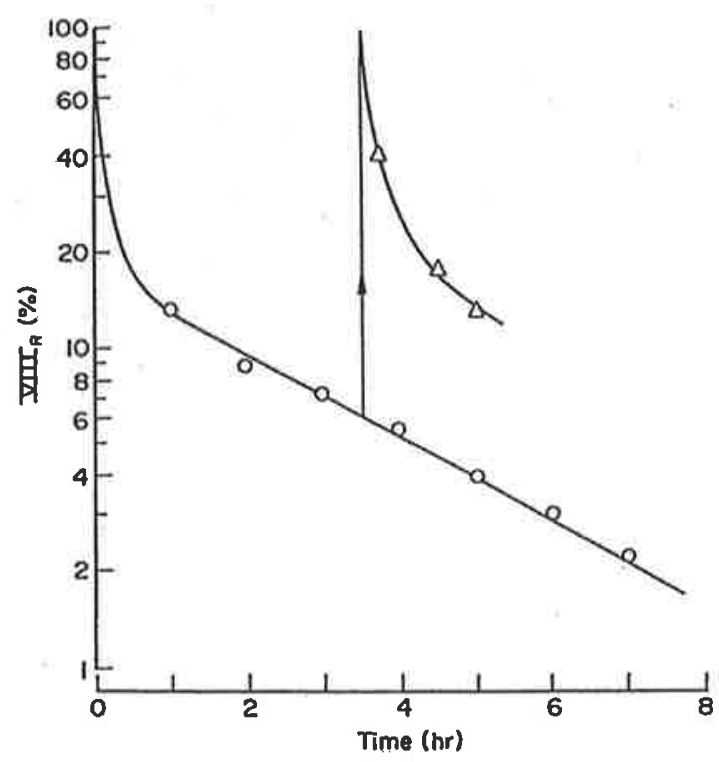

Frg 1. Time-course graphs for antibody s. After $3 \frac{1}{\mathrm{t}} \mathrm{hr}$ incubation more factor VIII was added and the disappearance of this factor VIII was also studied ( $\Delta$ ) (Kernoff, 1972). VIII $R=$ residual factor VIII. Initially added factor VIII (O). 
concentrations. Although Biggs et al. interpreted both of these as type I (second order) kinetics, they in fact appear quite different.

In 1982, Gawryl and Hoyer expressed the findings of Biggs et al. (1972 a and b) in a simplified form. They proposed that in an antibody concentration study, type I inhibitors at high antibody concentrations caused complete inactivation of FVIII:C, whereas type II inhibitors at the maximum concentration available were not able to completely inactivate FVIII:C (Gawryl and Hoyer, 1982). This property reflects the second-order kinetics of type I inhibitors and the more complex pattern of type II inhibitors.

Apart from these two major publications by Biggs et al. (1972 a and b), and Gawryl and Hoyer (1982), only nine further publications have been found, and the details of methodology are scant in these publications. Papers which give some detail include five using a study of time course (Allain and Frommel, 1974; Bovill et al., 1985; Gorden et al., 1993; Gorden et al., 1994; Green et al., 1999), and one using a study of antibody concentration (Peerlinck et al., 1997). Nevertheless, it is not clear from these papers how the methods adopted can provide true separation of the two types of factor VIII inhibitors. For example, in Green et al.'s study, there was only marginal statistical significance between the two types.

In addition, there are some publications that mention the kinetic classification of inhibitors but do not state the method used (Michiels et al., 1997; Peerlinck et al., 1993; Santagostino et al., 1995). Most of these publications were clinical case reports of only one to three patients (Bovill et al., 1985; Gorden et al., 1993; Gorden et al., 1994; Santagostino et al., 
1995). In some studies there were larger numbers of patients: four cases (one type I and three type II) in Michiels et al.'s work (1997); 12 cases (all type I) in Allain and Frommel's work (1974); and 14 cases (six type I and eight type II) in Green et al.'s work (1999).

From the above considerations, there are two main methods for determining the classification of a factor VIII inhibitor: study of the time course and study of the effect of varying the antibody concentration. An evaluation of the utility of these two different methods is required so that the approach to this problem can be standardised. It is therefore important to compare the application of both these methods to the same group of patients. Such a study has not been previously published.

Further justification for such a study is that, since the work of Biggs et al. was published, the methods to measure factor VIII:C have been automated and the one-stage assay has replaced the two-stage assay as the most common method. Factor VIII inhibitors are now generally measured in a system which quantitates their effect on FVIII:C in pooled normal plasma, rather than in factor VIII concentrate. Therefore, it is pertinent to review the study of in vitro kinetics of factor VIII inhibitors, with the aim of developing a standardised approach to classify inhibitor types in patient samples, using currently available methods to measure the effect of inhibitors on FVIII:C.

Therefore the aims of this study was to re-examine the previous methods used to classify the kinetic types of factor VIII inhibitors, and to develop a practical approach for a routine laboratory to classify inhibitor types based on in vitro kinetic behaviours. Specifically, the aims were: 
TABLE 3.1 Clinical Data of 11 Haemophilia A Patients in the Kinetic Study

\begin{tabular}{|c|c|c|c|c|c|c|c|c|c|c|}
\hline $\begin{array}{l}\text { Patient } \\
\text { Code }\end{array}$ & $\begin{array}{c}\text { Age } \\
\text { (years) }\end{array}$ & $\begin{array}{l}\text { Clinical } \\
\text { Severity }\end{array}$ & $\begin{array}{c}\text { Baseline } \\
\text { FVII:C } \\
\text { (IU/ml) }\end{array}$ & $\begin{array}{c}\text { Maximal } \\
\text { BU/ml } \\
\text { Recorded }\end{array}$ & $\begin{array}{l}\text { High/Low } \\
\text { Responder }\end{array}$ & $\begin{array}{l}\text { Age (year) } \\
\text { at Onset } \\
\text { of Inhibitor }\end{array}$ & $\begin{array}{c}\text { Baseline FVIII:C } \\
\text { with Inhibitor } \\
\text { Present (IU/ml) }\end{array}$ & $\begin{array}{c}\text { Treatment } \\
\text { Before } \\
\text { Inhibitor }\end{array}$ & $\begin{array}{c}\text { Treatment } \\
\text { After } \\
\text { Inhibitor }\end{array}$ & $\begin{array}{c}\text { Inhibitor Sample } \\
\text { Used in Study } \\
\text { (BU/ml) }\end{array}$ \\
\hline $1 . \mathrm{Du}$ & 27 & severe & $<0.01$ & 480 & high & child & $<0.01$ & $\overline{\mathrm{AHF}}$ & $\mathrm{A}, \mathrm{P}$ & 30 \\
\hline 2. Go & 29 & severe & 0.01 & 960 & high & child & 0.01 & AHF & A & 13 \\
\hline $3 . \mathrm{Hu}$ & 25 & severe & $<0.01$ & 134 & high & child & $<0.01$ & AHF & $A, N$ & 5 \\
\hline $4 . \mathrm{Fi}$ & 49 & severe & $<0.01$ & 6.5 & low & child & $<0.01$ & AHF & A & 3 \\
\hline $5 . \mathrm{Lo}$ & 52 & severe & $<0.01$ & 6.8 & low & child & $<0.01$ & AHF & $\mathrm{A}, \mathrm{P}$ & 1 \\
\hline $6 . \mathrm{EI}$ & 65 & mild & 0.12 & 1600 & high & 60 & 0.07 & $\mathrm{AHF}$ & $\mathrm{D}, \mathrm{A}$ & 700 \\
\hline $7 . \mathrm{Bo}$ & 27 & mild & 0.09 & 26 & high & 27 & $<0.01$ & AHF & Nil & 23 \\
\hline $8 . \mathrm{Ro}$ & 42 & mild & 0.04 & 4.2 & low & 26 & 0.04 & AHF & A & 2 \\
\hline 9. Si (M) & 69 & acquired & normal & 280 & high & 68 & 0.04 & -- & I, V & 140 \\
\hline 10. Bk (F) & 70 & acquired & normal & 33 & high & 70 & $<0.01$ & -- & I & 33 \\
\hline 11. Mi (F) & 27 & acquired & normal & 28 & high & 27 & 0.04 & - & I & 3 \\
\hline
\end{tabular}

High responder: those have a strong anamnestic response to human factor VIII, and the inhibitor titre increases to >10 BU/ml; Low responder: those have a weak response, and the inhibitor titre normally below $10 \mathrm{BU} / \mathrm{ml}$.

A: AHF (plasma derived factor VIII, high purity); P: porcine factor VIII; N: recombinant factor VIIa (Novoseven $($ )); D: desmopressin; I: immunosuppression therapy (e.g., prednisolone, azathioprine, cyclophosphamide, and vincristine); V: intravenous immunoglobulin (Intragam(8). None of the patients were treated with tolerisation therapy. 
1) To study the time course of action of factor VIII inhibitors.

2) To study the relationship between the degree of inhibition and the concentrations of inhibitors.

3) To determine the classification of the factor VIII inhibitors based on kinetics for a number of patients, and to determine whether in this group of patients, there is a relationship between the type of inhibitor and different clinical groups of patients (i.e., severe inherited, mild inherited and acquired).

4) To determine if a relationship exists between the kinetic classification and the epitope specificity of factor VIII inhibitors.

\section{Methods}

Eleven patients with haemophilia A and inhibitors, nine from South Australia (Royal Adelaide Hospital) and two from Victoria (The Alfred Hospital), were studied. This group comprised five patients with severe inherited haemophilia, three with mild inherited haemophilia and three with acquired haemophilia (one male and two females). A summary of the clinical and laboratory features of the 11 patients with haemophilia A used in this study is shown in Table 3.1. For two of the three acquired cases, there were no underlying conditions found (Case $9 \mathrm{Si}$ and Case $10 \mathrm{Bk}$ ), but they were elderly, which is a known risk factor (Pruthi and Nichols, 1999; Sallah, 1997). The third patient Mi (Case 11), was postpartum when the inhibitor was found. The cases with inherited haemophilia had all previously been treated with AHF (High Purity, CSL), which is the only human plasmaderived factor VIII concentrate available in Australia. None had been treated with recombinant factor VIII. The samples studied were all obtained at least seven days after the last infusion of factor VIII concentrate. Methods of plasma preparation and Bethesda 
assay to measure the inhibitor titre were as described before in Chapter 2 (sections 1.2 and 3.1.1). The inhibitor titre of the plasma samples used in the study ranged from 1.3 to 700 BU/ml. High-titre inhibitors (or high responders) were considered to be those with titres greater than $10 \mathrm{BU} / \mathrm{ml}$.

\section{Results}

\subsection{Effect of Incubation Time}

The time course of action of inhibitor plasma was studied during two to four hours in nine patients. In these experiments a dilution of patient plasma was incubated with pooled normal plasma as in the Bethesda method, and the residual FVIII:C level measured at time intervals up to two to four hours. The dilution of plasma was chosen such that the residual FVIII:C would be approximately $20-40 \%$ after two hours.

The results of two typical experiments (Case 1 and 9) are shown in Figure 3.3 and 3.4. Residual FVIII:C (\%) is shown plotted on both linear and logarithmic scales against the incubation time. The pattern of the time course graphs appeared to be the same for eight of the nine haemophilia patients studied. For each of them, there was a rapid initial decrease in FVIII:C to near maximal inactivation at about 30 minutes incubation, followed by a slight continuous decline. As expected, the degree of decrease in FVIII:C was greater at higher antibody concentration, as displayed with Case 1 in Figure 3.3, but the pattern seen with the two concentrations was similar. The majority of the graphs (eight of nine) therefore resembled the results seen in the studies of Biggs et al. (Figure $3.1 \mathrm{c}$ ). In Figure 3.5, the graph of the remaining patient sample (Case 11) shows a slow linear inactivation 


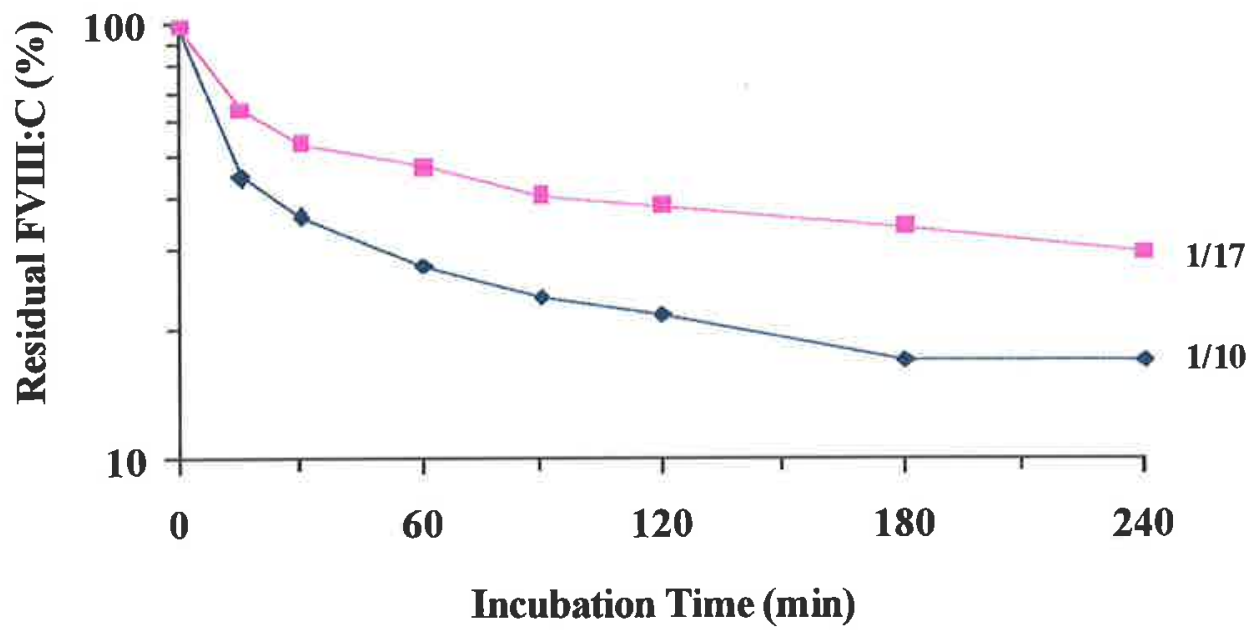

b

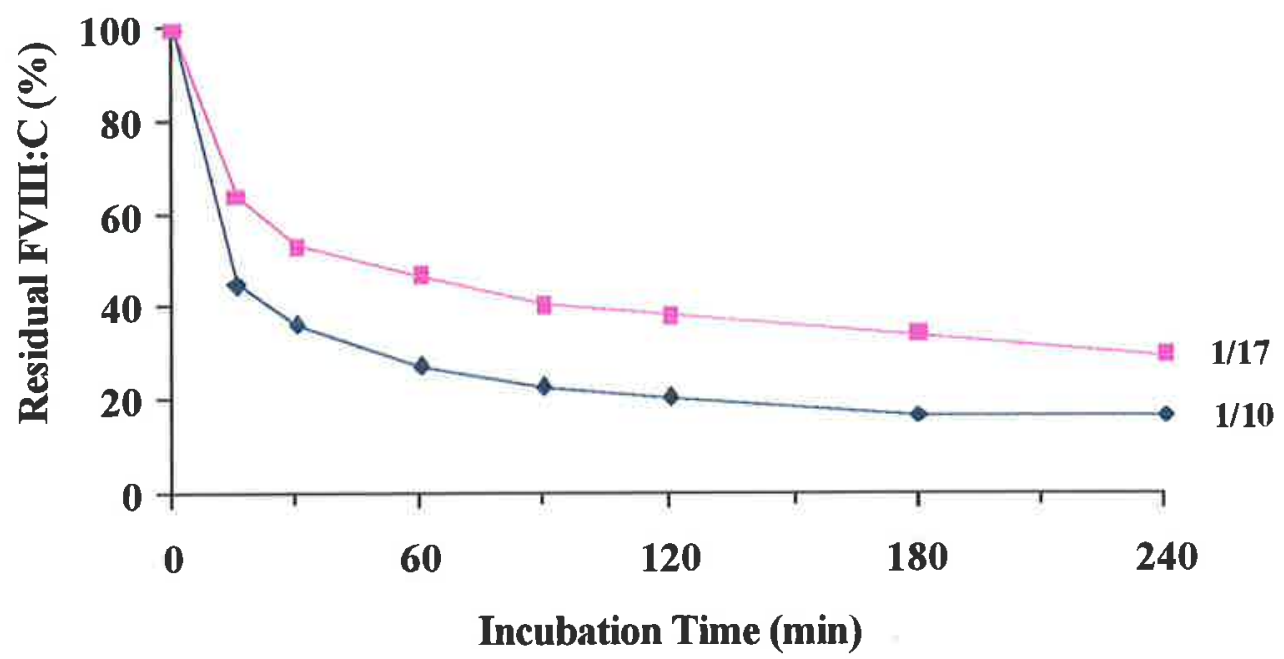

FIGURE 3.3 Effect of Incubation Time (Case 1, Du, BU=30)

A four hour incubation study was carried out using an equal volume of inhibitor plasma and pooled normal plasma at $37^{\circ} \mathrm{C}$. Residual FVIII:C (\%) was measured at multiple time points and plotted on both logarithmic (a) and linear (b) scales against the incubation time. Two dilutions of inhibitor plasma $(1 / 10$ and $1 / 17)$ were chosen to give approximately $20 \%$ to $40 \%$ of residual FVIII:C. 
$\mathbf{a}$

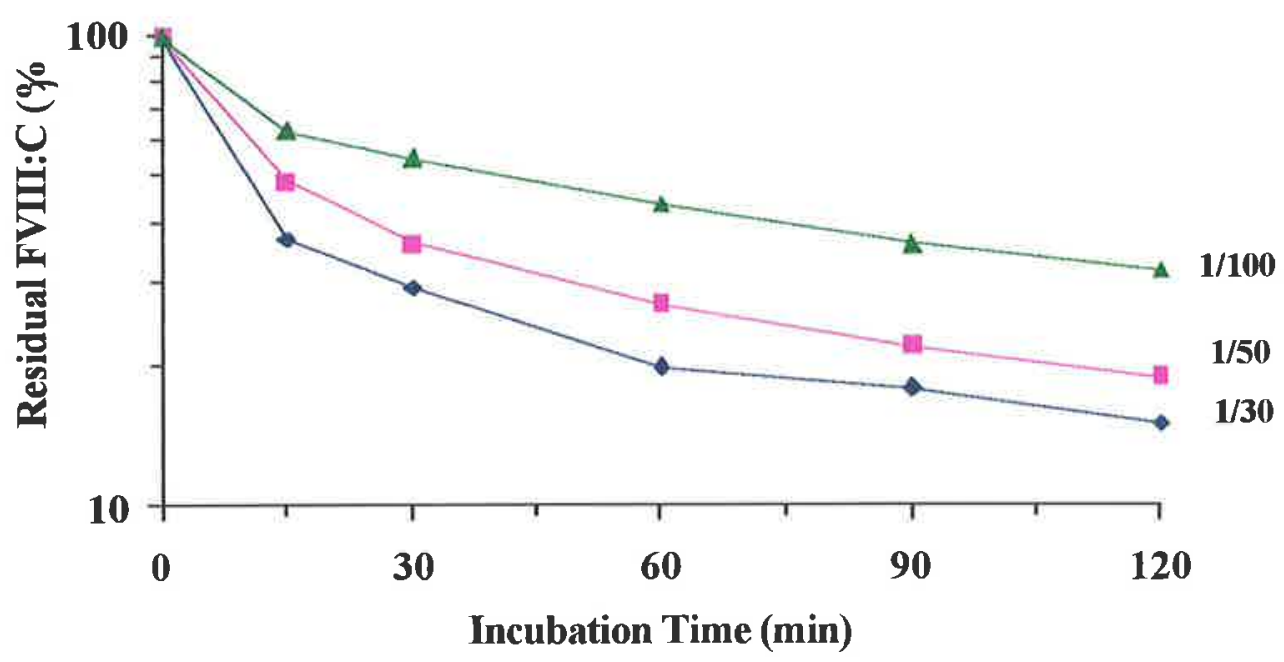

b

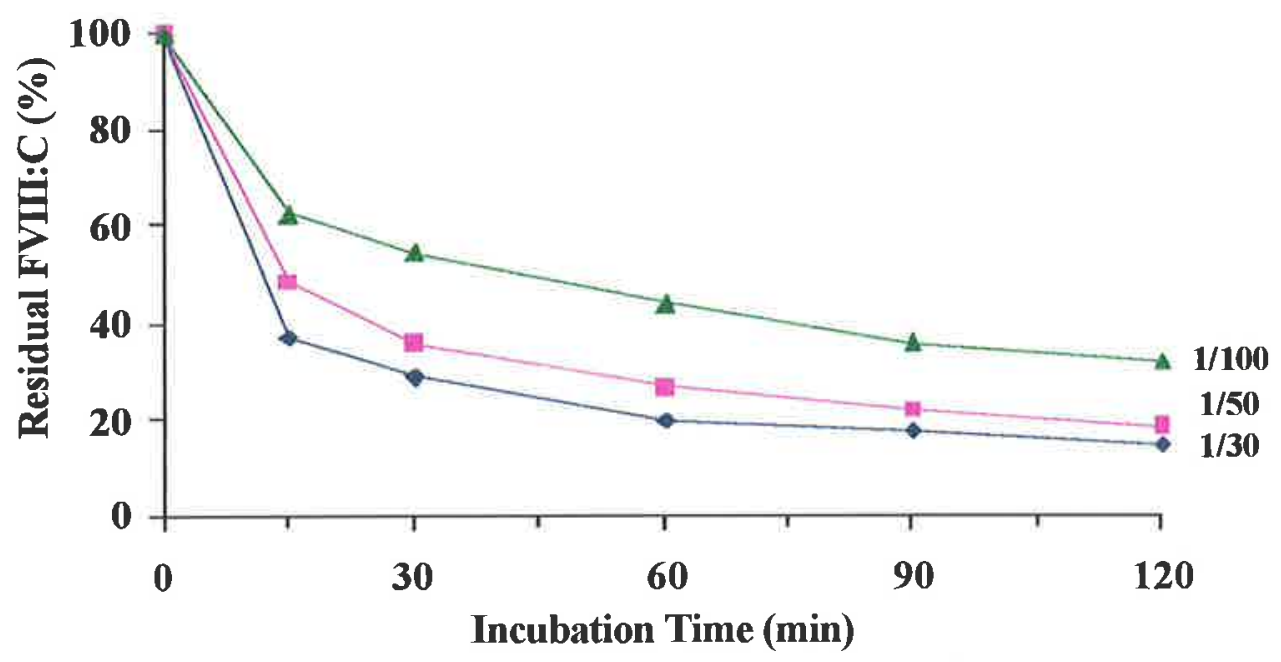

FIGURE 3.4 Effect of Incubation Time (Case 9, Si, BU=140)

A two hour incubation study was carried out using an equal volume of inhibitor plasma and pooled normal plasma at $37^{\circ} \mathrm{C}$. Residual FVIII:C (\%) was measured at multiple time points and plotted on both logarithmic (a) and linear (b) scales against the incubation time. Three dilutions of inhibitor plasma $(1 / 30,1 / 50$ and $1 / 100)$ were chosen to give approximately $20 \%$ to $40 \%$ of residual FVIII:C. 


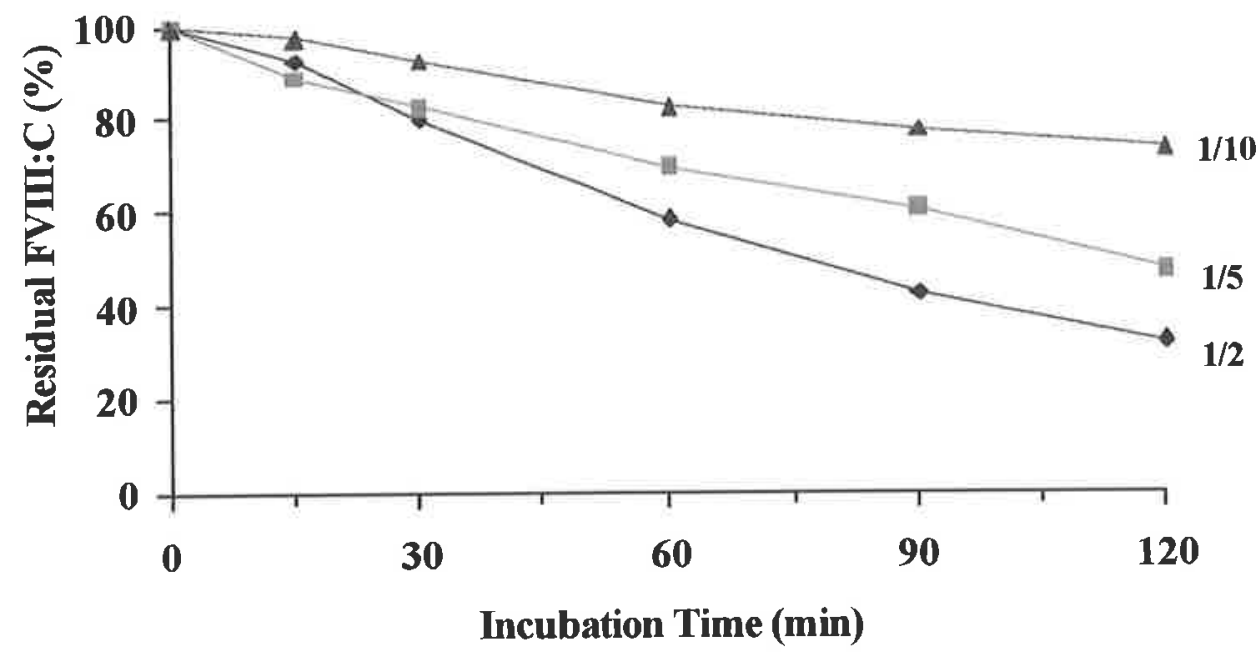

b

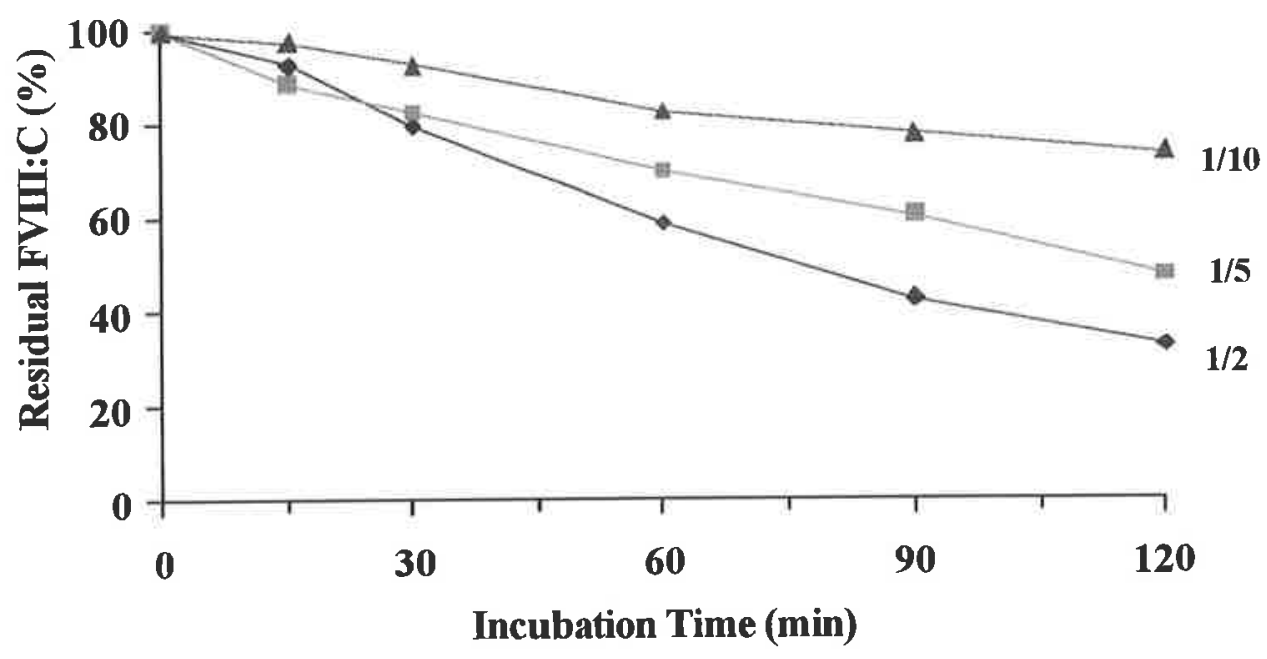

FIGURE 3.5 Effect of Incubation Time (Case 11, Mi, BU=3)

A two hour incubation study was carried out using equal volume of inhibitor plasma and pooled normal plasma at $37^{\circ} \mathrm{C}$. Residual FVIII:C (\%) was measured at multiple time points and plotted on both logarithmic (a) and linear (b) scales against the incubation time. Three dilutions of inhibitor plasma $(1 / 2,1 / 5$ and $1 / 10)$ were tested. 
over two hours. This is therefore the same as the pattern shown in the study by Biggs et al. (Figure 3.1 a).

Biggs criteria were interpreted by Allain and Frommel (1974) as follows: Type I kinetics is characterised by a plateau of residual FVIII:C occurring after a two hour incubation, whereas type II shows a gradual decline following a rapid initial drop. When our curves were analysed using these criteria all the curves showed a rapid decline, but none showed a convincing plateau. In all there is a slight further decline, even at four hours incubation. Green et al. (1997) interpreted type I kinetics to be characterised by a linear decline (second-order kinetics). However, in our data, only one (Case 11) appeared to be linear.

Because our patient population is diverse, containing patients with severe and mild inherited haemophilia as well as acquired haemophilia, we would have expected to find representatives of both type I and type II categories. Therefore, it seemed that in our patients the study of the time course did not discriminate between type I and type II kinetics. However, it was also possible that all the patients were of the same type, presumably type I.

To further analyse this, it was questioned whether there may be differences in the slope of the slow decline seen after the first phase that would allow a classification to be made. In order to investigate such a possibility, nonlinear regression with the equation for two phase exponential decay was performed using GraphPad Prism version 3.00 to fit the model to the data. The equation is as follows:

$$
Y=\operatorname{Span} 1 \cdot e^{-k_{1} \cdot x}+\operatorname{Span} 2 \cdot e^{-k_{2} \cdot x}+\text { Plateau }
$$


The $\mathrm{X}$ value is the incubation time, and the $\mathrm{Y}$ value is the residual FVIII:C in percentage. As defined in the program, phase 1 refers to the second (slow reaction) phase, while phase 2 refers to the first (fast reaction) phase. $K_{1}$ and $K_{2}$ are the rate constants in the second (slow) phase and first (fast) phase respectively. The greater the $\mathrm{K}_{1}$ value, the steeper the slope of the second inactivation phase. "Plateau" is the minimum residual FVIII:C (\%) that is automatically determined from the equation of the curve. "Span $1 "$ is the distance between the "Plateau" and the Y value at the inflextion point of the decay curve. "Span2" is the distance between the inflextion point to the maximal Y value $(100 \%$, i.e., the $\mathrm{Y}$ value when $\mathrm{X}$ value equals to zero).

The analysis showed that the decay curve for all patients was similar. Without exception, the model fitted well with each set of patient time course data. There was no significant difference in the values of the relevant computer-generated parameters. In particular, the $\mathrm{K}_{1}$ values were not significantly different from each other, as shown by the overlapping ranges for the $95 \%$ confidence intervals for $\mathrm{K}_{1}$. Table 3.2 gives the time course results analysed by the computer program. Case 11 (Mi) showed a residual FVIII:C (\%) which appeared to be linear rather than a curve by visual observation. The mathematical model did not fit her data, as well as shown by a very wide confidence interval for $\mathrm{K}_{1}$.

\subsection{Antibody Concentration Study}

\subsubsection{Visual observation of concentration graphs}

Experiments were carried out to ascertain the effects of varying the concentration of inhibitors on the inactivation of factor VIII. A series of dilutions of plasma samples, including full strength (FS) plasma, were incubated with pooled normal plasma for two 
TABLE 3.2 Comparison of Time Course Results from the Two Phase Exponential Decay Model Analysis with the Kinetic Classification by Antibody Concentration Study on 9 of 11 Patients

\begin{tabular}{|c|c|c|c|c|c|c|}
\hline \multirow{2}{*}{$\begin{array}{l}\text { Patient Code } \\
\text { (Plasma } \\
\text { Dilution) }\end{array}$} & \multicolumn{5}{|c|}{ Time Course Study (Two Phase Model Analysis) } & \multirow{2}{*}{$\begin{array}{l}\text { Antibody } \\
\text { Concentration } \\
\text { Study }\end{array}$} \\
\hline & $\begin{array}{l}\text { Data } \\
\text { Points }\end{array}$ & $\mathbf{R}^{2}$ & Plateau & $\mathbf{K}_{1}$ & $\begin{array}{l}95 \% \text { Confidence } \\
\text { Interval for } K_{1}\end{array}$ & \\
\hline $\begin{array}{r}\text { 1. } \mathrm{Du}(1 / 10) \\
\mathrm{Du}(1 / 17)\end{array}$ & $\begin{array}{l}8(4 h) \\
8(4 h)\end{array}$ & $\begin{array}{l}0.9997 \\
0.9992\end{array}$ & $\begin{array}{l}0.1628 \\
0.2512\end{array}$ & $\begin{array}{l}0.017 \\
0.008\end{array}$ & $\frac{0.010 \text { to } 0.023}{-0.001 \text { to } 0.016}$ & $\bar{I}$ \\
\hline $\begin{array}{r}\text { 2. Go (1/5) } \\
\text { Go }(1 / 8)\end{array}$ & \begin{tabular}{|l|}
$8(4 h)$ \\
$8(4 h)$
\end{tabular} & $\begin{array}{l}0.9992 \\
0.9980\end{array}$ & $\begin{array}{l}0.0858 \\
0.1026\end{array}$ & $\begin{array}{l}0.012 \\
0.005\end{array}$ & $\frac{0.004 \text { to } 0.021}{-0.004 \text { to } 0.014}$ & I \\
\hline $\begin{array}{r}3 . \mathrm{Hu}(1 / 2) \\
\mathrm{Hu}(1 / 4)\end{array}$ & \begin{tabular}{|l|}
$8(4 h)$ \\
$8(4 h)$
\end{tabular} & $\begin{array}{l}0.9996 \\
0.9986\end{array}$ & $\begin{array}{l}0.0518 \\
0.1916\end{array}$ & $\frac{0.010}{0.006}$ & $\begin{array}{c}0.002 \text { to } 0.018 \\
-0.004 \text { to } 0.015\end{array}$ & $\mathrm{I}$ \\
\hline $\begin{array}{r}\text { 4. } \mathrm{Fi}(5 / 6) \\
\mathrm{Fi}(3 / 5)\end{array}$ & \begin{tabular}{|l|}
$8(4 h)$ \\
$8(4 h)$
\end{tabular} & $\begin{array}{l}0.9996 \\
0.9998\end{array}$ & $\begin{array}{l}0.1217 \\
0.2725\end{array}$ & $\begin{array}{l}0.019 \\
0.014\end{array}$ & $\begin{array}{l}0.011 \text { to } 0.028 \\
0.000 \text { to } 0.029\end{array}$ & I \\
\hline $\begin{array}{r}\text { 5. Lo (FS) } \\
\text { Lo }(1 / 5)\end{array}$ & $\frac{6}{6}$ & $\begin{array}{l}0.9979 \\
0.9848\end{array}$ & $\begin{array}{l}0.2382 \\
0.7111\end{array}$ & $\begin{array}{l}0.012 \\
0.006\end{array}$ & $\frac{-0.142 \text { to } 0.166}{-0.345 \text { to } 0.356}$ & I \\
\hline $\begin{array}{r}6 . \mathrm{El}(1 / 300) \\
\mathrm{El}(1 / 500)\end{array}$ & $\frac{6}{6}$ & $\begin{array}{l}1.0000 \\
0.9993\end{array}$ & $\begin{array}{l}0.1599 \\
0.3319\end{array}$ & $\begin{array}{l}0.023 \\
0.022\end{array}$ & $\frac{0.023 \text { to } 0.023}{-0.110 \text { to } 0.155}$ & II \\
\hline 7. Bo (1/12) & 6 & 1.0000 & 0.1343 & 0.017 & -0.048 to 0.082 & I \\
\hline 8. Ro & - & - & - & - & - & I \\
\hline \begin{tabular}{|l} 
9. $\mathrm{Si}(1 / 30)$ \\
$\mathrm{Si}(1 / 50)$ \\
$\mathrm{Si}(1 / 100)$
\end{tabular} & $\begin{array}{l}\frac{6}{6} \\
6\end{array}$ & $\begin{array}{l}0.9997 \\
1.0000 \\
0.9999\end{array}$ & \begin{tabular}{|l|}
0.1339 \\
0.1444 \\
0.2369
\end{tabular} & $\begin{array}{l}0.029 \\
0.014 \\
0.015\end{array}$ & $\begin{array}{c}-0.131 \text { to } 0.189 \\
0.001 \text { to } 0.028 \\
-0.019 \text { to } 0.049\end{array}$ & II \\
\hline $10 . \mathrm{Bk}$ & - & - & - & - & - & I \\
\hline 11. $\mathrm{Mi}(1 / 10)$ & 6 & 0.9867 & 0.5287 & 0.007 & -735 to 735 & II \\
\hline
\end{tabular}

Time Course Study: Results generated by the computer analysis using GraphPad Prism statistical program showing: $\mathrm{R}^{2}$ as an indication of goodness of fit; The best-fit values for Plateau (minimum residual FVIII:C\%) and $\mathrm{K}_{1}$ (rate constant of slow phase); The $95 \%$ Confidence Interval of $K_{1}$. There is no significant difference between the $K_{1}$ values for the nine patient samples, and their $95 \%$ confidence intervals overlap with each other.

However, all these patient samples were classified as to have either type I or type II inhibitor by the later Antibody Concentration Study.

(4h): incubation duration for four hours instead of two. 
hours, and the residual FVIII:C was measured. On a dilution curve graph, for plasma samples with high-titre factor VIII inhibitor level $(>10 \mathrm{BU} / \mathrm{ml})$, residual FVIII:C plotted against various antibody dilutions showed two different patterns on logarithm scale (Figure 3.6 and 3.7). A steep slope and complete (>98\%) FVIII:C inactivation at high antibody concentrations was seen with two severe cases (Case 1, BU=30 and Case 4, BU=13) and one mild (Case 7, BU=23) case (Figure 3.6 and 3.7). This fits the definition of a type I inhibitor as given by Gawryl and Hoyer (1982). A curvilinear pattern with a FVIII:C plateau at high antibody concentrations was seen with one mild (Case $6, \mathrm{BU}=700$ ) and one acquired (Case 9, BU=140) cases (Figure 3.6 and 3.7). This indicated incomplete inactivation of FVIII:C even when antibody is in excess, and fits the definition of type II (Gawryl and Hoyer, 1982). The residual FVIII:C at full strength plasma for Case 6 and Case 9 were approximately $6 \%$ and $12 \%$ respectively. Thus, the difference between the two types of factor VIII inhibitors in their in vitro kinetic behaviour was clearly shown in an antibody concentration graph, and this is consistent with the demonstration in Gawryl and Hoyer's studies (Gawryl and Hoyer, 1982).

For the other six patient samples, it was not possible to characterise the concentration response because of the low concentration of inhibitors. It seemed that it might be possible to increase the titre of the inhibitor by concentrating it. One way to do this is to purify $\operatorname{IgG}$ from plasma, and then subject it to a concentration procedure.

\subsubsection{IgG purification for low-titre inhibitors}

In plasma samples with inhibitor level less than $10 \mathrm{BU} / \mathrm{ml}$, no clear classification could be made because the antibody concentration was not high enough to demonstrate whether or 


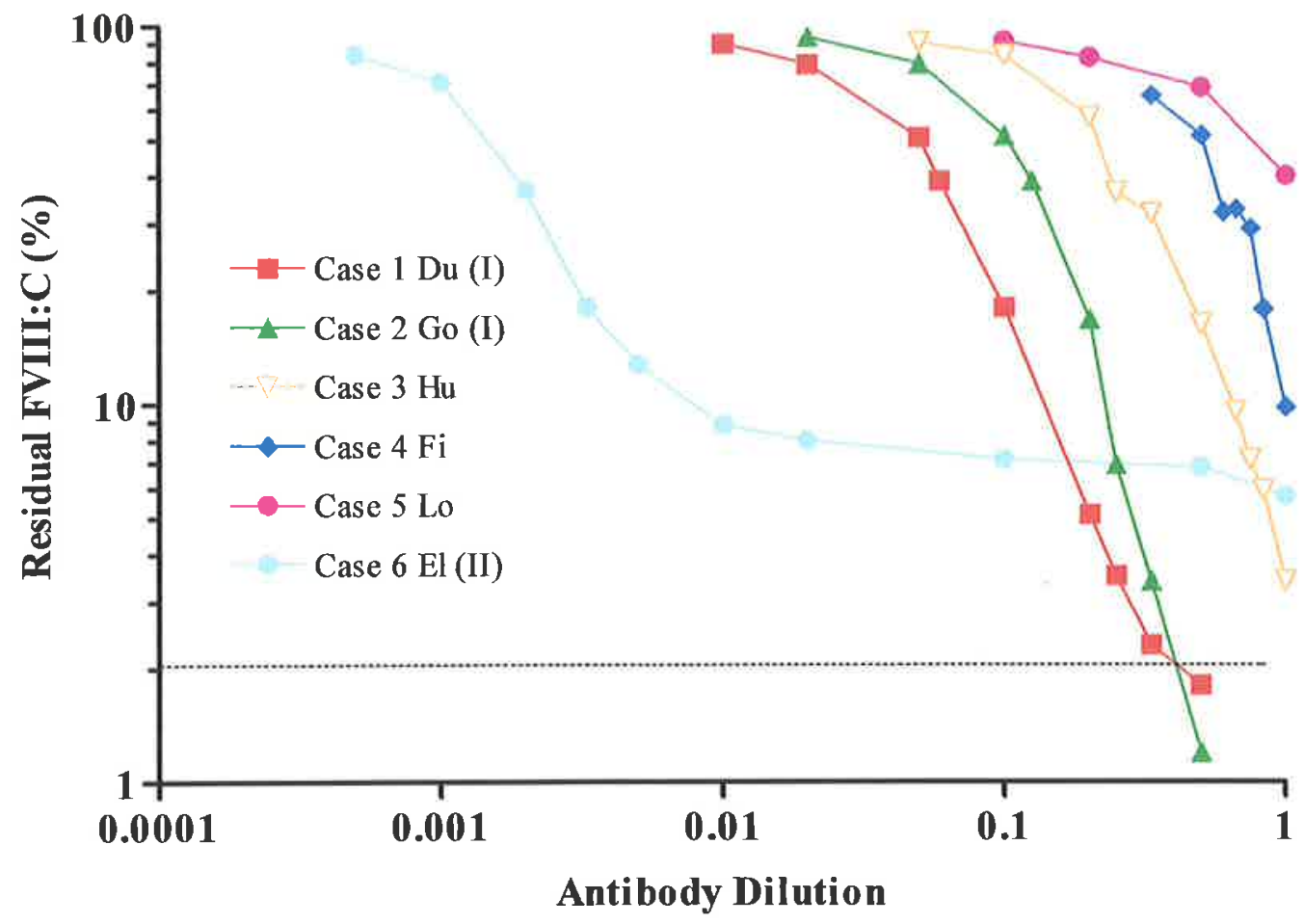

FIGURE 3.6 Effect of Antibody Concentration

(Cases 1-6)

A series of plasma dilutions for six cases were incubated for two hours with pooled normal plasma. Residual FVIII:C (\%) was plotted against antibody dilution, both on logarithmic scales. Case 1 and 2 were classified as having a type I inhibitor, while Case 6 was classified as having a type II inhibitor. Inhibitors from Case 3, 4 and 5 were unable to be definitely classified because of the low titre.

The dashed line indicates $2 \%$ residual FVIII: $\mathrm{C}$, which is the minimum detectable residual FVIII:C in the experiment (corresponds to FVIII:C level of $0.01 \mathrm{IU} / \mathrm{ml}$ ). 


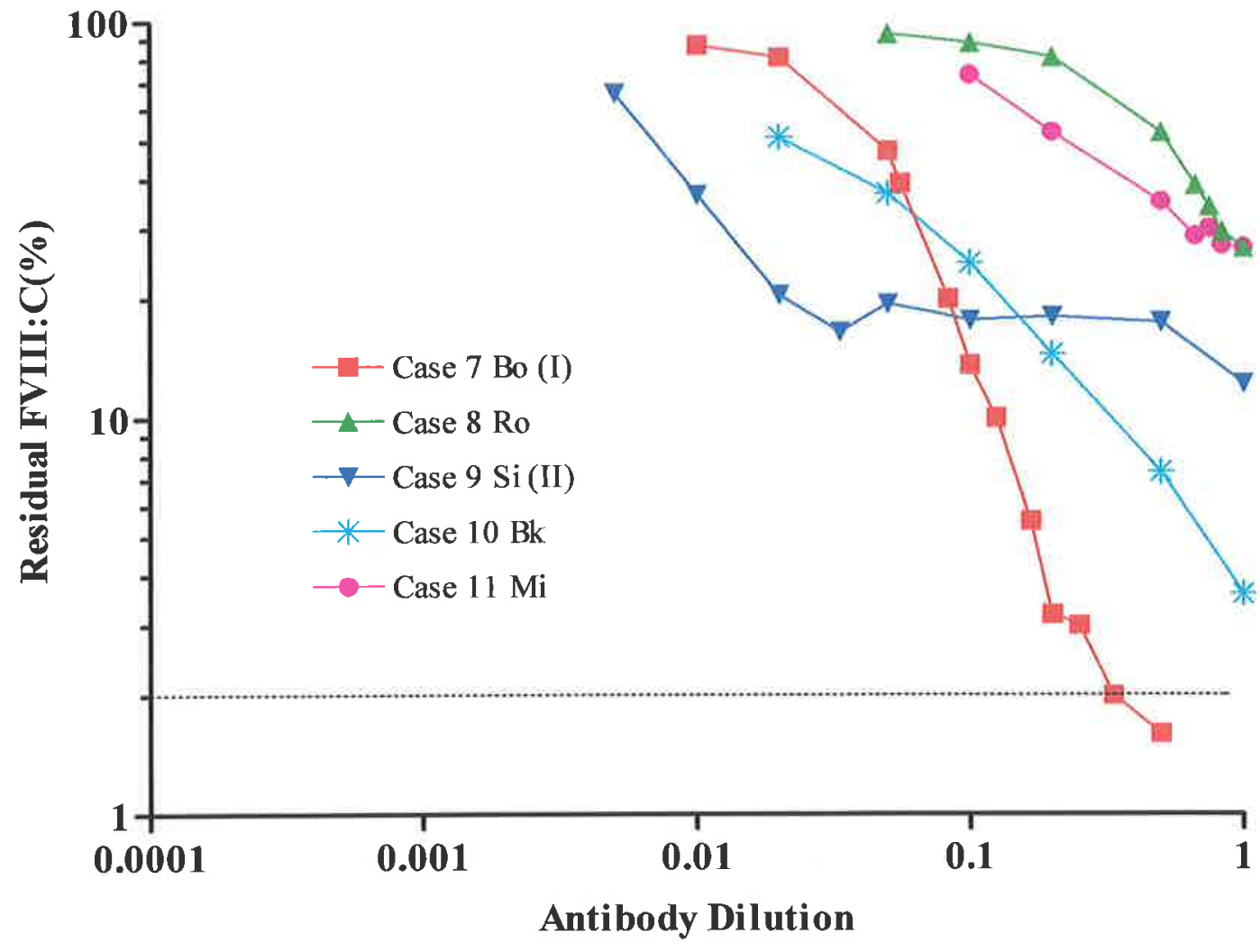

FIGURE 3.7 Effect of Antibody Concentration

(Cases 7-11)

A series of plasma dilutions for six cases were incubated for two hours with pooled normal plasma. Residual FVIII:C (\%) was plotted against antibody dilution, both on logarithmic scales. Case 7 was classified as having a type I inhibitor, while Case 9 was classified as having a type II inhibitor. Inhibitors from Case 8,10 and 11 were unable to be definitely classified because of the low titre.

The dashed line indicates $2 \%$ residual FVIII:C, which is the minimum detectable residual FVIII:C in the experiment (corresponds to FVIII:C level of $0.01 \mathrm{IU} / \mathrm{ml}$ ). 
TABLE 3.3 IgG Purification and Concentration of Six Plasma Samples

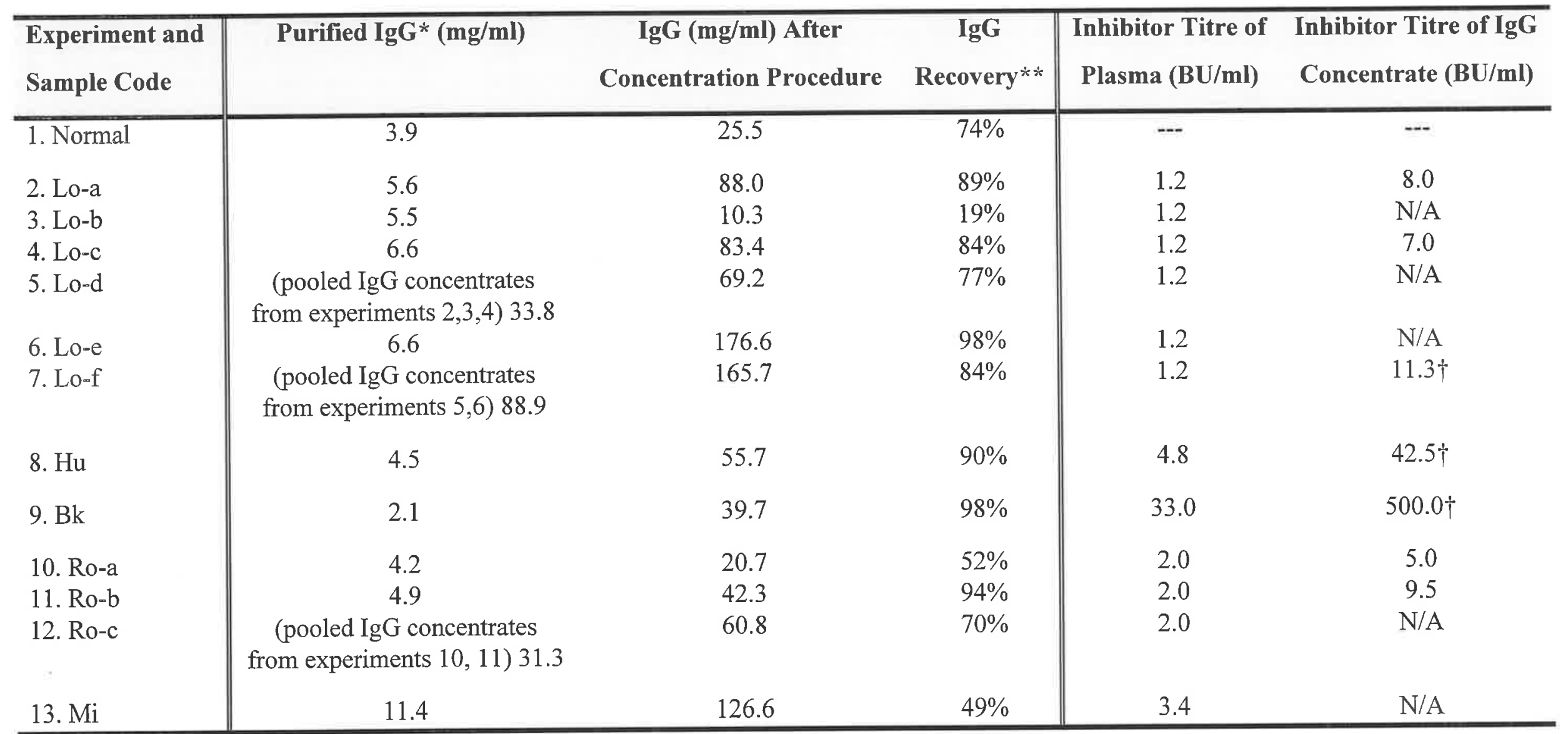

IgG purification and concentration was performed with six plasma samples, including four low-titre cases, one high-titre case (Bk) which could not be defined by concentration study, and one normal donor sample.

*: Pooled fractions 7, 8 and 9 eluted off sepharose-protein $\mathrm{G}$ column contained IgG.

$* *$ : Recovery calculated from the end of purification to the concentrated product.

$\dagger$ : Final inhibitor titre in IgG concentrate, with which the inhibitor type was defined.

N/A: Not available. 
not a plateau could be achieved on the graph of concentration versus residual FVIII:C. To try to solve the problem with these low-titre inhibitors, IgG was purified and concentrated from patient plasma, and thus a higher inhibitor level was achieved for use in the antibody dilution study. Table 3.3 shows the results of 13 individual experiments of IgG purification and concentration on six plasma samples. Four of them were low-titre cases (Case 3, 5, 8, and 11). One was a high-titre case (Case 10, BU=33) which could not be defined by a concentration study using a plasma sample, and one was normal blood donor sample. The yields during the concentration of purified IgG varied from experiment to experiment, with the range of approximately $50 \%$ to $90 \%$ recovery in these 13 individual experiments. The inhibitor titre in the concentrated IgG was found to be about 3-15 fold higher than the original titre in plasma.

Three inhibitors: Case 3, Case 5 and Case 10, were successfully defined all as type I using purified and concentrated IgG in this way. The classification of Case 8 and Case 11 however, remained undetermined at the end of the study due to insufficient plasma samples available for IgG purification. Figure 3.8 (Case 3, 5 and 10) gives examples of kinetic classification using purified and concentrated $\operatorname{IgG}$ in a study of the effect of antibody concentration. Case $3(\mathrm{Hu})$, who was a severe inherited case with baseline inhibitor titre of 4.8 $\mathrm{BU} / \mathrm{ml}$, was demonstrated to have a type I inhibitor by the antibody dilution curve, after increasing the inhibitor titre to $42.5 \mathrm{BU} / \mathrm{ml}$ by IgG purification and concentration. In another case of a patient with severe haemophilia (Case 5, Lo), the antibody concentration curve also displayed a type I pattern, when the inhibitor titre was elevated from $1.2 \mathrm{BU} / \mathrm{ml}$ to $11.3 \mathrm{BU} / \mathrm{ml}$. In this patient the final titre was only obtained after six purification and concentration steps, including the further concentration of pooled IgG concentrates from 


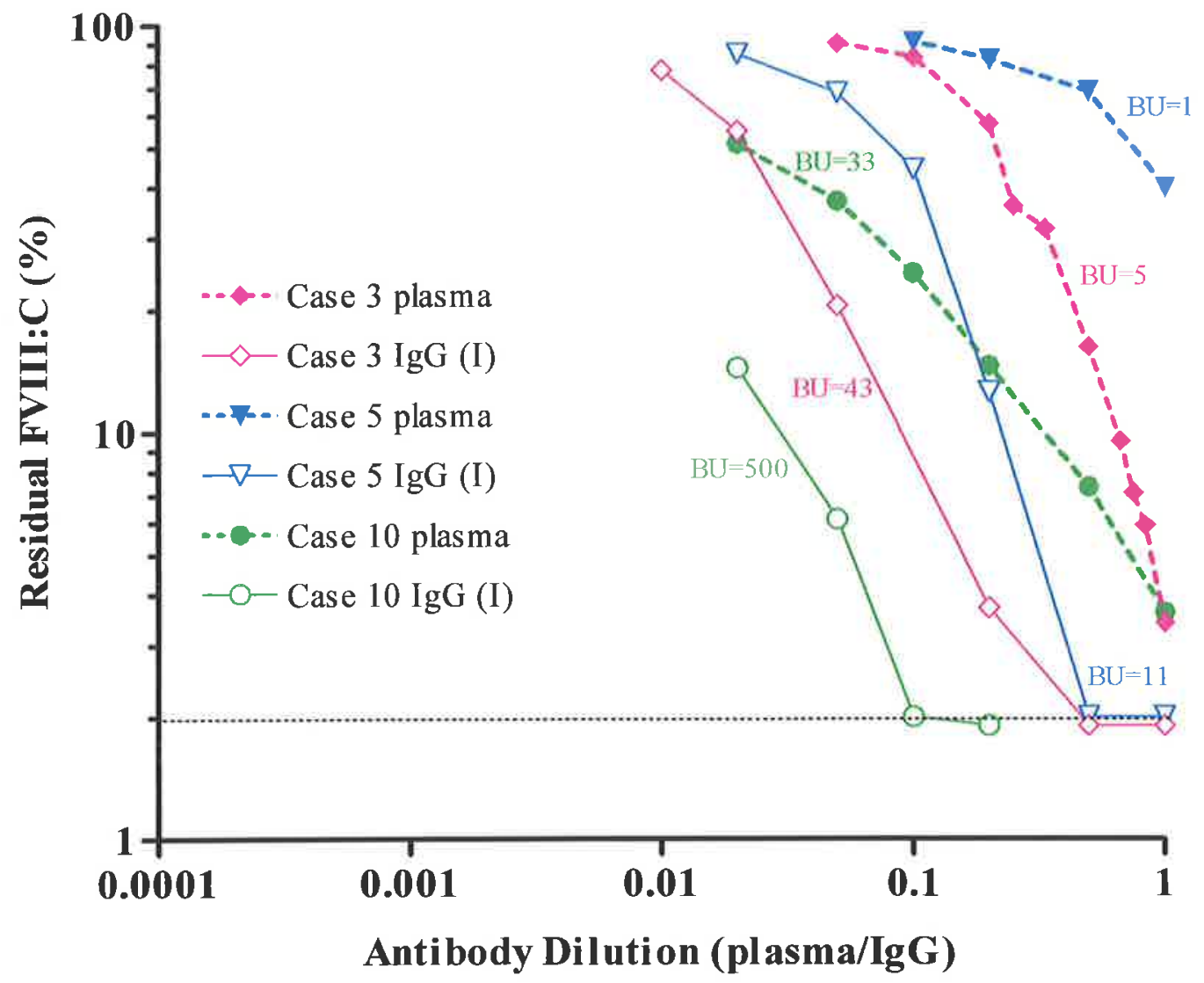

\section{FIGURE 3.8 Antibody Concentration Study Using Purified and Concentrated IgG}

IgG purification and concentration were successful in three cases (Case $3 \mathrm{Hu}$, Case $5 \mathrm{Lo}$, and Case $10 \mathrm{Bk}$ ) to obtain high enough inhibitor titre. The antibody concentration study using both plasma and IgG concentrate for these three cases was plotted. All show a type I kinetic pattern, with a complete inactivation of FVIII:C (residual FVIII:C $<2 \%$, i.e., below the dashed line). 
different experiments. Case $10(\mathrm{Bk})$ was a patient with acquired haemophilia A with a high baseline inhibitor titre of $33 \mathrm{BU} / \mathrm{ml}$. Although it appeared to be of the type I pattern from the antibody concentration curve, there was still greater than $2 \%(3.6 \%)$ residual FVIII:C when it was tested with full strength plasma. With IgG purification and concentration, an inhibitor titre of about $500 \mathrm{BU} / \mathrm{ml}$ was achieved. Complete inactivation was achieved confirming that it is a type I inhibitor.

\subsubsection{Mathematic analysis of the antibody concentration curves}

The data from the antibody concentration studies was analysed with GraphPad Prism nonlinear regression programs to find a model which would give a smooth curve through the data points and find the best-fit values in the equation. The sigmoidal dose-response (variable slope) model gave the best fit. The top value was set as the constant of 1.0 $(100 \%)$ which fits with the experiment condition as the dilution curve theoretically starts from the top point where the residual FVIII:C is $100 \%$. The equation is as follows:

$$
Y=\text { Bottom }+\frac{(\text { Top }- \text { Bottom })}{1+10^{(\operatorname{LogEC} 50-X) \cdot H i l l s l o p e}}
$$

When the Top value is set for the constant $1.0(100 \%)$, the equation changes to:

$$
Y=\text { Bottom }+\frac{(100 \%-\text { Bottom })}{1+10^{(\operatorname{LogEC} 50-X) \cdot \text { Hillslope }}}
$$

The $\mathrm{X}$ value is the logarithm of the antibody dilution, and the $\mathrm{Y}$ value is the residual FVIII:C in percentage. "Bottom" is Y value at the bottom plateau, and "LogEC50" is the antibody concentration required to compete for half the inactivation between "Bottom" and "Top (100\%)". "Hillslope" is unitless, and denotes the slope of the curve. "Hillslope" is 
TABLE 3.4 Results of Antibody Concentration Study from the Sigmoidal Dose-Response (Variable Slope) Model Analysis on 11 Patients

\begin{tabular}{|c|c|c|c|c|c|c|c|}
\hline$\longdiv { \text { Patient Code } }$ & Data Points & $\overline{\mathbf{R}^{2}}$ & Bottom & 95\% Confidence Interval of Bottom & $\overline{\mathrm{EC50}}$ & Hillslope & Kinetic Type \\
\hline 1. Du & 9 & 0.9955 & -0.02273 & -0.1154 to 0.05176 & 0.04677 & -1.630 & $\bar{I}$ \\
\hline 2. Go & 11 & 0.9918 & -0.03181 & -0.1154 to 0.05176 & 0.10659 & -1.923 & I \\
\hline 3. $\mathrm{Hu}$ & 10 & 0.9913 & -0.01731 & -0.1109 to 0.07628 & 0.21722 & -1.883 & \multirow[t]{2}{*}{ I } \\
\hline$H u \operatorname{IgG} *$ & 6 & 0.9977 & 0.01361 & -0.004804 to 0.03202 & 0.02153 & -1.647 & \\
\hline 4. Fi & 7 & 0.9703 & -0.4919 & -2.414 to 1.430 & 0.75007 & -1.489 & I \\
\hline 5. $\operatorname{Lo~IgG*}$ & 6 & 0.9884 & -0.02921 & -0.1903 to 0.1318 & 0.08072 & -1.536 & $I$ \\
\hline $6 . \mathrm{El}$ & 10 & 0.9940 & 0.06477 & 0.03717 to 0.09236 & 0.00137 & -1.984 & II \\
\hline 7. Bo & 12 & 0.9918 & -0.01597 & -0.06299 to 0.03105 & 0.04365 & -1.846 & I \\
\hline 8. Ro & 8 & 0.9943 & -0.2943 & -1.142 to 0.5533 & 0.75249 & -1.210 & I \\
\hline 9. Si (M) & 9 & 0.9860 & 0.1635 & 0.1391 to 0.1879 & 0.00592 & -2.262 & II \\
\hline 10. Bk (F) & 6 & 0.9977 & -0.04608 & -0.1311 to 0.03892 & 0.02489 & -0.684 & I \\
\hline 11. Mi (F) & 7 & 0.9965 & 0.2247 & 0.1652 to 0.2842 & 0.15031 & -1.492 & II \\
\hline
\end{tabular}

The results generated by the computer analysis using GraphPad Prism statistical program showing: $\mathrm{R}^{2}$ as an indication of goodness of fit; The best-fit values for Bottom (where curve plateaus out), EC50 (antibody dilution to give 50\% inactivation), and Hillslope (effectiveness of antibody); The $95 \%$ Confidence Interval of Bottom as the possible objective measurement to distinguish type I inhibitor (including zero) from type II (significantly positive from zero).

*: data obtained from purified and concentrated patient $\operatorname{Ig} G$, rather than plasma samples. 
negative in this study, which means the curve decreases as $\mathrm{X}$ increases. The Prism program calculated the best-fit values of the variables Bottom Value, EC50, and Hillslope. Table 3.4 shows the parameters obtained from the computer analysis.

The overall goodness of fit of the data was satisfactory. The best-fit values for the model given by the computer by analysing each set of data reflected that EC50 and Hillslope , which was the indication of the amount of antibody and the effectiveness of the antibody respectively, do not have a tendency to form any recognisable subgroups with different values corresponding to the different kinetic groups defined by later observation. However, the result of the Bottom Value of the model showed a definite difference, which divided the study population into two groups. For all the six type I inhibitors found with previous non-mathematic methods (three cases through plasma study, three through IgG study), the Bottom Value was always minus, with the $95 \%$ confidence interval of Bottom Value including the zero point. In contrast, for the two defined type II inhibitors, the Bottom Value was around $10 \%$ (one $6 \%$ and one $16 \%$ ), and the $95 \%$ confidence interval did not include zero.

This discovery was important, suggesting that the two types of factor VIII inhibitors could possibly be objectively classified by deciding the parameters of the Bottom Value of the sigmoidal dose-response (variable slope) model. To explore this observation, the antibody dilution study data of the remaining three patients with low inhibitor titre and undefined kinetic feature were analysed using the same model. The three patients were Case $4(\mathrm{Fi}$, severe inherited haemophilia with intron 22 inversion, $\mathrm{BU}=3$ ), Case 8 (Ro, mild inherited haemophilia, BU=2) and Case $11(\mathrm{Mi}$, acquired haemophilia, BU=3). The result was 
TABLE 3.5 Summary of 11 Haemophilia A Patients in the In Vitro Kinetic Studies

\begin{tabular}{|c|c|c|c|c|c|c|}
\hline $\begin{array}{l}\text { Patient } \\
\text { Code } \\
\end{array}$ & Status & $\begin{array}{c}\text { Gene } \\
\text { Mutation } \\
\end{array}$ & $\begin{array}{c}\text { Plasma } \\
(\mathrm{BU} / \mathrm{ml}) \\
\end{array}$ & $\begin{array}{c}\text { IgG } \\
(\mathrm{BU} / \mathrm{ml}) \\
\end{array}$ & $\begin{array}{c}\text { Kinetic Type } \\
\text { Observed } \\
\end{array}$ & $\begin{array}{c}\text { Kinetic Type } \\
\text { by Maths }\end{array}$ \\
\hline $1 . \mathrm{Du}$ & Severe Inherited & large del. (exon 21) & 30 & & $\overline{\mathrm{I}}$ & $\bar{I}$ \\
\hline 2. Go & Severe Inherited & intron 22 inv. & 13 & & I & $\mathrm{I}$ \\
\hline $3 . \mathrm{Hu}$ & Severe Inherited & intron $22 \mathrm{inv.}$ & 5 & 43 & $I^{*}$ & I \\
\hline 4. Fi & Severe Inherited & intron 22 inv. & 3 & $N / A$ & $? ?$ & $I$ \\
\hline 5. Lo & Severe Inherited & intron 22 inv. & 1 & 11 & $I^{*}$ & I \\
\hline 6. $\mathrm{El}$ & Mild Inherited & Arg $2150 \mathrm{His}$ & 700 & & II & II \\
\hline 7. Bo & Mild Inherited & Tyr 2105 Cys & 23 & & I & I \\
\hline 8. Ro & Mild Inherited & Arg 2150 His & 2 & 10 & $? ?$ & $I$ \\
\hline 9. Si (M) & Acquired & --- & 140 & & II & II \\
\hline 10. Bk (F) & Acquired & -- & 33 & 500 & $I^{*}$ & I \\
\hline 11. $M i(F)$ & Acquired & - & 3 & $N / A$ & $? ?$ & II \\
\hline
\end{tabular}

Kinetic type of factor VIII inhibitors was defined basically through the study of effect of antibody concentration.

Inhibitors in patients $1,2,6,7$, and 9 were defined type I or type II by both observation on plasma results and mathematic analysis.

Inhibitors in patients 3,5 , and 10 were defined type I or type II by both observation on IgG results (*) and mathematic analysis. Inhibitors in patients 4, 8, and 11 were defined type I or type II by mathematic analysis alone.

del.: deletion; inv.: inversion; N/A: not available; *: result obtained with IgG instead of plasma. 
encouraging. For Case 11, the only acquired patient among the three, a positive Bottom Value of $22 \%$ and the confidence interval of $16-28 \%$ were seen, which was a typical mathematical feature for the type II. This result was clearly different from the results of Case 4 and Case 8 , in which the typical type I pattern was shown as a minus Bottom Value and a confidence interval including zero. The results of the classification by this mathematical analysis strengthen the confidence in the judgement of the type from visual observation of the curves. A summary of the kinetic classification of factor VIII inhibitors in 11 patients based on in vitro antibody concentration study was shown in Table 3.5.

\subsection{Effect of Extra Source of Factor VIII}

The aim of this section was to determine whether further inhibitor activity can be detected in incubations that have reached their maximum degree of inhibition of FVIII:C, i.e., after equilibrium is reached. This is pertinent to the study of type II inhibitors which are characterised by incomplete inactivation of FVIII:C after their incubation with normal plasma, even when very high concentrations of inhibitor are present. The study of Biggs et al. (1972 b) briefly described data for one patient with acquired haemophilia, where it was shown that addition of more factor VIII during the slow phase of factor VIII destruction was followed by rapid destruction of the newly added factor VIII.

In these experiments AHF was used as the source of factor VIII, as described in Chapter 2, section 3.1.5. The initial starting concentration of factor VIII in the incubation mixture with patient samples was $1.0 \mathrm{IU} / \mathrm{ml}$. After four hours incubation, and based on the assay result at that time, a small volume (approximately 10-20 $\mu 1$ ) of AHF was added in an amount calculated to adjust the factor VIII:C in the incubation mixture back to $1.0 \mathrm{IU} / \mathrm{ml}$. 
The results in Figure 3.9 show two different patterns, one typical of type I (Case 1) inhibitor, the other typical of type II (Case 9) inhibitor.

In Case $1(\mathrm{BU}=30)$, after the addition of an extra amount of $\mathrm{AHF}$ at the end of four hour incubation, there was no obvious inactivation of additional FVIII:C over the subsequent four hours. Case $9(\mathrm{BU}=140)$, which was a type II inhibitor case, however, gave a significant further inactivation of the extra FVIII:C (in plasma dilution 1/50). Both chosen dilutions of the two plasma samples brought the residual factor VIII:C in each mixture to a similar level after four hours incubation. This observation could be summarised as type I inhibitor can not neutralise additional FVIII:C after equilibrium is reached, while type II inhibitor can (Biggs et al., 1972; Bovill et al., 1985). In this study, the results were consistent with the classification result of these two cases by antibody concentration studies as described above.

However, misleading results were seen when different dilutions of plasma samples were used. As shown in Figure 3.10 (a), the same type I inhibitor (Case 1) gave a false "further inactivation" of extra FVIII:C when it was too much in excess of antigen in the incubation mixture. In this experiment, three different dilutions $(1 / 4,1 / 6$, and $1 / 10)$ of plasma of Case 1 were tested. With the dilutions of $1 / 4$ and $1 / 6$, a further inactivation of additional FVIII:C was observed, due to the excess amount of antibody in the reaction mixture. The result with dilution 1/10 remained the same as the previous experiment, showing no further inactivation, as characterised for type I kinetics. 


\section{FIGURE 3.9 Study of Effect of Extra Source of Factor VIII}

Patient plasma samples (Case 1, 1/10 dilution and Case 9, 1/50 dilution) were incubated with pooled normal plasma for an extended time course study. For the control, commercial factor VIII deficient plasma (Organon, Teknita) was used instead of inhibitor plasma. After four hours incubation, an extra amount of AHF was added, and the reaction mixture was incubated for another four hours. Remaining FVIII:C (IU/ml) was measured at different time points, and plotted against incubation time, both on linear scales.

*: reaction mixture with extra source of factor VIII. 


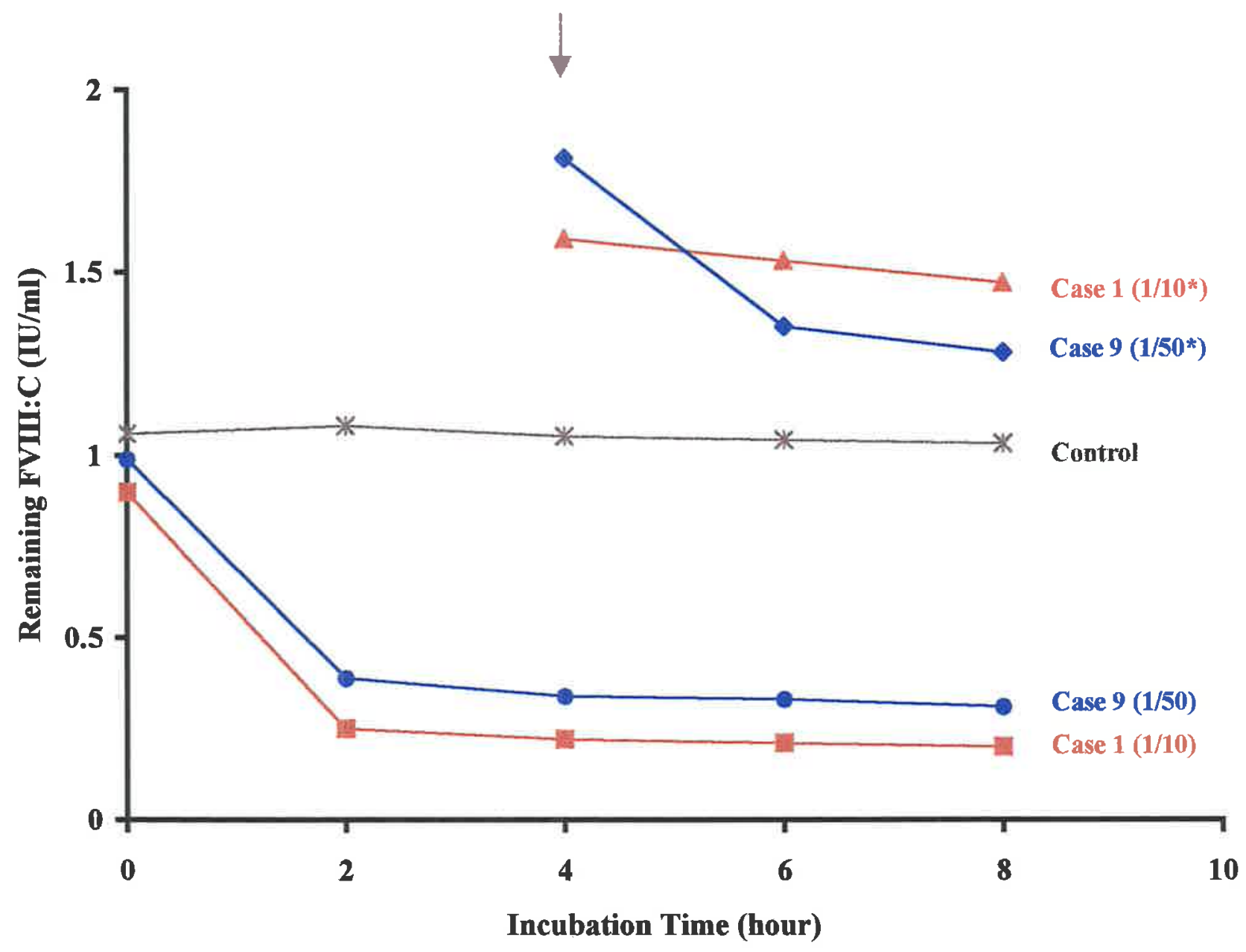


a

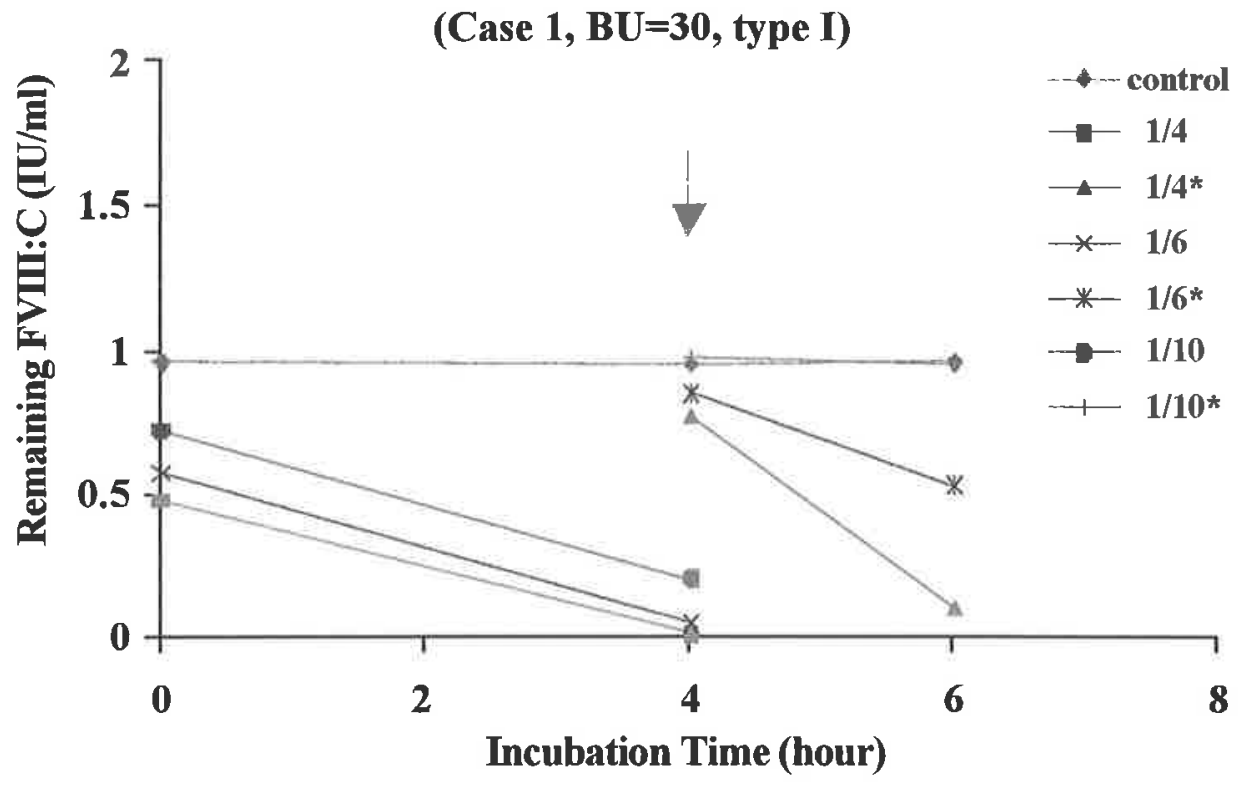

b

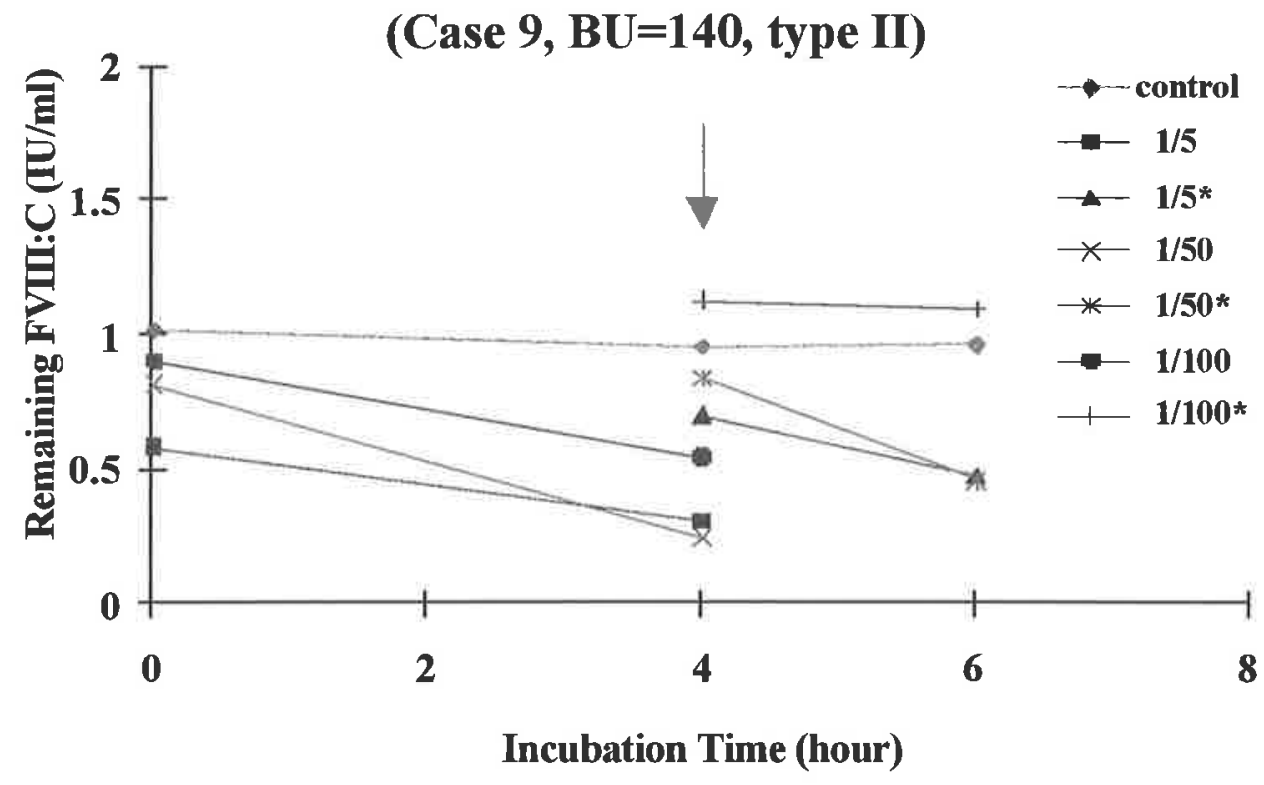

FIGURE 3.10 Further Study of Effect of Extra Factor VIII

Case 1 (a) and Case 9 (b) were studied in more plasma dilutions. The methods were the same as described before for Figure 3.9. After four hours incubation, an extra amount of AHF was added, aiming to bring the FVIII:C level in the reaction mixture back to $1.0 \mathrm{IU} / \mathrm{ml}$. Remaining FVIII:C (IU/ml) was measured during the time course. ${ }^{*}$ : reaction mixture with the extra source of factor VIII. 
Similarly, for a type II inhibitor (Case 9), when the antibody concentration was too low, no inactivation of the added factor VIII was observed. As shown in Figure 3.10 (b), the plasma of Case 9 were tested with three dilutions: $1 / 5,1 / 50$, and $1 / 100$. With the dilutions $1 / 5$ and $1 / 50$, it showed a further neutralisation of extra factor VIII, consistent with the previous result. But with the dilution of $1 / 100$, there was no obvious inactivation of additional factor VIII, as the antibody amount was not sufficient to show such a change in a gross level. Therefore the results of this method are dependent on the selection of a critical level of inhibitor for the incubation mixture. This renders the method impractical for routine application to distinguish type I and type II inhibitors.

\subsection{Effect of Initial Factor VIII Concentration}

The aim of this section was to determine the effect on kinetics of reducing the initial factor VIII level in the incubation mixture. A reduction in the initial factor VIII concentration will alter the ratio of antibody:antigen, and should provide antibody in excess of antigen. We considered that this may increase the sensitivity to the effect of inhibitors, and facilitate the demonstration of different kinetics for type I and type II. However, Biggs et al. (1972 b) was not successful in demonstrating that kinetic behaviour could depend on initial factor VIII level.

The study of the initial factor VIII concentration in the reaction mixture, was carried out with two severe inherited (Cases 1 and 5) and two acquired (Cases 9 and 11) patient samples. Each inhibitor plasma was tested at four different dilutions, and each of these dilutions was tested against four dilutions of pooled normal plasma. The four dilutions of normal plasma therefore provided four different initial factor VIII concentrations. Residual 
FVIII:C in the mixture was measured after two hours. Figure 3.11 shows the result of the four cases studied, with the initial factor VIII (IU/ml) plotted on $\mathrm{X}$ axis, and the residual FVIII:C (\%) on Y axis. The four different concentrations of initial factor VIII tested were full strength, 2:3, 1:2, and 1:4 dilutions of routine pooled normal plasma. As observed from Figure 3.11, regardless of the initial factor VIII concentration, for a given dilution of a patient plasma, there was always a fixed percentage of FVIII:C destroyed within a two hour incubation. For each of the four patients, we studied four different antibody dilutions, which covered a reasonable range of inhibitor strength. The degree of the inhibition was proportioned to the strength of the antibodies. There was as expected, a decrease in the residual FVIII:C correspondent to an increase in the antibody level.

Figure 3.12 (Case 1) shows the results when the residual FVIII:C (\%) is plotted against the antibody dilution, instead of the initial factor VIII concentration on X axis. It is clearly seen from the graph that the four dilution curves with different initial factor VIII level are overlapping each other. This again reflects that the adjustment of the initial factor VIII level does not have a significant effect on the residual factor VIII:C (\%). The results from the other three cases studied show the same pattern plotted in this way. Therefore, decreasing the initial factor VIII level did not increase the sensitivity for detection of the inhibitors.

\section{Chapter Discussion}

Biggs et al. (1972 a and b) and Gawryl and Hoyer (1982) mainly used study of time course and antibody concentration to examine the two different types of kinetics of factor VIII inhibitors. The validity of those methods has not been studied in detail. In addition, no 


\section{FIGURE 3.11 Study of Effect of Initial Factor VIII Concentration (four cases)}

For each case, four different patient plasma dilutions were incubated for two hours with an equal volume of four different concentrations of initial factor VIII, i.e., full strength, 2:3, 1:2 and 1:4 dilutions of routine pooled normal plasma. The residual FVIII:C (\%) is plotted on log scale against the initial FVIII:C (IU/ml) on linear scale. FS: full strength (of patient plasma). 

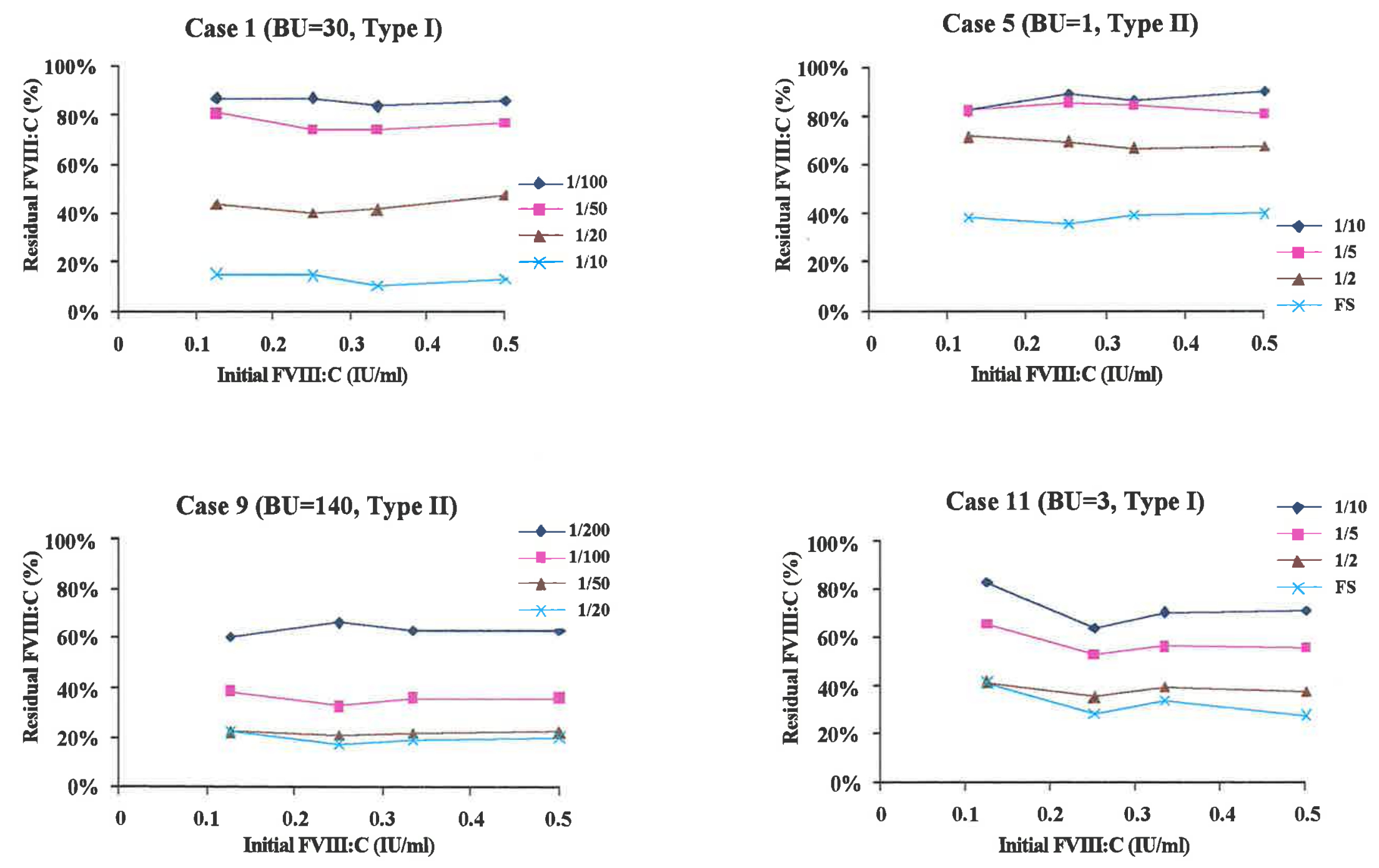


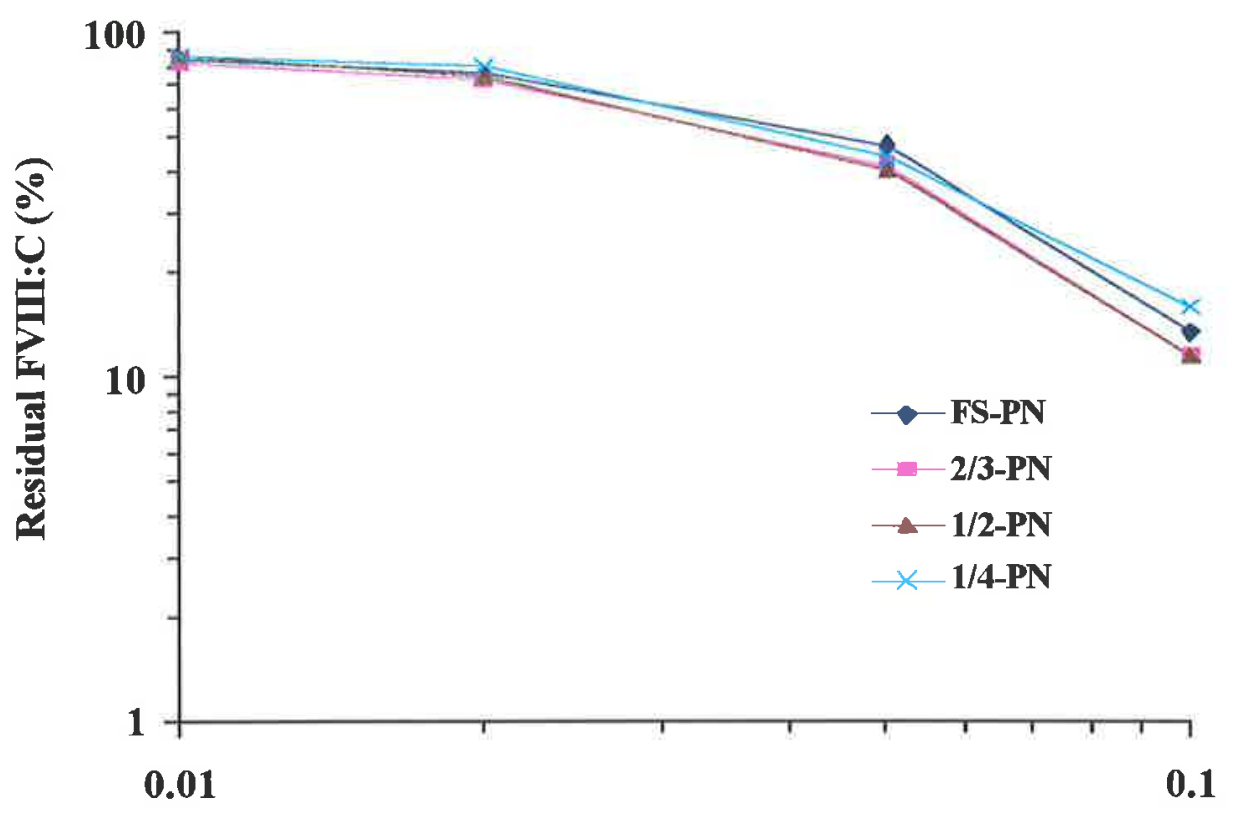

Antibody Dilution

\section{FIGURE 3.12 Study of Effect of Initial Factor VIII Concentration (Case 1)}

Plasma sample from Case 1 was tested with four antibody dilutions: $1 / 100$, $1 / 50,1 / 20$ and 1/10. Each antibody dilution was incubated with four different dilutions of pooled normal plasma (full strength, 2/3, 1/2 and 1/4), in order to obtain different initial factor VIII level. The residual FVIII:C (\%) after two hours was plotted against antibody dilutions, both on log scales. The four curves observed were overlapping with each other, even though the initial FVIII:C level in each was different.

FS: full strength. PN: pooled normal (plasma). 
one has published a study of a group of patients by both these methods. In this project, in order to examine the in vitro kinetics of inactivation of factor VIII by factor VIII inhibitors, the effects of incubation time, antibody concentration, the addition of extra factor VIII and varying the initial factor VIII level were studied in the patient group of 11 haemophilia A cases. These methods are different ways to explore the behaviours of the inhibitors in their interaction with FVIII:C. In the studies of the effect of incubation time, there was no significant differences in the patterns of inactivation among the nine patient samples studied, both by visual observation of the time course curve and the mathematical analysis of the experimental data. This suggested that, with the experimental methods used here, the study of effect of incubation time will not be helpful to distinguish the kinetic features of the two types of factor VIII inhibitors. Only one approach, the effect of antibody concentration, was useful to classify inhibitors as type I and type II. Table 3.2 shows the comparison of the analysis of time course results generated by mathematical analysis, with the final classification results determined by the antibody concentration study.

The results showed that the most promising way to differentiate two types of factor VIII inhibitors based on their in vitro kinetic behaviour is the antibody dilution study, in which two distinctive patterns of FVIII:C inactivation can be seen when sufficiently high amounts of antibody are present. This study was consistent with previous studies (Gawryl and Hoyer, 1982) as it demonstrated that type I inhibitor completely neutralises all available FVIII:C (>98\%) at high antibody concentrations. Also the type II inhibitors in this study have more complex kinetics and cannot neutralise all available FVIII:C even when tested undiluted, so a curvilinear pattern with a plateau at high antibody concentration is typical. 
Three inherited haemophilia A patients (two severe, one mild) were classified as having type I inhibitors, and two (one mild inherited case and one acquired case) as having type II inhibitors, by studying the effect of antibody concentration using patient plasma. Generally, samples with $>10 \mathrm{BU} / \mathrm{ml}$ inhibitor could be classified on the basis of an antibody dilution study using plasma. Nevertheless, one patient was an exception and although the plasma contained more than $10 \mathrm{BU} / \mathrm{ml}$ inhibitor titre, it still could not be defined by the dilution study with plasma (Case 10 in this study). Three more type I inhibitors (two severe cases and one acquired case) were defined later using purified, concentrated IgG to study the effect of antibody concentration. With the GraphPad Prism statistical program, the three remaining undetermined samples were classified using extrapolation of the concentration curve. In our small patient population, both types I and II were found in the mild/acquired group. This study is therefore consistent with the general concept that factor VIII inhibitors that develop in severe haemophilia A patients are usually type I, and those whose inhibitors form in mild haemophilia cases may be either type I or type II (Gilles et al.,' 1997; Hoyer and Scandella, 1994; Pruthi and Nichols, 1999). In the acquired haemophilia inhibitor patient group $(n=3)$, one was determined to be type I and the others were type II. Although it is possible that type I inhibitors may be more prevalent among acquired haemophilia than previously realised, this should be clarified in a larger study.

Use of the mathematical formula for a sigmoidal dose response curve to calculate the Bottom Value of the curve has provided useful data for classifying the kinetic properties of the two types of factor VIII inhibitors. All cases of type I inhibitors gave a negative Bottom Value. However, the 95\% confidence interval for that value included zero, 
indicating that it did not differ significantly from zero. In contrast the concentration curves for type II cases were characterised by having both a Bottom Value and its $95 \%$ confidence interval significantly above zero. This criteria led to a classification consistent with the result obtained by using observation of the antibody concentration curves of those inhibitors which were high titre. For inhibitors which were low-titre, again, this mathematical formula gave the same classification as the result gained from an antibody dilution study using purified and concentrated inhibitor IgG. In addition, for the three cases in this study (Cases 4, 8 and 11) whose inhibitor type had remained unidentified because it was not possible to obtain the antibody in high titre, this formula was also able to classify their kinetic type as well. This was achieved by examining the best fit Bottom Value and the $95 \%$ confidence interval of the value generated by the computer program.

The mathematically calculated result for a type I inhibitor, showing that the bottom of the curve tends towards zero, is in keeping with the complete inactivation of FVIII:C observed visually on the antibody concentration curve. A positive Bottom Value shown by a type II inhibitor, is in keeping with its inability to completely neutralise factor VIII even when at high antibody concentrations. This suggests that the use of a mathematic formula to classify factor VIII inhibitors based on antibody concentration studies, is a useful and objective method of analysis. This mathematical method is therefore, a reasonably convincing and objective way to distinguish the two types of factor VIII inhibitors. It is important however, to mention that the mathematical formula is an extrapolation of the curve based on the limited data which was obtained from plasma. Therefore, interpretation by this mathematical analysis should be cautious if the number of data points is not sufficient. The application of this method in a larger number of patients is still required in 
order to confirm the reliability of this method. This may be difficult to achieve due to the relatively low number of inhibitor patients in the population. Nevertheless, from our point of view, the goodness of the fit of the data and the reasonable classification result for each of the 11 patients in this study, have given sound supportive information to the evaluation of this mathematical analysis.

We concluded that, in order to classify the kinetic type of factor VIII inhibitors, the use of an antibody concentration study is an efficient and readily standardised method for those high-titre inhibitors. To solve the problem of those low-titre inhibitors, which are becoming more common due to improved management of the patients (Lloyd et al., 1997), the approach of using purified and concentrated IgG to examine the effect of antibody concentration is useful. The use of mathematical formula analysis, can also be considered as a part of the assessment of the result. Table 3.5 summarises the final classification for our patients, achieved using the antibody concentration studies.

Type II inhibitors do not completely inactivate all available FVIII:C, even when tested undiluted. This kinetic property is consistent with the in vivo finding that in some patients, there is always detectable FVIII:C level, even though there is also a significant inhibitor titre (Rizza and Biggs, 1973). The reasons behind such differences in neutralisation of FVIII:C by the two types of inhibitors is not elucidated.

There are two possible hypotheses to account for these differences. One is that, type I inhibitors have high binding affinity for the factor VIII molecule, while type II inhibitors have relatively low affinity binding (Allain and Frommel, 1974; Gilles et al., 1997; Hoyer 
and Gawryl, 1984; Kasper, 1984). This hypothesis explains well the effect on an extra amount of factor VIII added during the time course experiments (Figure 3.9). Because type II inhibitors bind loosely to factor VIII, it is possible for them to dissociate from the original factor VIII molecules, and bind to the factor VIII molecules which also become inactivated. This hypothesis would imply that there is a permanent inactivation of FVIII:C by the type II antibodies. Hence the original factor VIII remains inactivated after it has dissociated from the antibody. An alternative explanation for the type II kinetics is that, those bound antibodies can remain bound but still inactivate further factor VIII. This seems inherently unlikely. However, there is no direct experimental evidences to verify either possibility.

Of interest is the plateau which is found in patients with type II inhibitors in the antibody concentration studies. A possible explanation for this is that type II antibodies might only partially inhibit function. This could for instance occur if they blocked only part of an important functional site. For instance it is likely that phospholipid binds to multiple sites in the C2 domain. An inhibitor directed at only some of these sites would likely not produce a complete inhibition. Nevertheless, the finding by Gawryl and Hoyer (1982) that removal on protein-A-sepharose of antigen-antibody complex did not reduce factor VIII activity in the mixture, argues against this hypothesis. In future studies, it would be interesting to explore this hypothesis further, perhaps by repeating the experiments by Gawryl and Hoyer.

It is possible that in some patients there may be a mixture of both type I and type II inhibitors. In support of this, some researchers have demonstrated that human polyclonal 
antibodies behaving as type I inhibitors could be converted to type II after neutralisation of the type I inhibitors in the mixture by addition of anti-idiotypic antibodies (Gilles et al., 1997).

In our experiments, type I and type II inhibitors could not be distinguished from the results of the time course study. This is quite different from Biggs' conclusion. Nevertheless, in re-examining our data and reviewing Biggs' paper, it seems likely that she would have only observed a rapid initial linear decline (Figure 3.1 a) when the antibody was in excess of antigen. The difficulty with this concept from a practical point of view was that it is difficult to define when antigen or antibody is in excess. In our time course experiments, the percentage residual FVIII:C measured after two or four hours incubation were similar to those seen by Biggs, suggesting a similar ratio of antigen to antibody. For each individual patient sample, at least two antibody concentrations were selected for the experiment, in order to ensure a reasonable and consistent range of the ratio of antibody:antigen in the reaction mixture. Therefore, it seems unlikely that our failure to detect a linear pattern was due to our choice in inhibitor concentration.

The results of effect of incubation time in this study not only disagree with Biggs' criteria, but are also difficult to interpret in the light of the results of the two other main studies. Among the few papers with detailed experiments carried out to study the kinetics of factor VIII inhibitors in a small patient group, Allain and Frommel (1974), and Green et al. (1999) claimed to show a typical pattern for type I inhibitor in a time course studies. However in Green's study the difference claimed between type I and type II were based on only marginal difference in statistical fit between a straight line and an exponential curve. 
In Allain's study the differences between the two types depends on a value judgement relating to the steepness of the slope of the supposed plateau phase. Neither of the concepts in these two studies can be applied to our findings.

In our study the time course experiments did not differentiate the inhibitors into two types. This is in contrast to the very definite classification achieved in our studies of the effect of antibody concentration. The reason for the failure of the time course experiments to demonstrate any difference between the subjects may partially be that our experiments were carried out under different conditions compared to the methods used by Biggs et al. (1972, a and b), Allain and Frommel (1974), and Green et al. (1999). For instance, pooled normal plasma was used instead of human intermediate-purity factor VIII as a factor VIII source in our test, which may cause different antigenic exposure and antigen-antibody binding characters. Another important hypothesis could be that, the inhibitor patients in this study were different from those in the other studies. AHF (High Purity, CSL) is used as the only plasma-derived factor VIII concentrate in Australia, and this concentrate may differ antigenically from factor VIII used in other patient groups around the world.

The results of our study showing no relationship between the classification by time course and the classification by concentration effect shows for the first time that there are weaknesses in previous concepts of classification. A robust classification scheme would produce a consistent classification in all groups of patients. So far there is no explanation with supportive experimental evidence to clarify the causative reasons for the different results between our study and the previous publications. Re-examination of the previous methods used to classify inhibitors did show difficulties in comprehension, execution and 
interpretation of the results. However, our study is the first, from the practical point of view, to carry out a systematic study of in vitro kinetics of factor VIII inhibitors to classify inhibitor types in a small but diverse group of patients with haemophilia A. A successful approach for a routine and research coagulation laboratory to classify factor VIII inhibitors based on in vitro kinetics has been established, based on the antibody concentration study.

Some of the other possible kinetic features of the factor VIII inhibitors were also explored in this study, with some alternative experiments carried out in attempt to classify low-titre inhibitors. One method is to examine the effect of extra source of factor VIII. Biggs et al. (1972 b) once described a type II inhibitor showing a rapid destruction of the newly added factor VIII during the slow phase of the reaction. However, in the same publication work, they claimed contradictorily that, in both reaction mixtures involving type I and type II inhibitors, most of the antibody activity had been neutralised by the first addition of factor VIII. Thus there was no significant destruction of added FVIII:C by either type of these inhibitors, as demonstrated by one of their experiments (Biggs et al., 1972 b).

Also, in a case study of a type II inhibitor in a mild haemophilia patient in 1985 , the effect of adding an extra source of factor VIII after reaching the reaction equilibrium was studied (Bovill et al., 1985). The addition of factor VIII concentrate to the reaction mixture after four hours incubation led to a significant further neutralisation of FVIII:C over the subsequent four hours. This was explained to be due to the incomplete inactivation of FVIII:C by type II inhibitor in the first four hours incubation, which is consistent with the clinical picture of incomplete inactivation of FVIII:C following large dose of factor VIII concentrate administration in this patient (Bovill et al., 1985). 
In our extended time course study to examine the effect of an extra source of factor VIII, a type II inhibitor did neutralise additional FVIII:C after equilibrium was reached, while a type I inhibitor can not. This is consistent with the concepts that type II antibodies are only loosely bound with antigen. But the experimental conditions required to demonstrate such kinetic difference are so critical that the interpretation of results is not straight forward. In most situations, if the amount of antibody and factor VIII does not satisfy the certain ratio, i.e., too much antibody in a type I mixture, or too much antigen in a type II mixture, the experiment will give misleading results.

Another way to examine the kinetic features of the two types of factor VIII inhibitors is the study of initial factor VIII concentration. As a strategy alternative to purification and concentration of the antibody, we explored the use of a lower initial factor VIII concentration to increase sensitivity. In our studies, a fixed percentage of FVIII:C destroyed within two hour incubation was always seen with a given patient plasma dilution, no matter what the initial factor VIII concentration was, and what the type of inhibitor was. This was disappointing because we had expected that at lower initial factor VIII level, there would be a steeper decline with increasing antibody concentration.

\section{Chapter Summary}

In this in vitro kinetic study of factor VIII inhibitors, a variety of methods were used to characterise the kinetic features of the factor VIII inhibitors from 11 haemophilia A patients, including five severe, three mild and three acquired cases. By re-examining the 
previous described methods and exploring possible new alternatives, an approach to classify factor VIII inhibitors based on their in vitro kinetics has been established.

- Type I and type II factor VIII inhibitors can be distinguished by antibody concentration studies. This requires a relatively high level of inhibitor $(>10 \mathrm{BU} / \mathrm{ml})$. Purified and concentrated IgG concentrate is helpful for those weaker antibodies. On an antibody concentration curve, type I inhibitor is characterised by a steep slope and complete $(>$ 98\%) inactivation of FVIII:C at higher antibody concentrations, while type II inhibitor gives a plateau and incomplete inactivation even at higher antibody concentrations.

- The use of a mathematical program (GraphPad Prism) to analyse the dilution curve data with the sigmoidal dose-response (variable slope) model is useful to objectively distinguish the two types of inhibitors. The $95 \%$ confidence interval of the Bottom Value of the equation can be used as a criteria for discrimination: there is a zero for type I and a positive result for type II.

- Eight type I inhibitors were found with five severe cases, two mild cases, and one acquired case, using a combination of both observation and mathematical methods. At the same time, three type II inhibitors were found with two mild cases and one acquired case.

- Using the Bethesda method as the basis of experiments, other alternative ways, including the time course study, study of effect of extra factor VIII, study of initial factor VIII level etc., were not successful for classification of factor VIII inhibitors. 


\section{CHAPTER FOUR}

\section{ELISA METHOD TO DETECT FACTOR VIII ANTIBODIES}

\section{Introduction}

The term "inhibitor" is used for antibodies detected with functional assays such as the Bethesda and New Oxford assays (Mondorf et al., 1994). These methods measure the ability of those antibodies to bind functional epitopes involved in coagulant activity. It is not known whether, or to what extent, post-transfusion antibodies may also arise against epitopes which are not essential for function. However, there is evidence of the existence of antibodies capable of binding to factor VIII, but devoid of neutralising activity (Gautier et al., 1996; Mondorf et al., 1994). In these studies, immunoassays such as ELISA were used to detect factor VIII antibodies.

Theoretically, immunoassays that are based on the interaction between immunoglobulins and the antigenic sites on factor VIII molecules are able to detect both inhibitory and noninhibitory antibodies. ELISA is one type of immunoassays that have been developed to detect the binding of factor VIII antibody to factor VIII. The results of ELISA showed a good correlation with the Bethesda assay in a report of a study on 18 patients with haemophilia and five normal subjects (Regnault and Stolz, 1994). In contrast, another study showed that in a group of patients with inherited haemophilia A, 36\% (8 out of 22) had a positive ELISA result, but all were without a positive Bethesda titre (Dazzi et al., 1996). Furthermore, using the method of ELISA in severe haemophilia A patients with intron 22 inversion, the same group reported a high prevalence ( $81 \%, 13$ out of 16$)$ of antifactor VIII antibodies (Vianello et al., 1997). When the Bethesda assay was used, only $25 \%$ of these patients were found to have inhibitors. 
The clinical impact of such non-inhibitory antibodies has yet not been fully elucidated. However, it is possible that they could bind to factor VIII and cause it to have a reduced half-life in the circulation. The assessment of anti-factor VIII antibodies solely by a functional assay such as the Bethesda assay may not be adequate for the complete evaluation of the overall antibody response. It was therefore important to determine in our patients whether antibodies to non functional sites on the factor VIII molecule could be detected. We studied a group of patients with haemophilia by both an ELISA assay and the Bethesda assay. The aim was to determine whether there was a relationship between the results of these two assays. A positive titre by ELISA in a patient who was negative by the Bethesda method would be evidence for an antibody against a non-functional epitope.

The specific aims were:

1) To set up an ELISA method suitable for a routine or research coagulation laboratory to detect factor VIII antibodies, using commercial recombinant factor VIII product.

2) To investigate a number of haemophilia A patients, including a group of patients with intron 22 gene inversion, using the ELISA method. Compare the results with those obtained from the Bethesda assay, in order to determine if there is a correlation between the two methods in terms of detecting factor VIII antibodies.

3) In a group of severe haemophilia patients, to compare the ELISA inhibitor results for patients with the intron 22 gene inversion to the results for patients without the inversion. 


\section{Methods}

\subsection{Establishment of ELISA to Detect Factor VIII Antibody}

The ELISA method is described in Chapter 2, Section 3.2. To set up the method, a few experimental conditions were tested and then optimised, including the concentration of factor VIII to coat the plates and the buffer system. Recombinant factor VIII (Recombinate $^{\mathrm{TM}}, \mathrm{CSL}$ ) (stock concentration $50 \mathrm{IU} / \mathrm{ml}$ ) was diluted in different final concentrations $(1 \mathrm{IU} / \mathrm{ml}, 5 \mathrm{IU} / \mathrm{ml}$ and $10 \mathrm{IU} / \mathrm{ml})$ in both carbonate buffer and PBS buffer, and was used to coat the microtitre plates. Monoclonal anti-factor VIII antibody ESH 8 (American Diagnostica Inc.), which is directed to the C2 domain of the light chain (aa 2248-2285) (stock inhibitor titre $>5,000 \mathrm{BU} / \mathrm{ml}$ ), was used as the primary antibody to bind to factor VIII. A number of normal plasmas were also studied as negative controls. Primary antibodies were studied in a series of dilutions: seven dilutions for ESH 8 (from $1 / 500$ to $1 / 32,000)$, and six dilutions for human plasma $(1 / 10,1 / 50,1 / 100,1 / 200,1 / 500$ and $1 / 1,000)$.

Coating plates with Recombinate ${ }^{\mathrm{TM}} 5 \mathrm{IU} / \mathrm{ml}$ in carbonate buffer was the minimum factor VIII concentration to give a good linear relationship between monoclonal antibody dilution and OD reading. Also this level of factor VIII for coating gave acceptably low OD values in a buffer blank and normal control plasma. Therefore it was used as the coating concentration in all subsequent tests in this study. The standard series of dilutions used for primary antibody (patient plasma) were $1 / 10,1 / 50,1 / 100,1 / 200,1 / 500$ and 1/1,000. However, the OD reading of $1 / 100$ antibody dilution was chosen to summarise the data. Thus unless specified, all the results in this study were referred to as the results gained with 1/100 plasma dilution. 
Twenty normal plasma samples were studied to establish the normal range of OD reading for the ELISA method. The cut-off value was defined as the mean value of the OD readings of the 20 normal controls plus 3 SD. Any OD reading which was equal to or greater than the cut-off value was considered as a positive ELISA result.

\subsection{Patient Group}

A total of 50 haemophilia A patients ( 33 severe, 5 moderate, 6 mild and 6 acquired cases) were studied by both the ELISA method and the Bethesda assay. Among the adult patients, 10 (including 5 severe) were from Royal Adelaide Hospital Haemophilia Centre, and 12 (including 9 severe) from the Haemophilia Centre of the Alfred Hospital in Victoria. Twenty-four patients showed a detectable inhibitor by Bethesda titre and 26 were undetectable. A summary of the patient data in this study was shown as Table 4.1. The inhibitor titre where positive ranged from 1 to $700 \mathrm{BU} / \mathrm{ml}$ by the Bethesda assay. Among the severe cases $(n=33)$, we studied 20 with and 13 without the intron 22 gene inversion.

\subsection{Different Factor VIII Products}

In addition to Recombinate ${ }^{\mathrm{TM}}$, plasma derived factor VIII AHF (High Purity) (CSL, Australia) and unformulated recombinant factor VIII generously provided by Baxter were also used to coat the micrometre plates, in order to obtain more information about this method. 


\section{TABLE 4.1 Summary of 50 Haemophilia A Patients in the ELISA Study to Detect Factor VIII Antibodies}

Severe, Moderate, and Mild: all refer to inherited patients.

$\mathrm{N} / \mathrm{K}$ : (gene mutation) not known according to current investigations.

N/D: (inhibitor titre) not detectable by the Bethesda assay.

OD Reading: OD reading of $1 / 100$ plasma dilution.

ELISA Result +/-: "+" defined as OD reading = or > cut-off value (0.29);

"-" defined as OD reading $<$ cut-off value. 


\begin{tabular}{|c|c|c|c|c|}
\hline Patient Code & Gene Mutation & $\mathrm{BU} / \mathbf{m l}$ & OD Reading & ELISA Result \\
\hline Severe 1 & intron 22 inversion & 13 & 1.35 & + \\
\hline Severe 2 & intron 22 inversion & 13 & 1.02 & + \\
\hline Severe 3 & intron 22 inversion & 5 & 0.46 & + \\
\hline Severe 4 & intron 22 inversion & 3 & 0.29 & + \\
\hline Severe 5 & intron 22 inversion & 2 & 0.88 & + \\
\hline Severe 6 & intron 22 inversion & 2 & 0.59 & + \\
\hline Severe 7 & intron 22 inversion & 1 & 0.66 & + \\
\hline Severe 8 & intron 22 inversion & 1 & 0.39 & + \\
\hline Severe 9 & intron 22 inversion & N/D & 0.21 & - \\
\hline Severe 10 & intron 22 inversion & N/D & 0.19 & - \\
\hline Severe 11 & intron 22 inversion & N/D & 0.15 & - \\
\hline Severe 12 & intron 22 inversion & N/D & 0.14 & - \\
\hline Severe 13 & intron 22 inversion & $\mathrm{N} / \mathrm{D}$ & 0.12 & - \\
\hline Severe 14 & intron 22 inversion & N/D & 0.12 & - \\
\hline Severe 15 & intron 22 inversion & N/D & 0.10 & - \\
\hline Severe 16 & intron 22 inversion & N/D & 0.09 & - \\
\hline Severe 17 & intron 22 inversion & $\mathrm{N} / \mathrm{D}$ & 0.09 & - \\
\hline Severe 18 & intron 22 inversion & N/D & 0.08 & - \\
\hline Severe 19 & intron 22 inversion & N/D & 0.08 & - \\
\hline Severe 20 & intron 22 inversion & N/D & 0.08 & - \\
\hline Severe 21 & large deletion & 30 & 1.34 & + \\
\hline Severe 22 & large deletion & 23 & 1.41 & + \\
\hline Severe 23 & frame 14 shift & N/D & 0.13 & - \\
\hline Severe 24 & $\mathrm{~N} / \mathrm{K}$ & 4 & 0.72 & + \\
\hline Severe 25 & $\mathrm{~N} / \mathrm{K}$ & 4 & 0.54 & + \\
\hline Severe 26 & $\mathrm{~N} / \mathrm{K}$ & 4 & 0.14 & - \\
\hline Severe 27 & $\mathrm{~N} / \mathrm{K}$ & 1 & 0.82 & + \\
\hline Severe 28 & $\mathrm{~N} / \mathrm{K}$ & N/D & 0.19 & - \\
\hline Severe 29 & $\mathrm{~N} / \mathrm{K}$ & N/D & 0.15 & - \\
\hline Severe 30 & $\mathrm{~N} / \mathrm{K}$ & N/D & 0.12 & - \\
\hline Severe 31 & $\mathrm{~N} / \mathrm{K}$ & N/D & 0.10 & - \\
\hline Severe 32 & $\mathrm{~N} / \mathrm{K}$ & N/D & 0.10 & - \\
\hline Severe 33 & $\mathrm{~N} / \mathrm{K}$ & N/D & 0.10 & - \\
\hline Moderate 34 & point mutation & 2 & 0.44 & + \\
\hline Moderate 35 & point mutation & N/D & 0.09 & - \\
\hline Moderate 36 & point mutation & N/D & 0.07 & - \\
\hline Moderate 37 & $\mathrm{~N} / \mathrm{K}$ & 1 & 0.32 & + \\
\hline Moderate 38 & $\mathrm{~N} / \mathrm{K}$ & N/D & 0.18 & - \\
\hline Mild 39 & point mutation & 700 & 1.42 & + \\
\hline Mild 40 & point mutation & N/D & 0.12 & - \\
\hline Mild 41 & $\mathrm{~N} / \mathrm{K}$ & 23 & 1.54 & + \\
\hline Mild 42 & $\mathrm{~N} / \mathrm{K}$ & $\mathrm{N} / \mathrm{D}$ & 0.17 & - \\
\hline Mild 43 & $\mathrm{~N} / \mathrm{K}$ & $\mathrm{N} / \mathrm{D}$ & 0.15 & - \\
\hline Mild 44 & $\mathrm{~N} / \mathrm{K}$ & N/D & 0.07 & - \\
\hline Acquired 45 & $-\cdots$ & 140 & 1.57 & + \\
\hline Acquired 46 & -- & 15 & 1.29 & + \\
\hline Acquired 47 & --- & 9 & 0.61 & + \\
\hline Acquired 48 & -- & 6 & 0.97 & + \\
\hline Acquired 49 & -- & 3 & 1.36 & + \\
\hline Acquired 50 & -- & 2 & 0.83 & + \\
\hline
\end{tabular}




\section{Results}

\subsection{The Normal Range}

The normal range for the ELISA method was determined by testing 20 normal subjects. The mean OD value for the $1 / 100$ plasma dilution was 0.19 , with minimum 0.13 and maximum 0.26 . The SD was 0.04 , and the $95 \%$ confidence interval of the mean was 0.17 to 0.20 . A cut-off value for positivity was calculated as the mean value plus $3 \mathrm{SD}$, which was 0.29. A positive ELISA result was defined as a result with OD reading equal to or greater than 0.29 ; a negative ELISA result was less than 0.29 . Figure 4.1 shows the binding curve for two normal controls and two patient samples, with respectively negative and positive ELISA results.

\subsection{Comparison of Results Between ELISA and Bethesda Assay}

A group of patients with haemophilia A were tested using the ELISA method. Fifty patients were studied, 24 with inhibitor titres ranging from 1 to $700 \mathrm{BU} / \mathrm{ml}$, and 26 without detectable inhibitors by the Bethesda assay. Figure 4.2 and Table 4.2 summarise the comparison of the results obtained from the two assays. Figure 4.2 shows that all the patients who were negative by the Bethesda assay $(n=26)$ were also negative by ELISA. Therefore there was no evidence for antibodies which bound to factor VIII but did not cause a functional defect. In Table 4.2 more detail is shown for the individual patient groups. Once again, there is a strong correlation between the two assays. Of 24 who were positive by the Bethesda assay, only one was negative by ELISA. The sensitivity of the ELISA as measured against Bethesda assay is $96 \%(23 / 24)$, and specificity $100 \%(26 / 26)$. 


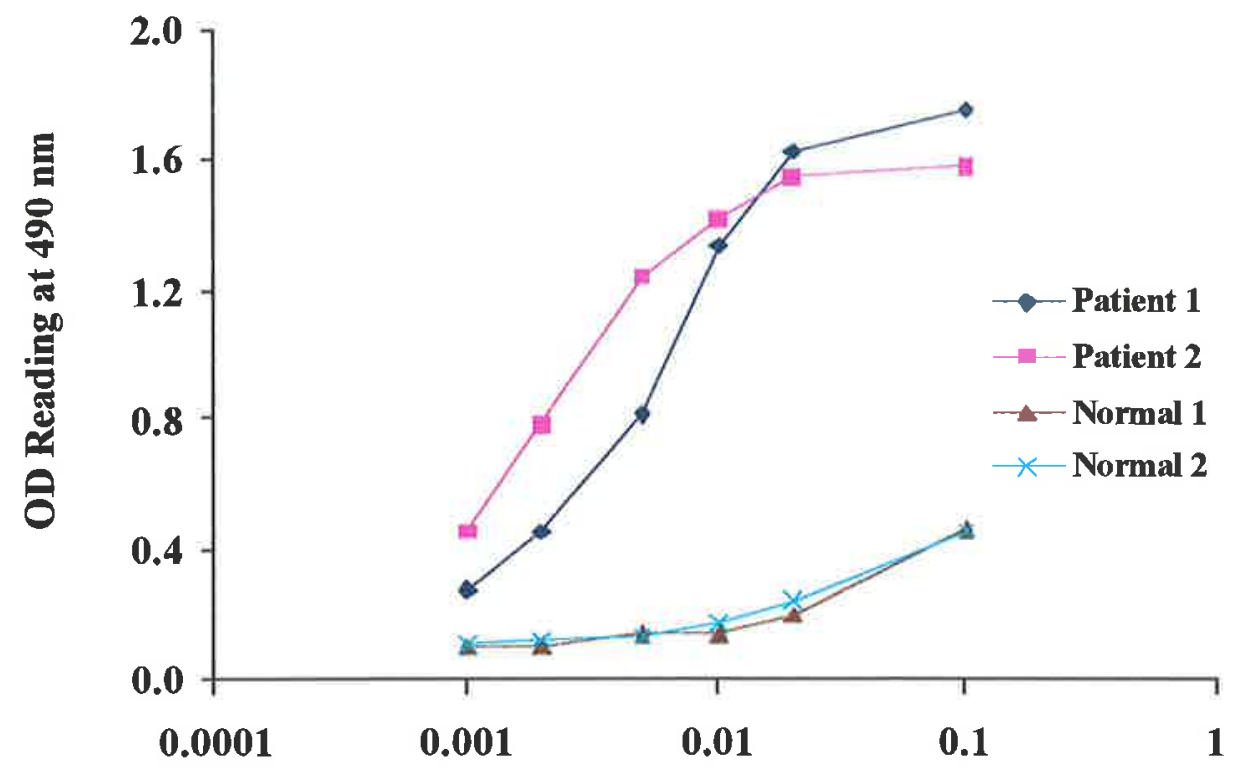

Plasma Dilution

\section{FIGURE 4.1 ELISA Binding Curves for Two Patients and Two Normal Controls}

Both patient and normal plasma samples were tested at six dilutions: $1 / 10$, $1 / 50,1 / 100,1 / 200,1 / 500$ and $1 / 1000$. OD readings at $1 / 100$ dilution were later on chosen to summarise the ELISA results. The two patient samples in this diagram were considered to have a positive ELISA result, while the two normal controls show a negative ELISA result. 


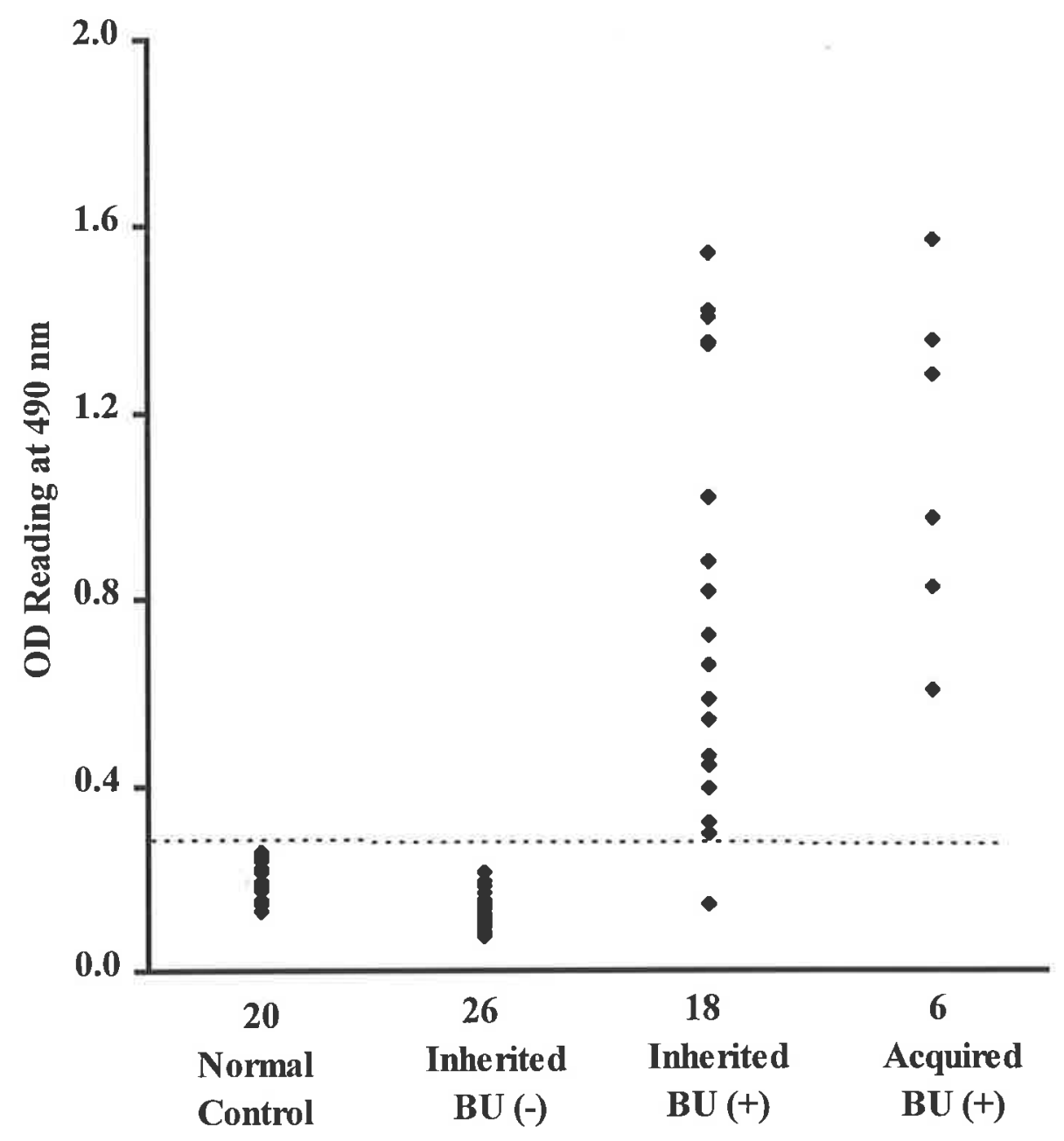

\section{FIGURE 4.2 ELISA Results in Patient Groups with or without Bethesda Titre}

Twenty normal controls were used to determine the positive cut-off for the ELISA (mean+3 SD). Fifty haemophilia A patients were tested, including 18 inherited cases with antibodies detected by Bethesda assay, 26 inherited cases negative by Bethesda assay, and 6 acquired cases. For those cases with a positive Bethesda titre, all acquired and most inherited were also positive by ELISA. For those without detectable antibodies by Bethesda assay, none had a positive ELISA result.

Dashed line: the cut-off OD value (0.29). 
Table 4.2 Comparison of ELISA and Bethesda Results in Detection of Factor VIII Antibodies in 50 Haemophilia A Patients

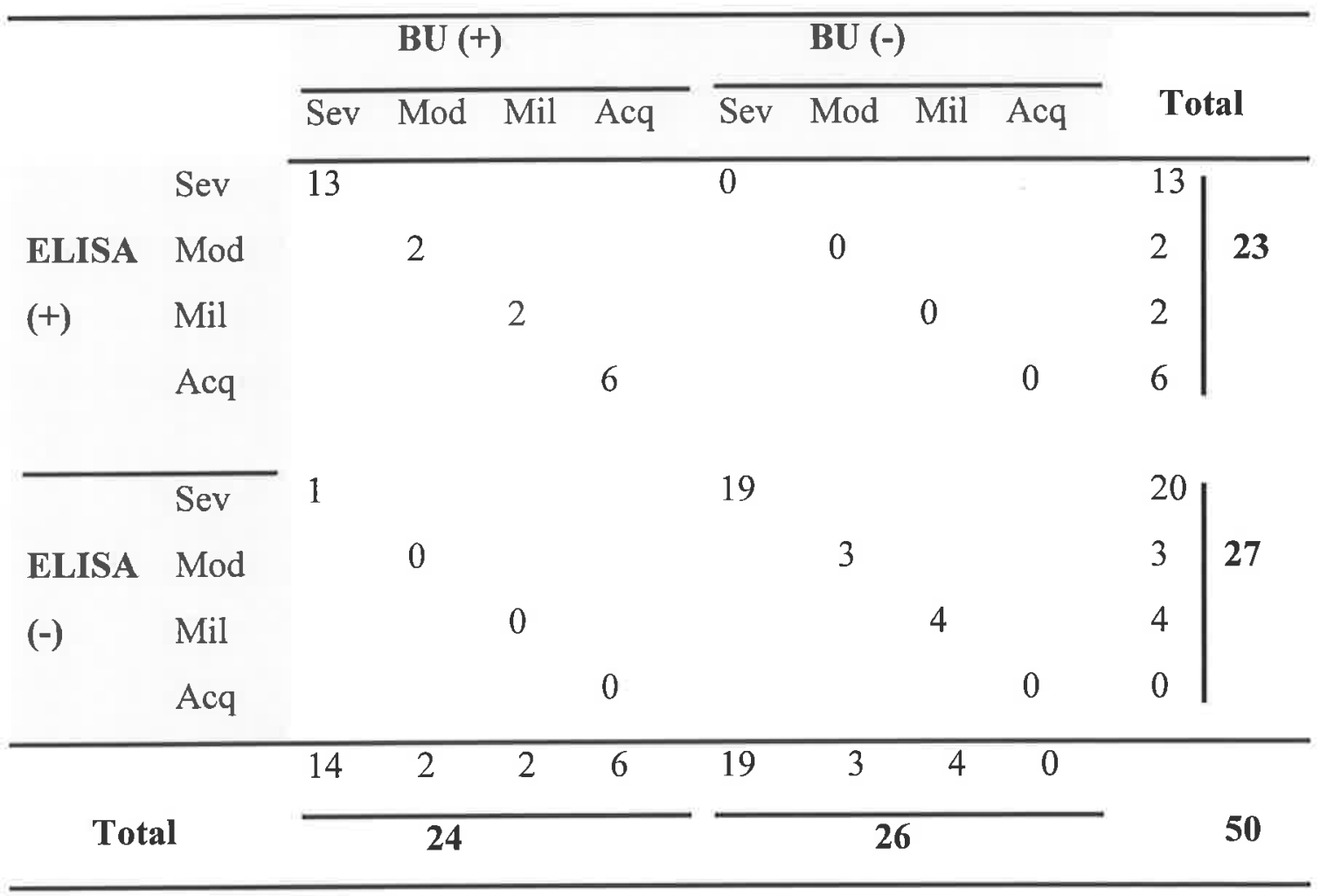

Prevalence of positive ELISA result in patient groups +/- BU:

$\begin{array}{lrlrl}\text { Patients with BU (+): } \quad \mathrm{n}=24 & & \text { Patients with BU (-): } \mathrm{n}=26 \\ \text { ELISA }(+): & 23 / 24=96 \% & & \text { ELISA (+): } \quad 0 / 26=0 \%\end{array}$

Sev, Mod, and Mil: inherited haemophilia A patients with severe, moderate or mild clinical status.

Acq: patients with acquired haemophilia A. 
Figure 4.3 shows the correlation $\left(\mathrm{R}^{2}=0.72 ; \mathrm{P}=0.0001\right)$ between the Bethesda titre and the OD reading on 24 patients with a positive Bethesda result ( 23 with positive ELISA result). There is a reasonable correlation between the two methods.

Patients with severe inherited haemophilia $(n=33)$ were divided into two subgroups: those with intron 22 gene inversion $(n=20)$, the remainder with other gene defects $(n=13)$. As shown in Table 4.3, in our patient subgroup with intron 22 gene inversion, 40\% (8/20) were positive by ELISA, exactly the same result as revealed by the Bethesda assay (40\% positive, $8 / 20$ ). In the subgroup without the inversion, positive results were found in $38 \%$ $(5 / 13)$ and $46 \%(6 / 13)$ of the patients, as determined by ELISA and the Bethesda assay respectively. Again, the two methods for detection of factor VIII antibodies showed a similar sensitivity in both patient subgroups. This was different from the previous study (Vianello et al., 1997), where they showed a much higher percentage $(81 \%, 13 / 16)$ of positive results detected by ELISA in patients with the intron 22 gene inversion. Table 4.3 gives a comparison of our study and the study by Vianello et al. (1997) on the antibody detection in patients with severe haemophilia with and without intron 22 gene inversion.

\subsection{Different Factor VIII Products to Set up the ELISA Method}

A difference between our study and those of Dazzi et al. (1996) and Vianello et al. (1997) was that we used formulated recombinant factor VIII whereas the previous work was done using pure unformulated recombinant factor VIII, to carry out the ELISA. Therefore an attempt was made to set up the ELISA using another source of factor VIII. Both AHF and Baxter unformulated recombinant factor VIII were used to set up the binding curve, and the 


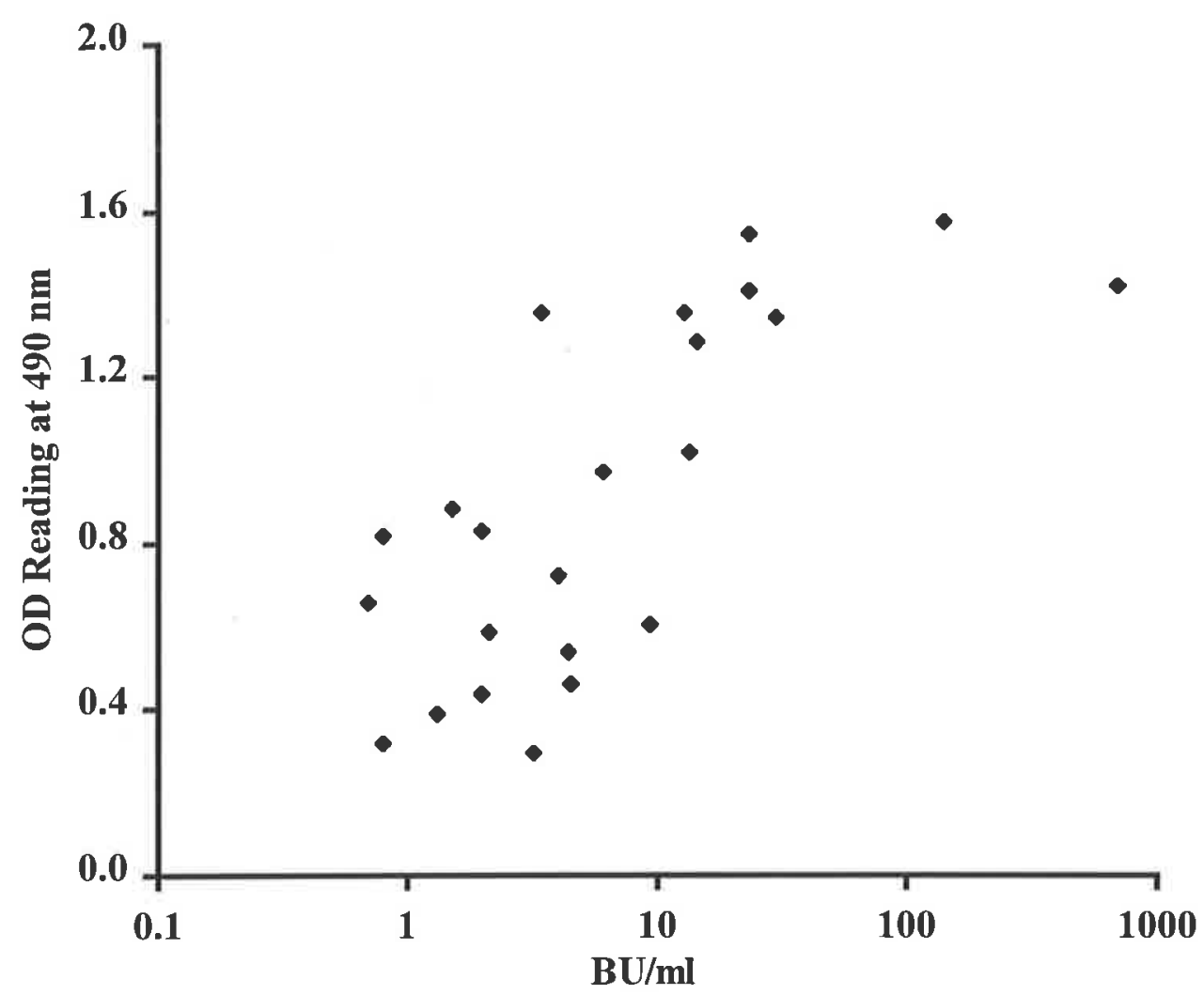

FIGURE 4.3 The Correlation between the Bethesda Titre and the OD Value in ELISA Assay in 23 Patients

Among 50 patient samples studied with both the Bethesda assay and ELISA method to detect factor VIII antibodies, 24 showed a positive Bethesda titre, and 23 out of these patients, also showed a positive ELISA result. The correlation $\left(\mathrm{R}^{2}=0.72 ; \mathrm{P}=0.0001\right)$ was showed as the OD reading (on normal scale) plotted against the Bethesda unit which is on logarithmic scale. 


\section{TABLE 4.3 Comparison of the Two Studies on Factor VIII Antibody Detection in Patients with Severe Haemophilia}

Our Group $(\mathbf{N}=33)$

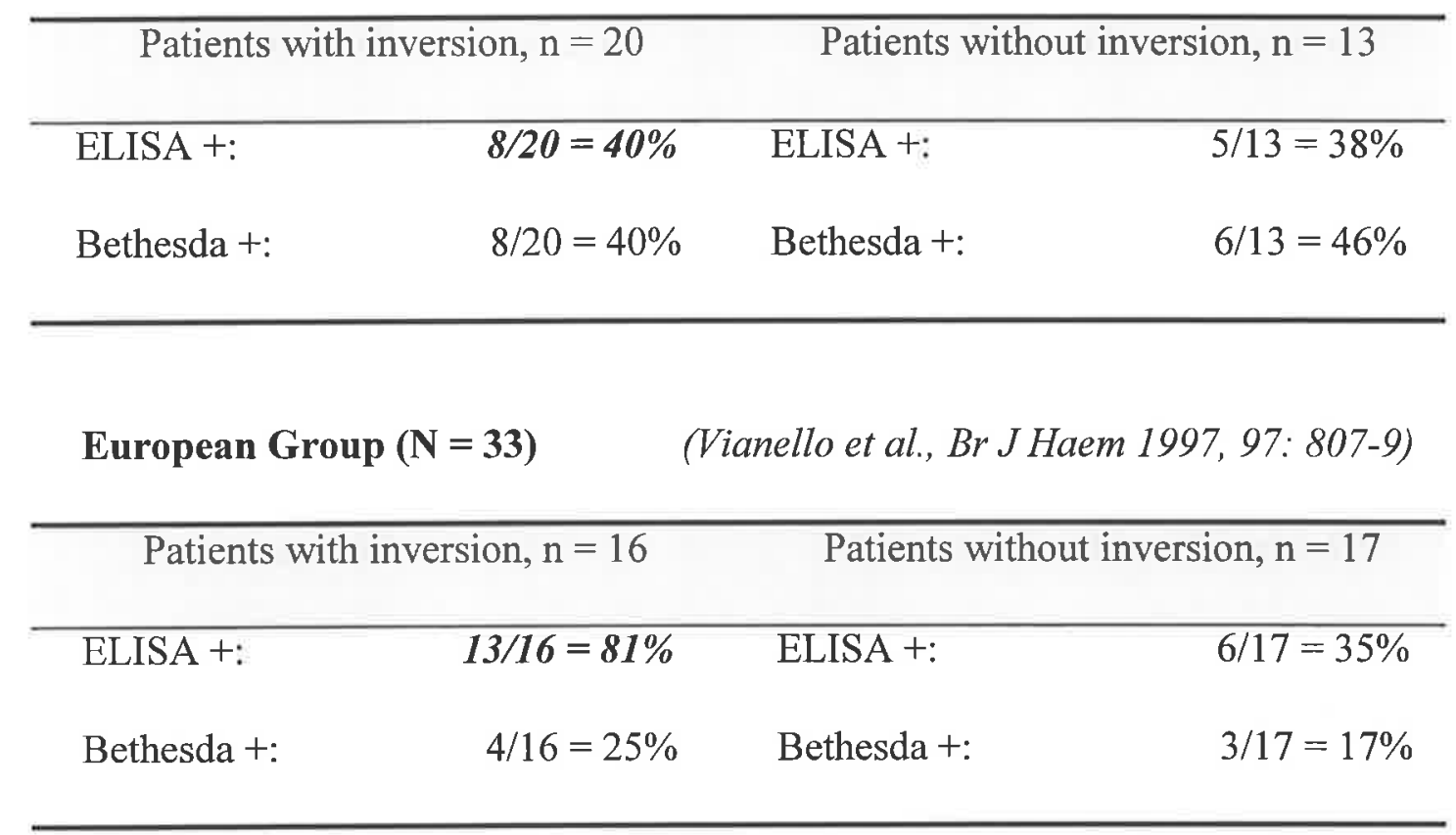

test was then performed on a number of different antibodies including monoclonal antibody, normal plasma, and some patient samples. The results for the three different factor VIII sources using monoclonal antibody ESH 8 are shown in Figure 4.4. The results show that for AHF there was a weaker factor VIII concentration dependent curve compared to use of Recombinant ${ }^{\mathrm{TM}}$ and unformulated recombinant factor VIII (Baxter). Therefore AHF proved to be less suitable for use in this assay and study. The unformulated recombinant factor VIII appeared to perform well and would be suitable for use in future studies. The standard ELISA method using Baxter unformulated factor VIII has been established, with a view to screening our patient plasma samples. This will be helpful to extend our experience with the ELISA method to detect factor VIII antibodies. 


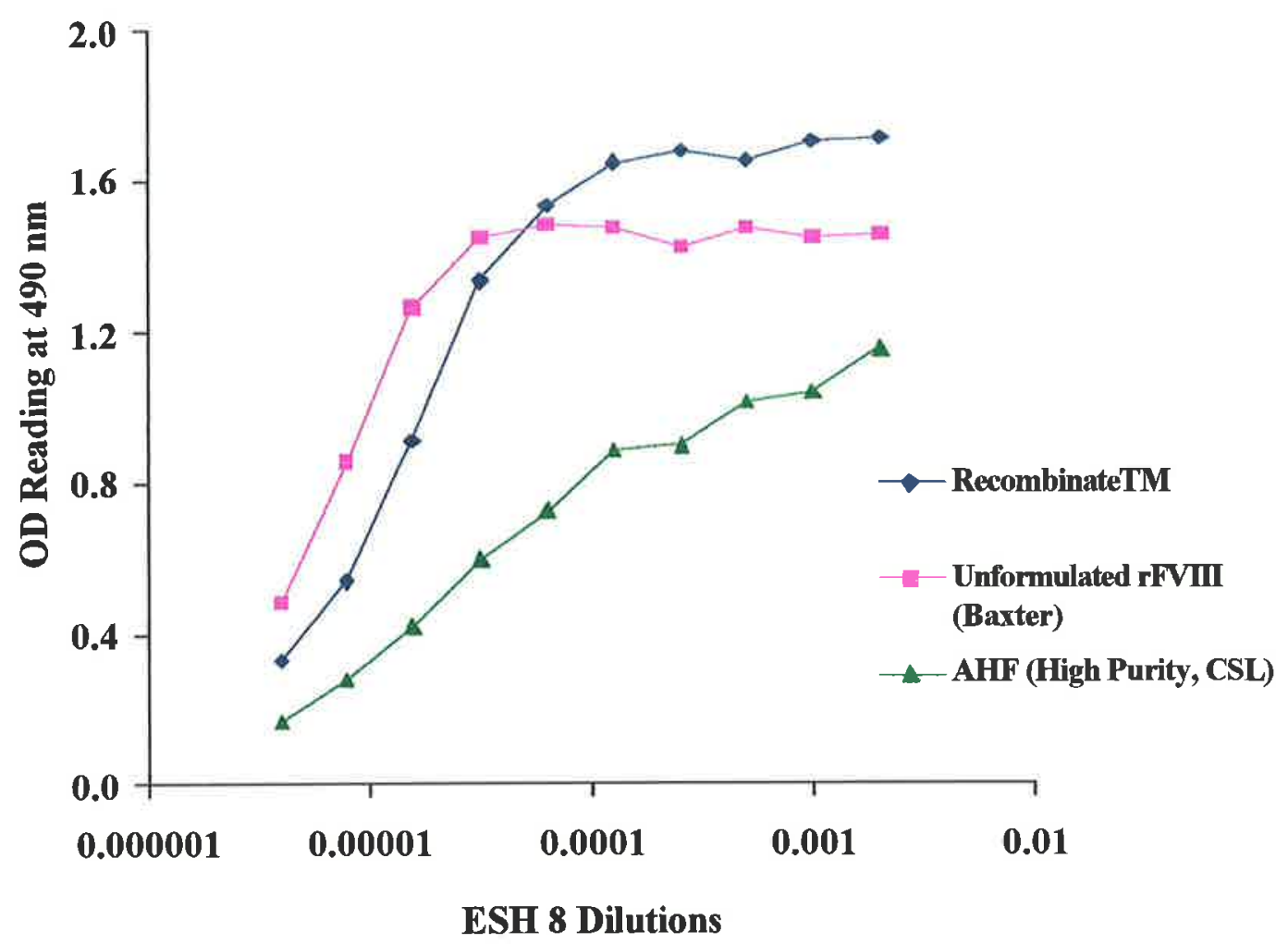

\section{FIGURE 4.4 Three Different Factor VIII Products to Coat the Microtitre Plates in ELISA Method}

This diagram shows the binding curves of a monoclonal anti-factor VIII antibody (ESH 8) to three different products of factor VIII immobilised on microtitre plates. These three products were: plasma-derived factor VIII "AHF (High Purity)" from CSL, formulated recombinant factor VIII "Recombinate ${ }^{\mathrm{TM}}$ " from Baxter, and unformulated recombinant factor VIII also from Baxter. The factor VIII concentration used to coat the plates was 5 $\mathrm{IU} / \mathrm{ml}$, the same for all the products. Ten dilutions (from $1 / 500$ to $1 / 256,000$ ) of ESH 8 were tested. 


\section{Chapter Discussion}

The possible alteration in the structure of the factor VIII molecule during the manufacturing process may cause a change in its antigenicity, thus inhibitors may develop as an immune response to it. In addition, it is suggested that the gene defect which leads to production of an abnormal factor VIII, is also responsible for the induction of the immune response towards a foreign factor VIII in those patients. Therefore, it is considered that the sequences these antibodies target may involve both functional (coagulant) and nonfunctional (non-coagulant) portions of the factor VIII molecule (Gilles et al., 1993; Hoyer, 1995). Thus, depending on the epitope, factor VIII antibodies may be or may be not capable of neutralising FVIII:C activity.

Measurement of factor VIII antibodies is routinely performed by the Bethesda assay which detects only antibodies that inhibit function. This assay has been functioning well in the treatment and clinical evaluation of the haemophilia patients with inhibitors. However, non-inhibitory antibodies may nevertheless theoretically affect the biological half-life of infused factor VIII and be clinically relevant (Regnault and Stoltz, 1994; White, 1994). Therefore a method which can detect binding to both functional and non-functional epitopes could possibly provide useful information. For this reason, immunoassays that are based on measurement of the binding between the antigen and antibody rather than the measurement of physiological function have been adopted by some researchers to explore the detection of factor VIII antibodies (Dazzi et al., 1996; Scandella et al., 1998). A future possible advantage is that some of those immunoassays (such as ELISA, or 
immunoprecipitation) could be more sensitive than the Bethesda assay for detection of antibodies, and may eventually prove to be clinically useful.

To assess and verify the presence of both inhibitory and non-inhibitory factor VIII antibodies, an ELISA method using recombinant factor VIII Recombinate ${ }^{\mathrm{TM}}$ as antigen to coat the plates was established. Twenty normal plasma samples were tested to give a normal range for the OD readings. The result for the ELISA method compared with the Bethesda assay on 50 haemophilia patients showed that the ELISA method has a similar sensitivity to the Bethesda assay for the detection of factor VIII antibodies. Of the 24 samples with a positive Bethesda titre, 23 showed a positive ELISA result, while none of the 26 samples with negative Bethesda titre were positive by the ELISA method. Furthermore, it was demonstrated that there is a weak but definite correlation $\left(\mathrm{R}^{2}=0.72\right.$; $\mathrm{P}=0.0001$ ) between the optical density in the ELISA assay and the Bethesda titre, when the 26 pairs of OD readings (linear scale) were plotted against the inhibitor titres in $\mathrm{BU} / \mathrm{ml}$ (logarithmic scale).

In our study, the detection of the presence of an inhibitor by the ELISA method in patients who have an inhibitor by the Bethesda assay may reflect binding of antibodies to functional sites only. However, in some or all patients it may also reflect additional binding to nonfunctional sites.

The results by the ELISA method were also evaluated in the 33 patients who had severe haemophilia as a separate group. In the 20 patients who had the intron 22 inversion mutation, eight were positive by Bethesda assay and all were also positive by ELISA. In 
the 13 patients with severe haemophilia but without the intron 22 inversion mutation, six were positive by Bethesda assay and all but one was also positive by ELISA. Such results confirmed again the correlation between the two methods for detection of inhibitors. Our results for inhibitor prevalence in patients with the inversion mutation $(40 \%$ by both ELISA and Bethesda methods) agree with these found in the haemophilia A website HAMSTeRS data base, where $35-40 \%$ of patients with the inversion mutation had inhibitors (Tuddenham and McVey, 1998). In our study, the similar sensitivity of the ELISA method compared with the Bethesda assay, was different from some studies by an European group (Dazzi et al., 1996; Vianello et al., 1997). Their conclusion was that the ELISA method revealed a higher prevalence of inhibitors in those patients with a negative Bethesda titre.

It is of interest that our results differ from these in previous studies (Dazzi et al., 1996; Vianello et al., 1997). We did not confirm their findings that some patients had inhibitors that were positive by ELISA but were negative by Bethesda assays. One may argue that such difference could be ascribed to different cut-off values for "positivity" by ELISA in the two studies. However, this is not likely to be the source of bias given the actual data. The cut-off OD value used in our study was 0.29 , which was calculated from the mean value of the 20 normal controls plus $3 \mathrm{SD}$. Even if we used the maximum OD value from the control group as the cut-off value (the European group used the highest value of six normal controls), it would not change the result of "positive" or "negative" in any of our 50 patients. Nevertheless, there could be a number of possible reasons count for the differences found in our study and the European study. They are, firstly, methodological factors, which may be very important. The most likely factor in our study is the source of factor VIII used to coat plates. Different factor VIII products may have different epitopes 
exposed on the microtitre plates. The factor VIII used by Dazzi et al. (1996) and Vianello et al. (1997) was unformulated recombinant factor VIII supplied by Baxter. For our study, we used the formulated Baxter product. Further work with the unformulated product is necessary to determine if these products have different antigenic properties in the ELISA method. Secondly, one has to consider whether there might be differences in the patient population, particularly in the prevalence of the different causative mutations. However, it is likely that our population is very similar to that examined in the European studies. The third possibility is that there may be environmental factors that are different in the Australian population. This could include the type of replacement therapy that the patients received. In Australia, AHF made by CSL is used exclusively for those patients treated with a plasma-derived product. In other countries, e.g., in Europe, other products from a variety of manufacturing methods are used. It is likely that the factor VIII concentrates produced by different manufacturing technique lead to the exposure of different epitopes on the factor VIII molecule. It is well described that some products have proved to be excessively antigenic (Rosendaal et al., 1993; Peerlink et al., 1997; Vermylen, 1998). Two products have been withdrawn from use after the development of inhibitor in a number of cases who had been previously treated with other products without inhibit formation (Rosendaal et al., 1993; Peerlink et al., 1997).

The sensitivity and reliability of our ELISA method still needs further confirmation, using at least one other source of factor VIII to coat the plates. For this reason, we have very recently optimised the ELISA for use with unformulated recombinant factor VIII to coat the plates. Initial experiments demonstrated that unformulated recombinant factor VIII 
will be suitable for use in the ELISA method. In the future, it is planned to retest our group of patients using the unformulated factor VIII to detect antibody binding.

It is worth mentioning that there was one case of an unexpected discrepancy between methods, with a negative result by ELISA and the presence of inhibitors by the Bethesda assay. The inhibitor titre was $4 \mathrm{BU} / \mathrm{ml}$ (patient "Severe 26", Table 4.1). Such a discrepancy was also reported in Vianello's study (Vianello et al., 1997). This suggested that immunoassays such as ELISA, cannot detect all types of anti-factor VIII antibodies. This might be related to the conformational changes of the epitopes on the factor VIII molecule during production, immobilising or testing procedures, which then become unrecognisable to some antibodies. Another explanation may be related to the affinity/avidity of the antigen-antibody complex reflected in the ELISA method. The inhibitors in this case may have had a loose-binding to the immobilised factor VIII antigen on the microtitre plate, so as to give a low OD reading which was not comparable to the degree of inhibition of FVIII:C as detected by the Bethesda method. It will be of interest to detect whether this patient's inhibitor is type I or type II.

\section{Chapter Summary}

In summary, the establishment of immunoassays such as ELISA provides an alternative way to examine the immune response to factor VIII in haemophilia A patients, in addition to the traditional coagulation tests.

- An ELISA method using recombinant factor VIII to coat the microtitre plates has been established to detect anti-factor VIII antibodies. The cut-off OD value 0.29 in $1 / 100$ plasma sample dilution was used to define a positive or negative ELISA result. 
- The ELISA was run in parallel with the Bethesda assay on 50 haemophilia A patients and showed a similar sensitivity for detection of factor VIII antibodies. There was a correlation between the Bethesda titre and the OD found in ELISA tests. In studies reported by others there has been a lack of correlation, with many patients who were negative by functional assay testing as positive by ELISA.

- The discrepancy between our study and other studies may reflect methodological differences. It is very important to repeat our study using different sources of factor VIII to coat the plates. The discrepancy may also be caused by the differences in the patient population. In particular it may reflect the type of factor VIII used to treat our patients. 


\section{CHAPTER FIVE}

\section{EPITOPE DETECTION ON FACTOR VIII PEPTIDE}

\section{Introduction}

Factor VIII is a high-molecular-weight glycoprotein composed of three different types of domains arranged in the order $\mathrm{NH}_{2}-\mathrm{A} 1-\mathrm{A} 2-\mathrm{B}-\mathrm{A} 3-\mathrm{C} 1-\mathrm{C} 2-\mathrm{COOH}$, as deduced from the cDNA sequence (Wood et al., 1984). It circulates in the plasma as a heterodimer of a heavy chain (A1-A2-B) and a light chain (A3-C1-C2) linked by a metal ion bridge. The anti-factor VIII immune response is polyclonal, and antibodies are directed towards almost any region or domain (Gilles et al., 1997).

Initial epitope mapping studies utilising characteristic thrombin cleavage fragments of factor VIII in immunoblots noted that epitopes resided on both the heavy and light chains (Fulcher et al., 1985; Fulcher et al., 1987). Approximately one-third of patients produced inhibitors which bound to the heavy chain, one-third bound to the light chain and one-third bound to both the heavy and light chains. Bacterial expression clones of fragments of the factor VIII gene, either intact or with engineered deletions, were used to localise the epitopes further down to the A2 and the C2 domains (Scandella et al., 1988; Scandella et al., 1989). When the variable recombinant fragments were tested for inhibitor antibody reactivity on immunoblots, most inhibitor-containing plasma was strongly reactive with epitopes mapping either to residues $379-538$ of the heavy chain, residues $2178-2332$ of the light chain, or both. In addition, other less commonly reactive epitopes were localised to the amino terminal end of the light chain within the $\mathrm{A} 3$ or $\mathrm{C} 2$ domains (Scandella et al., 1988). Epitopes in the A1 and B domains have rarely been reported. 
As discussed in Chapter 3, the different kinetics of inactivation of factor VIII seen for inhibitors may be a consequence of the binding-site on the molecule. Also, other features of the inhibitor response, e.g., responder group, cross-reactivity to porcine factor VIII, high versus low titre, may be related to the specific epitopes.

To explore these concepts further I undertook development work to study epitopes on the factor VIII molecule. Therefore the specific chapter aims were:

1) To establish a method to produce factor VIII heavy and light chain fragments, using thrombin to cleave the molecule and immunoblotting to detect antibody binding.

2) To investigate the use of a bacterial (E.Coli) expression system to produce recombinant factor VIII fragments (in particular, domain A1), with a view to using this fragment to screen factor VIII antibodies (including monoclonal antibody and patient antibody).

\section{Methods}

The generation of thrombin-cleaved factor VIII fragments followed by immunoblotting was one of these earlier methods used to locate epitopes (Fulcher et al., 1985; Fulcher et al., 1987). This approach allows binding to heavy and light chain specifically to be determined, as well as binding to the smaller products of the activation and inactivation steps. The method used is described in Chapter 2, section 3.3.2.

For the majority of these experiments the source of factor VIII was Recombinate ${ }^{\mathrm{TM}}$ (Baxter), the recombinant therapeutic product which contains a number of stabilisers including albumin at $12.5 \mathrm{mg} / \mathrm{ml}$. Unformulated recombinant factor VIII (also from Baxter), without albumin, was available towards the end of the project. The concentration 
of FVIII and thrombin used were similar to those previously described. The primary monoclonal antibodies used included MAS 530 and MAS 531 at 1/100. These antibodies had no detectable activity in a Bethesda assay and therefore $\mathrm{BU} / \mathrm{ml}$ can not be given (results not shown). ESH 8 was also studied, at dilution of 1/1000. This antibody, which is directed against a light chain/C2 epitope, showed inhibitor activity in the Bethesda assay (approximately 3,000 BU/ml, results determined by routine laboratory) and ELISA method (Chapter 4). In patient studies, IgG from two patients with 7 and $43 \mathrm{BU} / \mathrm{ml}$ activity was used, and diluted to $1 / 100$ for the immunoblotting procedure. The secondary antibodies (with HRP linkage for the ECL detection step) were anti-mouse IgG for the monoclonal antibodies and anti-human IgG for use with the patient studies. During the immunoblotting procedure the gel was cut into strips as required to allow the appropriate incubations.

My attempt to use a bacterial expression system to produce the A1 domain was unsuccessful. Nevertheless, considerable time was spent working on each phase of the system to produce recombinant protein. The methods used are described here, and the problem arising at various stages are given in the Results section. The procedure followed for expression vector cloning basically included: PCR to make the target gene, insertion of target gene into the vector, transformation of the vector to $E$. Coli cells, screening the transformed cell clones, induction of target gene expression, and protein purification. Methods were described in Chapter 2, Section 3.3.1. 


\section{Results}

\subsection{Thrombin-Cleaved Fragments of Recombinant Factor VIII}

Recombinate $^{\mathrm{TM}}$ was incubated with thrombin in a time course study (incubation time: 0 min, $10 \mathrm{~min}$, and $20 \mathrm{~min}$ ) and then incubated with either monoclonal antibody ESH 8 (antiC2 domain of light chain) or MAS 530 (anti-heavy chain), as shown in Figure 5.1. There were two bands shown at the size of heavy chain fragments $(\sim 50 \mathrm{KD}, 45 \mathrm{KD})$ as detected by MAS 530, in lanes loaded with thrombin cleavage samples (10 min [lane 8] and $20 \mathrm{~min}$ [lane 7] incubation samples). There were no 45-50 KD bands found with the uncleaved rFVIII sample lane (lane 9). Probing with the monoclonal antibody ESH 8 did not show any binding results. Cross reaction between the antibodies and albumin (at $61 \mathrm{KD}$ ) caused significant interference.

In the next experiment monoclonal antibodies MAS 530 (anti-heavy chain) and MAS 531 (anti-light chain) were used. As shown in Figure 5.2, two small fragments ( $\sim 50 \mathrm{KD})$ similar to those seen in the previous experiment, were demonstrated by MAS 530 to bind to the heavy chain product. This again occurred only in the thrombin-cleaved rFVIII sample lanes (lanes 2 and 5). Anti-light chain antibody MAS 531 did not show any specific binding to factor VIII fragments.

Based on the positive western blot result of MAS 530 binding to thrombin cleaved rFVIII fragments, we further investigated this detection method with some of the samples from our inhibitor patients. Figure 5.3 shows the results obtained from $\operatorname{IgG}$ concentrates of two of our patients with severe inherited haemophilia (Case 3 and Case 5 in Chapter 3). As before, MAS 530 was used for a positive control (binding with two small fragments as 


\section{FIGURE 5.1 Time Course of Thrombin Cleavage of Recombinant Factor VIII Detected by Western Blot, Using Two Monoclonal Antibodies}

Column M: molecular weight markers (KD).

Column B: blank (loading buffer only).

Column 2-5: detection with monoclonal antibody ESH 8.

Column 7-10: detection with monoclonal antibody MAS 530.

Column $2 \&$ 7: thrombin incubated with rFVIII for $20 \mathrm{~min}$.

Column $3 \&$ 8: thrombin incubated with $\mathrm{rFVIII}$ for $10 \mathrm{~min}$.

Column 4 \& 9: thrombin incubated with rFVIII for zero $\mathrm{min}$.

Column 5 \& 10: albumin control, for identification of this contaminant by its molecular weight.

The arrows on the right hand side indicate the expected products after heavy chain breakdown (lanes $7 \& 8$ ) which can be detected by MAS 530, i.e., the 50 and $45 \mathrm{KD}$ fragments. 


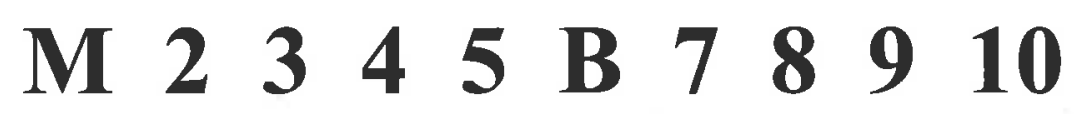

187

118

85

$61-$

50

38
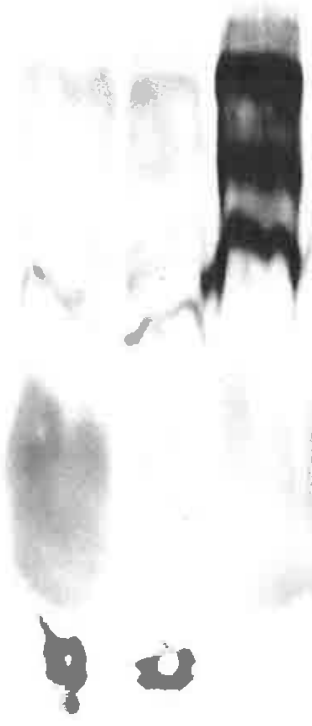

1 


\section{FIGURE 5.2 Western Blot for Thrombin-Cleaved Recombinant Factor VIII, Using Both Anti-heavy Chain (MAS 530) and Anti- Light Chain (MAS 531) Monoclonal Antibodies}

Column M: molecular weight markers (KD).

Column B: blank (loading buffer only).

Columns 2-6: detection with anti-heavy chain monoclonal antibody MAS 530.

Columns 8-10: detection with anti-light chain monoclonal antibody MAS 531.

Columns $2 \& 3,5 \& 6$, and $8 \& 9$ : each pair loaded with thrombin-cleaved rFVIII (15 min incubation) and zero control (rFVIII plus $\mathrm{H}_{2} \mathrm{O}$ instead of thrombin) respectively. Dose effect was studied with MAS 530 detection by reducing to half the amount loaded in lane paires $2 \& 3$.

Column $4 \&$ 10: albumin control, for identification of this contamination by its molecular weight.

The arrows on the right hand side indicate the expected products after heavy chain breakdown (in lanes $2 \& 5$ ), which can be detected by MAS 530 as described before. However, there was no binding for MAS 531 (lanes 8 \& 9) to any potential factor VIII fragments (including $80 \mathrm{KD}$ and $73 \mathrm{KD}$ light chain fragments), except for the albumin contamination. 


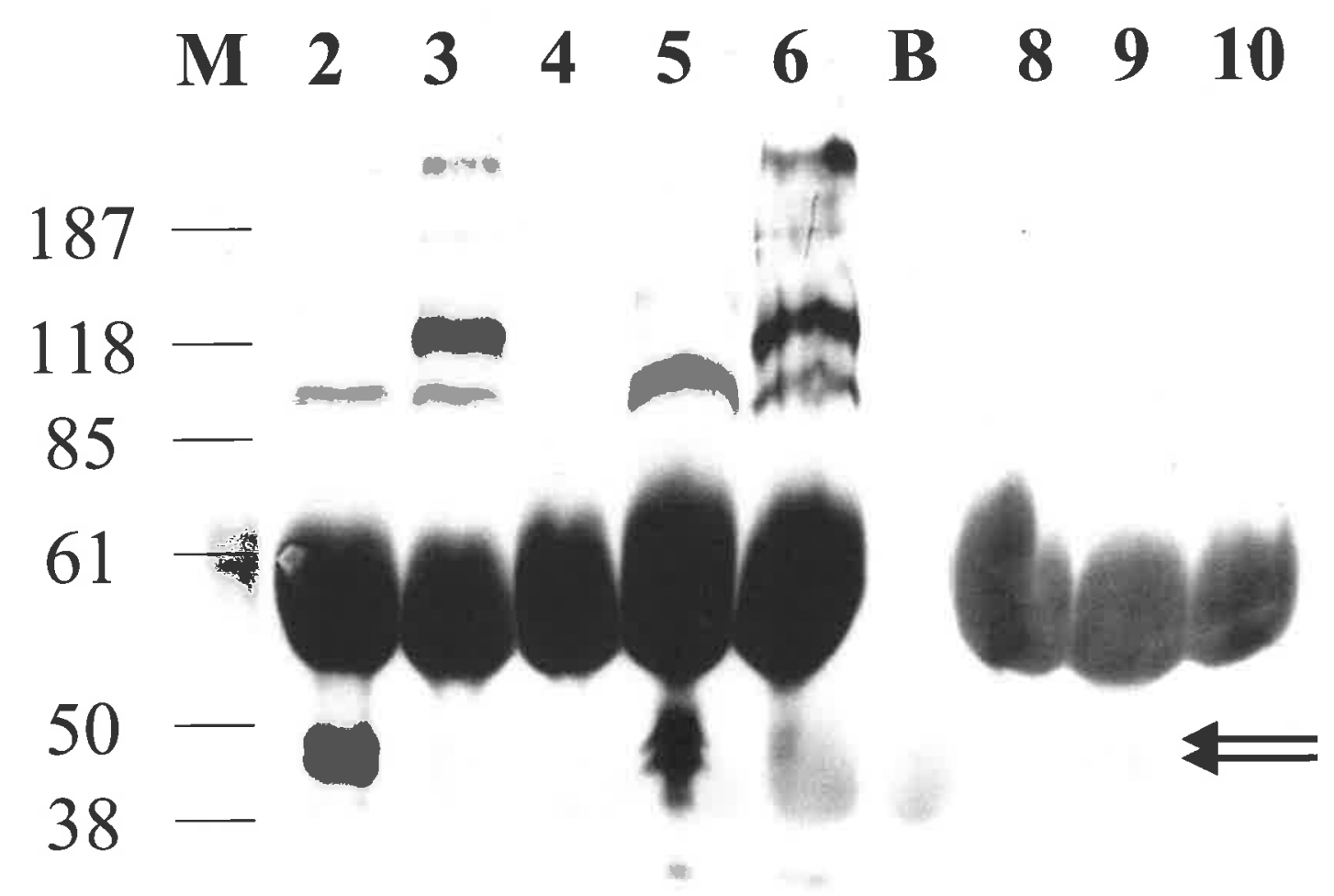




\section{FIGURE 5.3 Western Blot for Thrombin-Cleaved Recombinant Factor VIII, Using Monoclonal Antibody (MAS 530) and Human IgG Concentrates}

Column M: molecular weight markers (KD).

Column 2, 3 \& 4: loaded with zero control rFVIII (rFVIII plus $\mathrm{H}_{2} \mathrm{O}$ instead of thrombin), thrombin-cleaved rFVIII (15 min incubation), and albumin control respectively. Probed with monoclonal antibody MAS 530.

Columns 5, $6 \& 7$, and $8,9 \& 10$ : the same rFVIII order as in lanes $2,3 \& 4$, but probed with IgG concentrates from two patients with inhibitors (Case $3 \mathrm{Hu}$ and Case 5 Lo).

The arrows on the right hand side indicate the expected bindings of the heavy chain fragments after cleavage (lane 3) to MAS 530, as shown before. However, there were only background signals in lanes 5-7 probed by IgG from Case 3, and a totally blank gel in lanes 8-10 probed by IgG from Case 5 . 


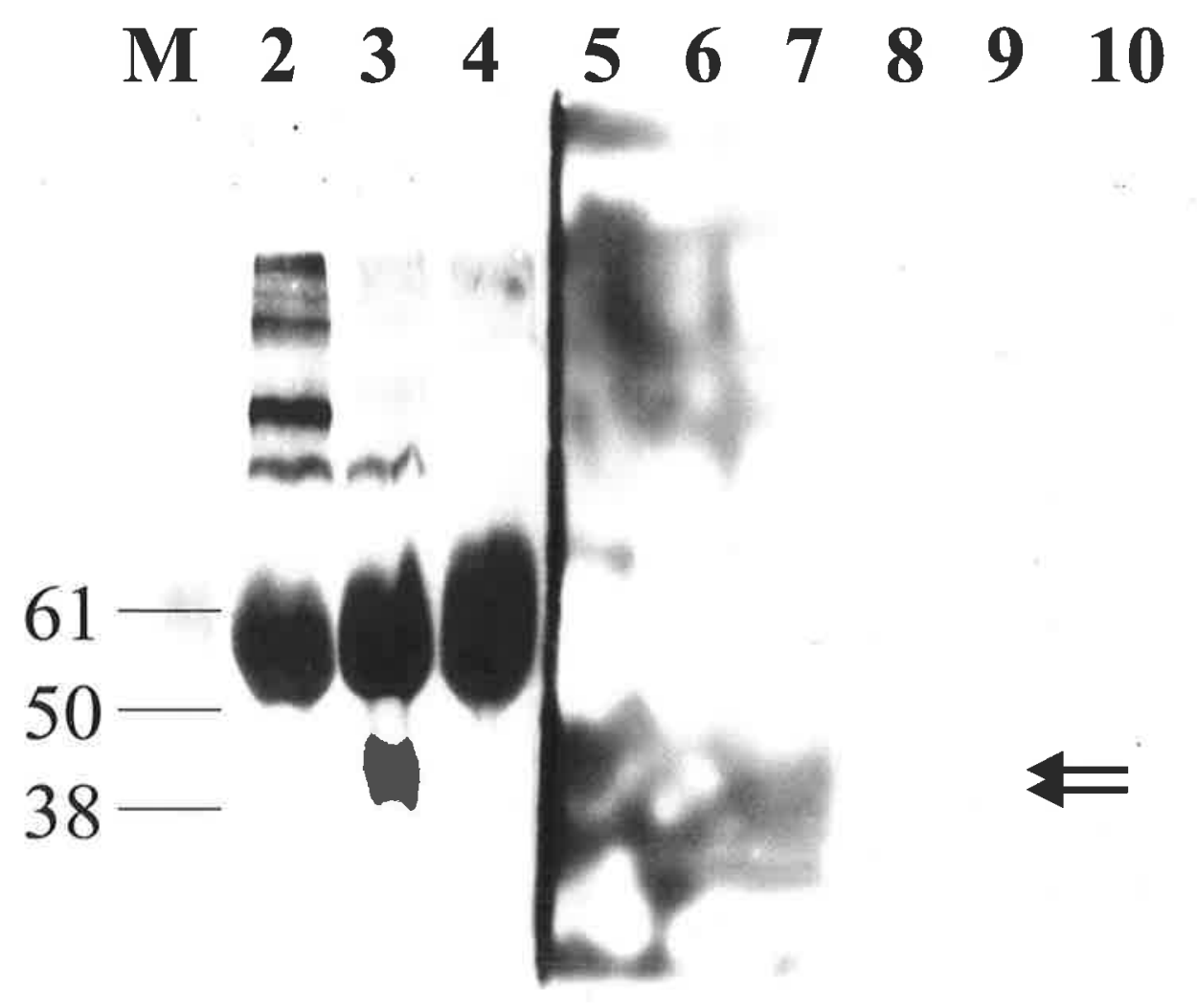


shown in lane 3, which was a thrombin-cleaved rFVIII sample lane). However, no binding could be demonstrated for either patients' IgG: one showed a high background signal, while the other gave no signal.

Towards the end of this study, we were able to use unformulated rFVIII (a gift generously provided by Baxter) to set up western blot analysis of thrombin-activated rVIII. Therefore, the interference by albumin in formulated rFVIII was avoided. The result is shown in Figure 5.4. There was binding to bands similar to those in previous studies detected by MAS 530 in lanes 3 and 5, which were loaded with different amounts of thrombin-cleaved unformulated rFVIII (15 min incubation). Again, monoclonal ESH 8 did not show any evidence of binding.

\subsection{Bacterial Vector Expression of Factor VIII Fragment A1}

The following shows results of attempts to produce recombinant A1 fragment. Attempts to produce heavy chain, $\mathrm{A} 1 / \mathrm{A} 2$, and $\mathrm{A} 2$ fragments were also performed during the research period of this project, with none of them, however, achieving any successful result. This work was performed in conjunction with a current research project carried out in the Molecular Pathology Division at IMVS. The method and results following show parts of the work I undertook to produce the A1 domain. The principle used to produce the A1 domain can be applied to other segments of factor VIII.

Briefly, the main work with recombinant DNA technology to produce protein fragments comprised: a) PCR to make a target gene. b) Insertion, transformation, and screening for the specific cell colonies that contained the inserted vector and target gene. c) induction 


\section{FIGURE 5.4 Watern Blot for Thrombin-Cleaved Unformulated Recombinant Factor VIII (Baxter), Using Monoclonal Antibodies MAS 530 and ESH 8}

Column M: molecular weight markers (KD).

Column B: blank.

Column 2 \& 3, 4 \& 5: loaded with zero control rFVIII (unformulated rFVIII plus $\mathrm{H}_{2} \mathrm{O}$ instead of thrombin), thrombin-cleaved unformulated rFVIII (15 min incubation), in the two lanes respectively. The samples loaded in lanes $2 \& 3$ were double the amount loaded in lanes $4 \& 5$. All probed by monoclonal antibody MAS 530.

Columns $7 \& 8,9 \& 10$ : duplicates of the lanes $2 \& 3,4 \& 5$, but probed by monoclonal antibody ESH 8.

The arrows on the right hand side indicate the expected bindings of the heavy chain fragments of the unformulated factor VIII after cleavage (lane 3 and 5) to MAS 530, as the same seen before. Again nothing came up with the monoclonal antibody ESH 8. With this product there is no interfering band of albumin. 
$\begin{array}{llllllllll}\text { M } & 2 & 3 & 4 & 5 & \text { B } & 7 & 8 & 9 & 10\end{array}$

$61-$

$50-$

$38-2$

$\rightleftarrows$ 
and purification of the protein. Figure 5.5 shows the evidence of a successful PCR result for A1 gene. Gel electrophoresis showed a clear band of the right size for the A1 gene $(\sim 1.3 \mathrm{~kb})$, while there was no band in the non-cDNA control lane.

After purification of the PCR products, digested by BamH I restriction enzyme, ligation was set up to insert the A1 gene into the vector pGEX-2TK. Then the vector was introduced into an $E$. Coli competent cell, $\mathrm{DH} 5 \alpha$, and allowed to grow on LB-Amp agar plates at $37^{\circ} \mathrm{C}$. By destroying the cell structure and running the DNA components on the gel, it was possible to select those colonies with a successfully inserted vector gene. With these clones, in addition to a large band which indicated the cell gene itself, there was another band of the right size for the vector gene $(\sim 4.8 \mathrm{~kb})$ plus the A1 gene. As shown by Figure 5.6, among quite a few randomly selected colonies, it appeared only the No. 21 colony demonstrated the inserted vector, with the arrow indicating the extra DNA band of the right size.

Colony 21 (and also 14) were selected and then put into a large culture for protein expression. Figure 5.7 shows the results of induction using IPTG. "GEX" was the vector alone, used as a control, in the induction procedure. After induction, if the cell would express A1 domain successfully, there would be an extra band corresponding to the size which is indicated by the arrow with the short dashed line on Figure 5.7. There was no extra band in lanes of the 1,2 or 3 hour time points of the induction process as compared with that in the zero point sample lane, or compared to the GEX control, at this molecular weight. There was an extra band for the GEX control, at the approximate molecular weight of the vector (solid arrow), expressed after 1 hour induction. 


\section{FIGURE 5.5 PCR Products for the A1 Gene}

Column M: molecular weight markers for DNA $(\mathrm{kb})$, does not show up well on this photograph.

Columns 3, 4 \& 5: with PCR products at the expected molecular weight $(\sim 1.3 \mathrm{~kb}$ for the A1 gene), as indicated by the arrow.

Column C: negative control without cDNA put in prior to the PCR reaction, showing no PCR products. 


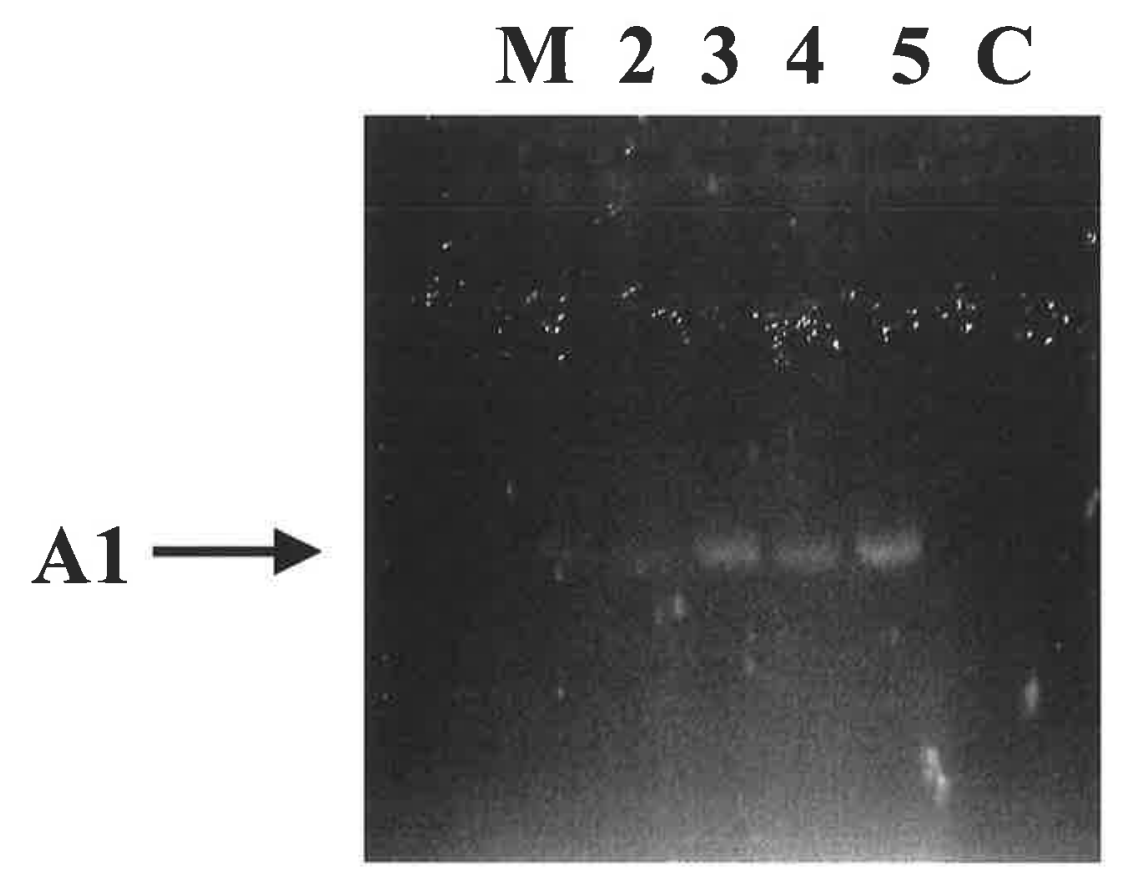


FIGURE 5.6 Colony Screening for the Insertion of A1 Gene into the Vector

Column M: molecular weight markers for DNA $(\mathrm{kb})$, does not show up well on this photograph.

Colony 21 , shows a band indicated by the left hand arrow, which suggests incorporation of the $\mathrm{A} 1$ gene into the vector. 


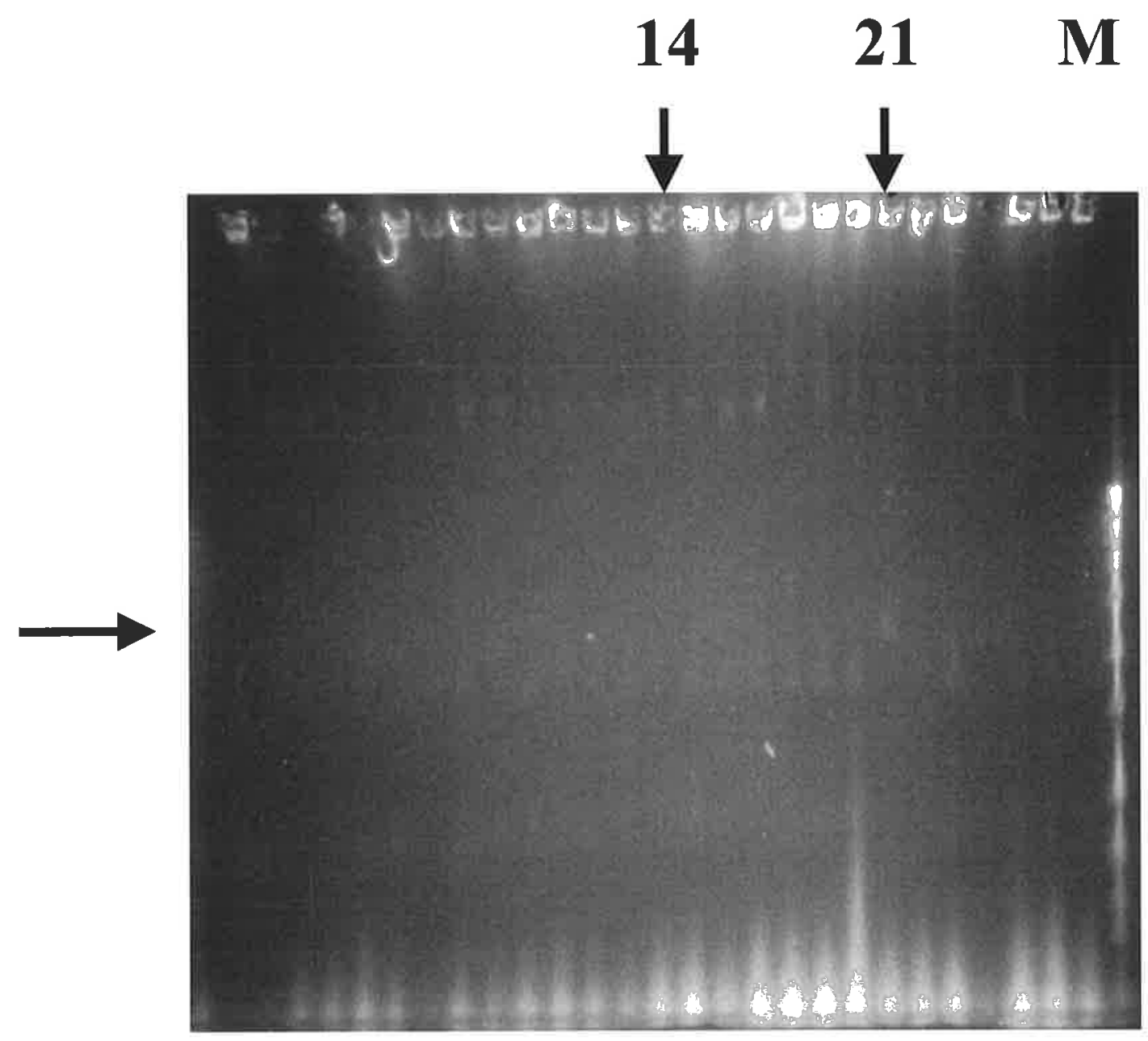




\section{FIGURE 5.7 IPTG Induced Protein Expression by Colony 21}

Coomassie Blue to stain the gel.

Column M: molecular weight markers for protein (KD).

Samples were taken at different time points after adding the IPTG. GEX indicates vector alone, and A1 indicates the samples taken from the transformed culture.

The dashed arrow indicates the molecular weight of the expected A1 peptide.

The solid arrow shows the expected molecular weight of the vector alone. 


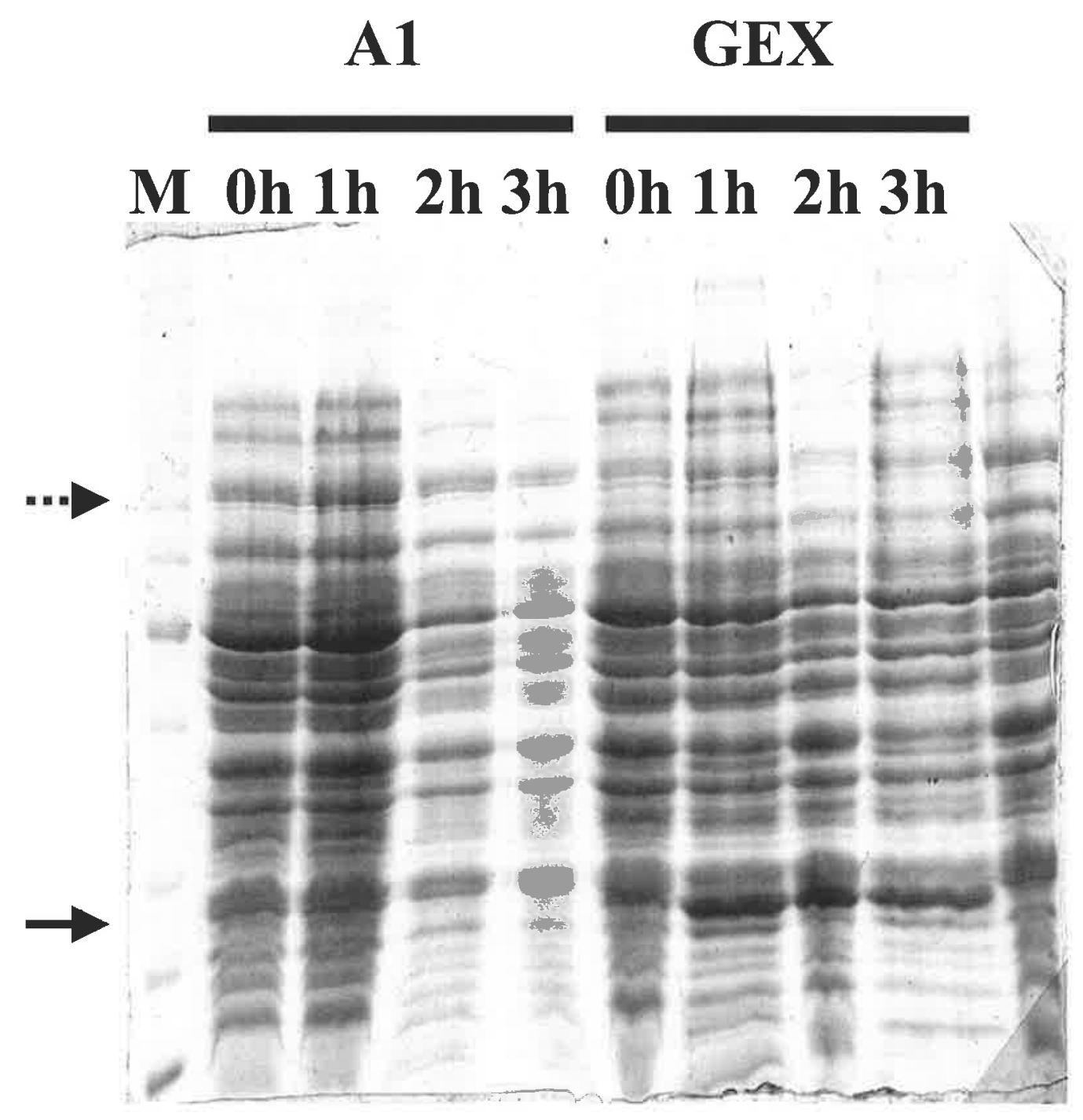




\section{Chapter Discussion}

Thrombin-cleaved recombinant factor VIII fragments, namely, the heavy chain and its activation/inactivation products demonstrated binding with monoclonal anti-heavy chain antibody MAS 530. This was an initial experiment to show that epitopes could be detected. The method was then used in our patients to detect binding of patient inhibitors. It is important to mention that immunoblotting may cause lots of antigenic determinants due to denaturation, and it may therefore not be possible to detect the binding of antibodies to those epitopes (Hoyer, 1991; Hoyer and Scandella, 1994; Scandella, 1996). In addition to immunoblotting, other antibody binding assays such as immunoprecipitation should also be valuable in epitope determination. Also, identification of the clinical relevance of specific epitopes requires an antibody neutralisation assay, in which the same soluble factor VIII peptide as found with binding is tested for its ability to neutralise the inhibitory function of the antibody to FVIII:C (Scandella et al, 1995 b).

The failure in expression of factor VIII fragments in E. Coli cells illustrates the difficulty of this method. More work needs to be done in order to optimise the molecular biological conditions which are required for successfully producing a fragment. Theoretically, once set up, this method can be easily adapted to produce a variety of factor VIII fragments in a range of sizes.

However, we showed evidence of insertion of the vector gene (with A1 gene) into the cell, as the colony screening shown in Figure 5.6. Therefore, we consider the reasons that the A1 protein was not expressed might be: 1) the product is toxic to the cell. 2) the induction 
conditions need to be optimised. 3) the cell line is not reliable, or the cell line is not suitable for the production of A1 domain. In addition, there might had been some A1 protein produced, but it was very unstable or insoluble, so that it was not detected. We have become aware that other research groups working on the factor VIII protein are finding that insect cell lines are superior to $E$. Coli for the production of factor VIII and fragments of factor VIII. Since my project, the use of insect cell lines has been introduced in our laboratory.

In this study thrombin cleaved fragments of recombinant factor VIII protein were generated with the aim of determining heavy and light chain specificity of inhibitors. Whilst successful with a monoclonal antibody model the approach was unsuccessful for the study of IgG purified from patient plasma. This is likely due to insufficient sensitivity of the system for the relatively low levels of anti-factor VIII antibody tested $(\sim 7-40 \mathrm{BU} / \mathrm{ml}$ from patient IgG concentrates). Further directions for this work could include the use of a more sensitive detection system in the immunoblotting method (e.g., streptavidin-biotin or $I^{125}$ labelled secondary anti-human antibody). Also, the production of recombinant factor VIII fragments would allow more precise localisation of epitopes.

The mechanisms by which factor VIII inhibitors inactivate FVIII:C are variable. At least one mechanism, which is relevant to inhibitors with light chain specificity (C2 domain), is interference with normal factor VIII-phospholipid interactions (Arai et al., 1989; Fulcher, 1991; Saenko and Scandella, 1995), and maybe factor VIII-vWF binding as well (Gilles et al., 1999; Saenko et al., 1994). It has also been suggested that inhibitor antibodies may interfere with thrombin cleavage of the factor VIII molecule (Lazarchick et al., 1986). Yet 
another more speculative mechanism is by blocking of its interaction with factor $\mathrm{X}$ and factor IX (Fulcher, 1991; Hoyer 1991). Recent study has confirmed that some factor VIII inhibitors with anti-A3-C1 IgG act by prevention of the FVIIIa/FIXa association (Zhong et al., 1998). In addition, a previously unknown mechanism by which inhibitors may act on FVIII:C was demonstrated to be by causing the proteolysis of factor VIII by inhibitor IgG at the major sites between the A1 and A2 domains (R372-S373); N-terminus of the A3 domain (Y1689-D1681); and E1794-D1795 in the A3 domain (Lacroix-Desmazes et al., 1999). There is as yet no evidence for a relationship between definite inhibitor epitopes and the kinetics of the binding to and inactivation of factor VIII.

Epitope mapping is of therapeutic interest for the future. Mutant factor VIII molecules which contain amino acid substitutions in epitope regions could be synthesised by recombinant DNA methods. The molecules could be designed to be non-reactive with inhibitors. If such antibody-resistant molecules are fully active and have a normal circulating half-life, they could be used to treat the patients effectively.

\section{Chapter Summary}

Epitope detection on the factor VIII peptide is no doubt a critical task for our better knowledge of the immunochemistry of the anti-factor VIII antibodies. Although the methodology remains a challenge, using recombinant DNA technology to produce factor VIII peptides with any desired size still seems to be a reasonable way to carry out a thorough study of epitope determination. Future direction may also include attempts to explain the inactivation mechanisms. Information about the immunobiology of factor VIII inhibitors, mainly the epitope specificity, may help us to understand factor VIII inhibitor 
formation in the same way that investigators are beginning to understand the pathogenesis of other autoantibody diseases (Hoyer, 1991). 


\section{CHAPTER SIX}

\section{GENERAL DISCUSSION}

Human antibodies that inactivate FVIII:C are detected in approximately $25 \%$ of multitransfused patients with haemophilia A. These inhibitory antibodies (inhibitors) arise as an immune response to infused factor VIII. Less commonly, factor VIII inhibitors also develop as autoantibodies in individuals with previously normal haemostasis. Patients with genotypes (such as inversion, large deletion, and stop codon mutation) associated with the most severe clinical phenotypes pose a greater risk of developing factor VIII inhibitors (Reding et al., 1999). Factor VIII replacement therapy in these cases is less effective or ineffective and clinical management may be exceptionally difficult.

Factor VIII inhibitors have been classified according to their in vitro kinetic behaviour. Generally, type I inhibitors, which are more commonly seen in patients with severe inherited haemophilia, have second-order kinetics and completely inhibit exogenous FVIII:C. Type II inhibitors follow more complex kinetics, and do not completely inhibit FVIII:C (Biggs et al., $1972 \mathrm{a}$ and b; Gawryl and Hoyer, 1982). However, previous studies have all used different methods and criteria to classify inhibitor type. There has been no evaluation of the effectiveness of these different methods to classify inhibitors in a single group of patients.

Biggs et al. (1972 a and b) described linear inactivation on a time course graph as typical for type I kinetics, but their graphical representations are not consistent with this statement. In particular, as well as graphs of type I kinetics that are linear, they also showed graphs that are curvilinear. These latter graphs show a steep decline followed by a continuous 
gradual decline. This is in fact not different from their graph for a typical type II pattern. The descriptions by other authors (Allain and Frommel, 1974; Green et al., 1997) are also inconsistent and contradictory. The only approach which appears straightforward is that described by Gawryl and Hoyer (1982). In their approach, they measured the effect of varying the concentration of inhibitor rather than a time course. We therefore studied inhibitors from a group of patients and examined the effects of incubation time and effect of antibody concentration on the kinetics of inactivation of factor VIII.

The results of the time course study showed that inhibitors from eight out of the nine patients demonstrated a similar pattern of inactivation of FVIII:C, which was characterised by a rapid initial decrease of residual FVIII:C (\%) followed by a gradual continuous decline. In addition, mathematical analysis did not show any features that would allow separation into two groups. Therefore, by this approach all of these eight cases appear the same, and we cannot distinguish whether they have type I or type II kinetics. Only one case (Case 11) showed a slow linear inactivation on the time course graph, which by Biggs' criteria would be type I. However, this case later on was defined to have a type II inhibitor.

In our studies of the in vitro kinetics of factor VIII inhibitors, by the study of the effect of antibody concentration, we were able to divide the 11 patients with haemophilia A into two groups. The classification results were obtained by both visual observation of the antibody concentration graph (using patient plasma or purified and concentrated IgG) and mathematical analysis of the data. These groups corresponded to the two groups described by Gawryl and Hoyer (1982). Five patients with severe inherited haemophilia were all found to have type I inhibitors. Three mild inherited cases were studied, and two with type 
I and one with type II kinetics were found. Three acquired cases were studied and one showed type I kinetics and two showed type II pattern. Therefore, a patient's clinical group does not always determine the kinetic type of their inhibitors. While it may be more common for patients with acquired haemophilia to have type II inhibitors, some patients may have inhibitors with type I characteristics which completely neutralise all factor VIII. Also, in patients with inherited mild haemophilia, either type I or type II inhibitors may be seen. Expanding this study to classify more patients would give a better estimate of the prevalence of the two types of inhibitor in these clinical groups.

Hence studying the effect of antibody concentration provides a straightforward approach to the classification of inhibitors. By contrast, study of the time course was in our patients relatively non-productive. Nevertheless, we also studied the time course by an alternative approach in four patients. After incubating factor VIII with inhibitor for four hours, further factor VIII is added to the incubation mixture. In Biggs' studies a type II inhibitor will inhibit this additional factor VIII whereas a type I inhibitor will not. Biggs interpreted this as evidence for weaker binding of type II inhibitors. We were able to demonstrate this phenomenon in patients who had been classified as type II by study of antibody dilution, but not in those who were type I. This is therefore consistent with Biggs' finding. However, this approach did not provide a straightforward way to distinguish the inhibitors. This is because the interpretation is dependent on obtaining a critical ratio of factor VIII to inhibitor in the incubation mixture.

Our study has for the first time, used a number of previously described methods (namely, examining both the time course and the effect of antibody concentration) in a relatively 
large group of patients, in order to assess these two methods for classification of their inhibitor type. Our conclusion is that study of the time course does not provide a means of readily classifying these patients. Therefore, for a standard approach to the classification of factor VIII inhibitors based on in vitro kinetics, we recommend studying the effect of antibody concentration. A plasma dilution study is suitable for high-titre inhibitors $(>10$ $\mathrm{BU} / \mathrm{ml})$. A type I inhibitor is characterised by a steep slope and complete $(>98 \%)$ inactivation of FVIII:C at higher antibody concentrations, while a type II inhibitor gives a plateau and incomplete inactivation even at high antibody concentrations. Purified and concentrated IgG is helpful for the study of antibodies which are not present at high enough levels in plasma. Mathematical analysis would strengthen confidence in the interpretation made by visual observation of the curves.

In our studies we have examined only 11 patients. It would be of interest to expand this work to include the application of this approach to a larger number of patients, covering as many variables as possible, i.e., patients with different clinical severity, different gene mutations, different replacement therapy (e.g., children receiving recombinant factor VIII, and adult patients receiving AHF), and patients from a wider range of sources, e.g., patients from different regions, and different racial groups. It would be important to study both the time course and the effect of varying antibody concentration. Although the study of the time course was not fruitful in our study, examining a large number of patients may disclose the presence of different types of time course. In particular, it is of interest to determine whether or not there is a small group with a linear time course such as that described by Biggs and found in only one of our patients. 
There are two possible hypotheses to explain the complex kinetics of type II inhibitors. One is that type II inhibitors have relatively low affinity binding to the factor VIII molecule (Allain and Frommel, 1974; Gilles et al., 1997; Hoyer and Gawryl, 1984; Kasper, 1984). It is therefore possible for them to dissociate from the original factor VIII molecules which remain inactivated, and bind to additional factor VIII molecules which also become inactivated. This explains the effect on an extra amount of factor VIII added during the time course experiments (Figure 3.9). Another hypothesis for the type II kinetics is that these inhibitors only partially inhibit FVIII:C. This could, occur for instance, if they blocked only part of an important functional site. Such a hypothesis explains the plateau (incomplete inactivation) on the antibody concentration curve even when inhibitor is present at high concentration. However, this does not explain their ability to inactivate an extra source of factor VIII added after equilibrium is apparently reached.

Inhibitory antibodies bind to one or more of the factor VIII epitopes which are essential for procoagulant activity. Epitopes recognised by factor VIII inhibitors have been mapped to three main clusters located in the $\mathrm{A} 2, \mathrm{~A} 3$ and $\mathrm{C} 2$ domains on factor VIII molecule (Lollar, 1999). The majority of factor VIII inhibitors are directed against one of the following areas:

1) the carboxyl end of the $\mathrm{C} 2$ domain, which contains phospholipid and vWF binding sites; 2) the amino end of the A2 domain, which is responsible for the interaction with factor $\mathrm{Xa}$; 3) the amino end of the A3 domain, which contains a factor IX binding site (Reding et al., 1999). It is possible that the complete inactivation of FVIII:C by type I inhibitors may be caused by the complete blockage of any of those important functional regions. For type II 
inhibitors, the incomplete inactivation of FVIII:C may be due to partial blockage of those regions.

Inhibitor neutralisation studies indicate that the effects of anti-A2, anti-A3, and anti-C2 antibodies on the Bethesda titre are additive (Prescott et al., 1997). Thus, inhibitors to a given domain appear to act independently of inhibitors to other domains. Haemophilia inhibitors and autoantibodies have the same broad domain specificity, which is somewhat surprising given the different immunologic settings in which they arise (Lollar, 1999).

There is evidence that a proportion of anti-factor VIII antibodies do not inhibit the procoagulant function of the molecule (Gilles et al., 1993). This conclusion was based on an apparent lack of correlation between inhibition of function as shown by a chromogenic bioassay method, and amount of antibody bound to factor VIII. These antibodies have been referred to as non-inhibitory antibodies. Such antibodies may act against nonfunctional epitopes in a patient in whom there is no inhibitor against a functional epitope. It has been suggested that they may play a role in increasing the clearance of factor VIII from the circulation (Kessler, 1991; Nilsson et al., 1990). This could be by increasing the uptake of factor VIII-immunoglobulin complexes by phagocytic cells of the reticuloendothelial system (Gilles et al., 1997). The ELISA method is one immunoassay approach that has been developed, aiming to detect both the inhibitory and non-inhibitory anti-factor VIII antibodies (Dazzi et al., 1996).

As part of this study an ELISA method to detect factor VIII inhibitors was established. One way to obtain evidence for non-inhibitory antibodies is to study patients with 
haemophilia A who are negative for inhibitors by the Bethesda assay. Should these patients be positive by ELISA, this would suggest that there is antibody binding to epitopes that are not important for function. Our results by ELISA showed a similar sensitivity between the ELISA method and the Bethesda assay for detection of factor VIII antibodies in 50 of our patients with haemophilia A. Twenty-three out of the 24 haemophilia patients with a positive Bethesda titre showed a positive ELISA result, while none of the 26 patients with a negative Bethesda titre was found to have a positive result by ELISA. In addition, there was a definite correlation between the OD value of the ELISA method and the Bethesda titre in these 50 patients.

In contrast to two previous published reports (Dazzi et al., 1996; Vianello et al., 1997), we did not detect inhibitors by ELISA in any patients who did not have an inhibitor as detected by the Bethesda method. Thus, in the patient group studied here, non-inhibitory factor VIII antibodies are not a consequence of treatment with factor VIII replacement therapy. Therefore, non-inhibitory factor VIII antibodies may be rarer in this Australian patient group than in European studies. One possible reason might be because haemophilia patients in Australia are treated with plasma-derived AHF exclusively from one manufacturer (CSL). This may have an effect on the antigenicity of the factor VIII molecule by inducing an inhibitor with a different epitope specificity to that in the European patients where factor VIII concentrate from several manufacturers is used. Another reason may be the different sources of factor VIII used to coat the microtitre plates in different studies, which may lead to the exposure of different epitopes on the factor VIII molecule. 
In our group of patients studied by ELISA, a reasonable number $(n=20)$ of patients have the inversion mutation. Intron 22 gene inversion is the most common mutation in inherited haemophilia A with a severe phenotype. It was of interest whether this particular mutation would lead to findings different to those found in a group of severe patients with other gene defects. Our study showed that both ELISA and the Bethesda assay gave a similar proportion of positive results in severe patients with (40\% and $40 \%$, respectively) and without $(38 \%$ and $46 \%)$ the inversion. The prevalence $(40 \%)$ of inhibitors by Bethesda assay in our severe patient group with intron 22 inversion is similar to that in other studies (34.4\% in the study by Becker et al., 1996 and 35-40\% in the study by Tuddenham and McVey, 1998). There were differences between the prevalence of inhibitors in our study compared with the European study (Vianello et al., 1997). In their study, $81 \%$ of patients with severe inherited haemophilia with intron 22 gene inversion, were found to have positive ELISA results, while only $25 \%$ of them had detectable inhibitor by the Bethesda assay.

A future prospective survey on a larger group of randomly selected patients may better evaluate the applicability of the ELISA method. It will also be useful to carry out the ELISA tests using different factor VIII source to coat the plates. Currently we do not know the clinical importance of a positive ELISA result in a patient with a negative Bethesda titre. It will only be important if binding of the antibody to the factor VIII molecule leads to an increase in clearance and therefore a reduction in survival time.

The epitopes which characterise individual inhibitors may be numerous, as the human immune response towards factor VIII is highly heterogeneous (Hoyer, 1991). It was 
suggested that type I inhibitors may interact with different epitopes as compared to type II inhibitors (Biggs et al., 1972 a and b; Gawryl and Hoyer, 1982). Patients with type I inhibitors have both anti-A2 and anti-C2 antibodies, possibly in addition to some other epitopes on the light chain, such as in the A3 domain (Prescott et al., 1997). On the contrary, acquired haemophilia patients with type II inhibitors tend to have a simpler antibody repertoire, with their antibodies more likely to be specific for a single domain (A2 or C2), but not both (Prescott et al., 1997). It is important to study larger group of patients to correlate epitopes and kinetics.

Methods used to identify antibody binding sites (epitopes) include competition with monoclonal antibodies of known specificity, use of cDNA-derived (recombinant) factor VIII fragments, hybrids of human and porcine factor VIII, and synthetic peptides. All but the first of these methods make use of the factor VIII protein or fragments thereof, but do not account for the fact that factor VIII antigenicity could be altered by conformational changes, which are known to occur upon factor VIII interaction with vWF, or as the result of proteolytic cleavage by thrombin (Gilles et al., 1999).

In this study thrombin-cleaved fragments of recombinant factor VIII protein were generated with the aim of determining heavy and light chain specificity of inhibitors. Whilst successful with a monoclonal antibody model the approach was unsuccessful for the study of IgG purified from patient plasma. This is likely due to insufficient sensitivity of the system for the relatively low levels of anti-factor VIII antibody tested $(\sim 7-40 \mathrm{BU} / \mathrm{ml}$ from patient IgG concentrates). Further directions for this work could include the use of a more sensitive detection system in the immunoblotting method (e.g., streptavidin-biotin or 
$\mathrm{I}^{125}$ labelled secondary anti-human antibody). Also, the production of recombinant factor VIII fragments would allow more precise localisation of epitopes.

In further studies it would be of great interest to investigate the possible relationship between the gene defect, epitope specificity, and the clinical and kinetic behaviour of the factor VIII inhibitors. Results of such studies may give additional information about the mechanisms of antibody formation and their inactivation of FVIII:C, which may lead not only to more informed and effective therapeutic approaches for haemophilia patients with inhibitors, but also to a better understanding of the human immune response. 


\section{REFERENCES}

1. Aggeler PM, White SG, Glendening MB, Page EW, Leake TB, Bates G. Plasma thromboplastin component (PTC) deficiency: A new disease resembling hemophilia. Proc Soc Exp Biol Med 1952; 79:692-4.

2. Aledort LM. The cause of death in hemophiliacs. National heart, lung and blood institute: unsolved therapeutic problems in hemophilia. Washington DC: US Government Printing Office, 1976.

3. Aledort LM. Hemophilia: yesterday, today, and tomorrow. Mt Sinai J Med 1996; 63:225-35.

4. Allain JP, Frommel D. Antibodies to factor VIII: specificity and kinetics of iso- and hetero- antibodies in hemophilia A. Blood 1974; 44:313-22.

5. Andersen BR, Troup SB. Gamma-G-antibody to human anti-hemophilic globulin (factor 8). J Immunol 1968; 100:175-86.

6. Antonarakis SE, Rossiter JP, Young M, et al. Factor VIII gene inversions in severe hemophilia A: results of an international consortium study. Blood 1995; 86:220612.

7. Arai M, Scandella D, Hoyer LW. Molecular basis of factor VIII inhibition by human antibodies. Antibodies that bind to the factor VIII light chain prevent the interaction of factor VIII with phospholipid. J Clin Invest 1989; 83:1978-84.

8. Becker J, Schwaab R, Moller-Taube A, et al. Characterization of the factor VIII defect in 147 patients with sporadic hemophilia A: family studies indicate a mutation type- dependent sex ratio of mutation frequencies. Am $\mathrm{J}$ Hum Genet $1996 ; 58: 657-70$.

9. Berntorp E, Boulyjenkov V, Brettler D, et al. Modern treatment of haemophilia. Bull World Health Organ 1995; 73:691-701.

10. Biggs R, Bidwell E. A method for the study of antihaemophilic globulin inhibitors with reference to six cases. Br J Haemat 1959; 5:379-95.

11. Biggs R, Austen DE, Denson KW, Rizza CR, Borrett R. The mode of action of antibodies which destroy factor VIII. I. Antibodies which have second-order concentration graphs. Br J Haematol 1972 a; 23:125-35.

12. Biggs R, Austen DE, Denson KW, Borrett R, Rizza CR. The mode of action of antibodies which destroy factor VIII. II. Antibodies which give complex concentration graphs. Br J Haematol 1972 b; 23:137-55.

13. Biggs R. Haemophilia treatment in the United Kingdom from 1969 to 1974 . Br J Haematol 1977; 35:487-504. 
14. Bloom AL. The management of patients with inherited blood coagulation disorders. In: Bloom AL, Forbes CD, Thomas DP, Tuddenham EGD, eds. Haemostasis and thrombosis. Vol. 2. New York: Churchill Livingstone Inc., 1994:897-917.

15. Bovill EG, Burns SL, Golden EA. Factor VIII antibody in a patient with mild haemophilia. Br J Haematol 1985; 61:323-8.

16. Bray GL, Gomperts ED, Courter S, et al. A multicenter study of recombinant factor VIII (recombinate): safety, efficacy, and inhibitor risk in previously untreated patients with hemophilia A. The Recombinate Study Group. Blood 1994; 83:242835.

17. Brettler DB, Levine PH. Clinical manifestations and therapy of inherited coagulation factor deficiencies. In: Colman RW, Hirsh J, Marder VJ, Salzman EW, eds. Hemostasis and thrombosis: basic principles and clinical practice. Philadelphia: J.B. Lippincott Company, 1993:169-83.

18. Brettler DB. Inhibitors of factor VIII and IX. Haemophilia 1995; 1:35-9.

19. Dazzi F, Tison T, Vianello F, et al. High incidence of anti-FVIII antibodies against non-coagulant epitopes in haemophilia A patients: a possible role for the half-life of transfused FVIII. Br J Haematol 1996; 93:688-93.

20. Ehrenforth S, Kreuz W, Scharrer I, et al. Incidence of development of factor VIII and factor IX inhibitors in haemophiliacs [see comments]. Lancet 1992; 339:594-8.

21. Ehrenforth S, Kreuz W, Scharrer I, Kornhuber B. Factor VIII inhibitors in haemophiliacs [letter; comment]. Lancet 1992; 340:253.

22. Ehrlich HJ, Bray GL, Gomperts ED. Comparison of high responder inhibitor frequency in recent studies of previously untreated patients with hemophilia A [letter]. Thromb Haemost 1998; 79:242-3.

23. Eyster ME, Gill FM, Blatt PM, Hilgartner MW, Ballard JO, Kinney TR. Central nervous system bleeding in hemophiliacs. Blood 1978; 51:1179-88.

24. Feinstein DI. Immune coagulation disorder. In: Colman RW, Hirsh J, Marder VJ, Salzman EW, eds. Hemostasis and thrombosis: basic principles and clinical practice. Philadelphia: J.B. Lippincott Company, 1993:881-905.

25. Fowler WE, Fay PJ, Arvan DS, Marder VJ. Electron microscopy of human factor V and factor VIII: correlation of morphology with domain structure and localization of factor V activation fragments. Proc Natl Acad Sci U S A 1990; 87:7648-52.

26. Fulcher CA, de Graaf Mahoney S, Roberts JR, Kasper CK, Zimmerman TS. Localization of human factor FVIII inhibitor epitopes to two polypeptide fragments. Proc Natl Acad Sci U S A 1985; 82:7728-32.

27. Fulcher CA, de Graaf Mahoney S, Zimmerman TS. FVIII inhibitor IgG subclass and FVIII polypeptide specificity determined by immunoblotting. Blood 1987; 69:1475-80. 
28. Fulcher CA, Lechner K, de Graaf Mahoney S. Immunoblot analysis shows changes in factor VIII inhibitor chain specificity in factor VIII inhibitor patients over time. Blood 1988; 72:1348-56.

29. Fulcher CA. Immunochemistry of factor VIII:C inhibitor antibodies. Am J Med 1991; 91:6S-8S.

30. Furie B, Limentani SA, Rosenfield CG. A practical guide to the evaluation and treatment of hemophilia. Blood 1994; 84:3-9.

31. Furie B, Furie BC. Molecular basis of blood coagulation. In: Hoffman R, Benz EJ, Jr, Shattil SJ, Furie B, Cohen HJ, Silberstein LE, eds. Hematology: basic principles and practice. New York: Churchill Livingstone Inc., 1995:1566-87.

32. Gautier P, Sultan Y, Parquet-Gernez A, Meriane F, Guerois C, Derlon A. Detection and IgG subclass analysis of antibodies to factor VIII in multitransfused haemophiliacs and healthy individuals. Haemophilia 1996; 2:88-94.

33. Gawryl MS, Hoyer LW. Inactivation of factor VIII coagulant activity by two different types of human antibodies. Blood 1982; 60:1103-9.

34. Giles AR, Verbruggen B, Rivard GE, Teitel J, Walker I. A detailed comparison of the performance of the standard versus the Nijmegen modification of the Bethesda assay in detecting factor VIII:C inhibitors in the haemophilia A population of Canada. Association of Hemophilia Centre Directors of Canada. Factor VIII/IX Subcommittee of Scientific and Standardization Committee of International Society on Thrombosis and Haemostasis. Thromb Haemost 1998; 79:872-5.

35. Gill JC. The role of genetics in inhibitor formation. Thromb Haemost 1999; 82:500-504.

36. Gilles JG, Arnout J, Vermylen J, Saint-Remy JM. Anti-factor VIII antibodies of hemophiliac patients are frequently directed towards nonfunctional determinants and do not exhibit isotypic restriction. Blood 1993; 82:2452-61.

37. Gilles JG, Desqueper B, Lenk H, Vermylen J, Saint-Remy JM. Neutralizing antiidiotypic antibodies to factor VIII inhibitors after desensitization in patients with hemophilia A. J Clin Invest 1996; 97:1382-8.

38. Gilles JG, Jacquemin MG, Saint-Remy JM. Factor VIII inhibitors. Thromb Haemost 1997; 78:641-6.

39. Gilles JG, Lavend'homme R, Peerlinck K, et al. Some factor VIII (FVIII) inhibitors recognise a FVIII epitope(s) that is present only on FVIII-vWF complexes. Thromb Haemost 1999; 82:40-5.

40. Goldsmith JC. Diagnosis of factor VIII versus nonspecific inhibitors. Semin Hematol 1993; 30:3-6. 
41. Goodeve AC, Preston FE, Peake IR. Factor VIII gene rearrangements in patients with severe haemophilia A [see comments]. Lancet 1994; 343:329-30.

42. Gordon EM, Mungo R, Goldsmith JC. Lingual hemorrhage in a patient with hemophilia A complicated by a high titer inhibitor. Management by continuous infusion of monoclonal antibody-purified factor VIII [see comments]. Am J Pediatr Hematol Oncol 1993; 15:107-10.

43. Gordon EM, al-Batniji F, Goldsmith JC. Continuous infusion of monoclonal antibody-purified factor VIII: rational approach to serious hemorrhage in patients with allo- /autoantibodies to factor VIII. Am J Hematol 1994; 45:142-5.

44. Green D. Spontaneous inhibitors of factor VIII. Br J Haemat 1968; 15:57-75.

45. Green D, Lechner K. A survey of 215 non-hemophilic patients with inhibitors to Factor VIII. Thromb Haemost 1981; 45:200-3.

46. Green D, Blanc J, Foiles N. Spontaneous inhibitors of fctor VIII: Kinetics of inactivation of human and porcine factor VIII. J Lab Clin Med 1999; 133:260-4.

47. Handin RI. Bleeding and thrombosis. In: Fauci AS, Martin JB, Braunwald E, et al., eds. Harrison's principles of internal medicine. Vol. 1. New York: The McGrawHill Companies, Inc., 1998 a:339-45.

48. Handin RI. Disorders of coagulation and thrombosis. In: Fauci AS, Martin JB, Braunwald E, et al., eds. Harrison's principles of internal medicine. Vol. 1. New York: The McGraw-Hill Companies, Inc., 1998 b:736-43.

49. Hay CR, Ollier W, Pepper L, et al. HLA class II profile: a weak determinant of factor VIII inhibitor development in severe haemophilia A. UKHCDO Inhibitor Working Party. Thromb Haemost 1997; 77:234-7.

50. Hay CR, Ludlam CA, Colvin BT, et al. Factor VIII inhibitors in mild and moderate-severity haemophilia A. UK Haemophilia Centre Directors Organisation [see comments]. Thromb Haemost 1998; 79:762-6.

51. Hay CR. Factor VIII inhibitors in mild and moderate-severity haemophilia A. Haemophilia 1998; 4:558-63.

52. Healey JF, Lubin IM, Nakai H, et al. Residues 484-508 contain a major determinant of the inhibitory epitope in the A2 domain of human factor VIII. J Biol Chem 1995; 270:14505-9.

53. Hedner U, Glazer S, Falch J. Recombinant activated factor VII in the treatment of bleeding episodes in patients with inherited and acquired bleeding disorders. Trans Med Rev 1993; 7:78-83.

54. Hoyer LW. The factor VIII complex: structure and function. Blood 1981; 58:1-13.

55. Hoyer LW, Gawryl MS, de la Fuente B. Immunochemical characterization of factor VIII inhibitors. Prog Clin Biol Res 1984; 150:73-85. 
56. Hoyer LW. Immunochemical properties of factor VIII and factor IX inhibitors. Blood Coagulation and Fibrinolysis 1991; 2:11-15.

57. Hoyer LW. Hemophilia A. N Engl J Med 1994; 330:38-47.

58. Hoyer LW, Scandella D. Factor VIII inhibitors: structure and function in autoantibody and hemophilia A patients. Semin Hematol 1994; 31:1-5.

59. Hoyer LW. Acquired anticoagulants. In: Beutler E, Lichtman MA, Coller BS, Kipps TJ, eds. Williams Hematology. New York: McGraw-Hill, Inc., 1995:14851496.

60. Ingram G. The history of haemophilia. Haemophilia 1997; 3:5-15.

61. Jesty J, Nemerson Y. The pathway of blood coagulation. In: Beutler E, Lichtman MA, Coller BS, Kipps TJ, eds. Williams Hematology. New York: McGraw-Hill, Inc., 1995:1227-38.

62. Kasper CK, Aledort L, Aronson D, et al. Proceedings: A more uniform measurement of factor VIII inhibitors. Thromb Diath Haemorrh 1975; 34:869-72.

63. Kasper CK. Measurement of factor VIII inhibitors. Prog Clin Biol Res 1984; 150:87-98.

64. Kasper CK. Complications of hemophilia A treatment: factor VIII inhibitors. Ann N Y Acad Sci 1991; 614:97-105.

65. Kaufman RJ, Wasley LC, Davies MV, Wise RJ, Israel DI, Dorner AJ. Effect of von Willebrand factor coexpression on the synthesis and secretion of factor VIII in Chinese hamster ovary cells. Mol Cell Biol 1989; 9:1233-42.

66. Kaufman PJ, Antonarakis SE. Structure, biology, and genetics of factor VIII. In: Hoffman R, Benz EJ, Jr, Shattil SJ, Furie B, Cohen HJ, Silberstein L, eds. Hematology: basic principles and practice. New York: Churchill Livingstone Inc., 1995:1633-91.

67. Kavanagh ML, Wood CN, Davidson JF. The immunological characterization of human antibodies to factor VIII isolated by immuno-affinity chromatography. Thromb Haemost 1981; 45:60-4.

68. Kemball-Cook G, Tuddenham EG. The Factor VIII Mutation Database on the World Wide Web: the haemophilia A mutation, search, test and resource site. HAMSTeRS update (version 3.0). Nucleic Acids Res 1997; 25:128-32.

69. Kernoff PB. The relevance of factor VIII inactivation characteristics in the treatment of patients with antibodies directed against factor VIII. Br J Haematol $1972 ; 22: 735-42$.

70. Kessler CM. An introduction to factor VIII inhibitors: the detection and quantitation. Am J Med 1991; 91:1S-5S. 
71. Lacroix-Desmazes S, Moreau A, Sooryanarayana, et al. Catalytic activity of antibodies against factor VIII in patients with hemophilia A. Nat Med 1999; 5:1044-7.

72. Lakich D, Kazazian HH, Jr., Antonarakis SE, Gitschier J. Inversions disrupting the factor VIII gene are a common cause of severe haemophilia A [see comments]. Nat Genet 1993; 5:236-41.

73. Lazarchick J, Ashby MA, Lazarchick JJ, Sens DA. Mechanism of factor VIII inactivation by human antibodies. IV. Antibody binding prevents factor VIII proteolysis by thrombin. Ann Clin Lab Sci 1986; 16:497-501.

74. Leitner A, Bidwell E, Dike GWR. An antihaemophilic globulin (Factor VIII) inhibitor: purification, characterization and reaction kinetics. Br J Haemat 1963; 9:245-58.

75. Levine PH. Clinical manifestations and therapy of hemophilia A and B. In: Colman RW, Hirsh J, Marder VJ, Salzman EW, eds. Hemostasis and thrombosis: basic priciples and clinical practice. Philadelphia: J.B. Lippincott Company, 1987:97111.

76. Ljung RC. Intron 22 inversions and haemophilia [letter; comment]. Lancet 1994; 343:791.

77. Lloyd JV, Street AM, Berry E, et al. Cross-reactivity to porcine factor VIII of factor VIII inhibitors in patients with haemophilia in Australia and New Zealand. Aust N Z J Med 1997; 27:658-64.

78. Lollar P. Analysis of factor VIII inhibitors using hybrid human/porcine factor VIII. Thromb Haemost 1997; 78:647-51.

79. Lollar P. Characterization of factor VIII B-cell inhibitory epitopes. Thromb Haemost 1999; 82:505-8.

80. Lozier JN, High KA. Molecular basis of hemophilia. Hematol Pathol 1990; 4:1-26.

81. Lubin IM, Healey JF, Scandella D, Runge MS, Lollar P. Elimination of a major inhibitor epitope in factor VIII. J Biol Chem 1994; 269:8639-41.

82. Luddington R, Jennings I. Factor VIIIc inhibitors: an automated assay. Med Lab Sci 1992; 49:135-7.

83. Lusher JM. Factor VIII inhibitors. Etiology, characterization, natural history, and management. Ann N Y Acad Sci 1987; 509:89-102.

84. Lusher JM, Arkin S, Abildgaard CF, Schwartz RS. Recombinant factor VIII for the treatment of previously untreated patients with hemophilia A. Safety, efficacy, and development of inhibitors. Kogenate Previously Untreated Patient Study Group. N Engl J Med 1993; 328:453-9. 
85. Lusher JM. Acquired inhibitors to factor VIII in non-hemophilic patients. In: Kessler C, Garvey MB, Green D, Kasper C, Lusher J, eds. Acquired hemophilia. Princeton: Excerpta Medica, Inc., 1995:1-8.

86. Mann KG, Nesheim ME, Church WR, Haley P, Krishnaswamy S. Surfacedependent reactions of the vitamin K-dependent enzyme complexes. Blood 1990; 76:1-16.

87. Mann KG. Biochemistry and physiology of blood coagulation. Thromb Haemost $1999 ; 82: 165-74$.

88. Mannucci PM, Tripodi A. Factor VIII clotting activity. In: Jespersen J, Bertina RM, Haverkate F, eds. ECAT assay procedures: a manual of laboratory techniques. Dordrecht: Kluwer Academic Publishers, 1992:65-70.

89. Mannucci PM, Giangrande PLF. Acquired disorders of coagulation. In: Bloom AL, Forbes CD, Thomas DP, Tuddenham EGD, eds. Haemostasis and thrombosis. Vol. 2. New York: Churchill Livingstone Inc., 1994:949-68.

90. McMillan CW, Shapiro SS, Whitehurst D, Hoyer LW, Rao AV, Lazerson J. The natural history of factor VIII:C inhibitors in patients with hemophilia A: a national cooperative study. II. Observations on the initial development of factor VIII:C inhibitors. Blood 1988; 71:344-8.

91. Michiels JJ, Hamulyak K, Nieuwenhuis HK, Novakova I, van Vliet HH. Acquired haemophilia $\mathrm{A}$ in women postpartum: management of bleeding episodes and natural history of the factor VIII inhibitor. Eur J Haematol 1997; 59:105-9.

92. Mondorf W, Ehrenforth S, Vigh Z, et al. Screening of F.VIII:C antibodies by an enzyme-linked immunosorbent assay. Vox Sang 1994; 66:8-13.

93. Mosher DF. Disorders of blood coagulation. In: Bennett JC, Plum F, eds. Cecil textbook of medicine. Philadelphia: W.B. Saunders Company, 1996:987-1003.

94. Nilsson IM, Berntorp E, Zettervall O, Dahlback B. Noncoagulation inhibitory factor VIII antibodies after induction of tolerance to factor VIII in hemophilia A patients. Blood 1990; 75:378-83.

95. Oldenburg J, Picard JK, Schwaab R, Brackmann HH, Tuddenham EG, Simpson E. HLA genotype of patients with severe haemophilia A due to intron 22 inversion with and without inhibitors of factor VIII. Thromb Haemost 1997; 77:238-42.

96. Pavlovsky A. Contribution to the pathogenesis of hemophilia. Blood 1947; 2:18591.

97. Peerlinck K, Arnout J, Gilles JG, Saint-Remy JM, Vermylen J. A higher than expected incidence of factor VIII inhibitors in multitransfused haemophilia A patients treated with an intermediate purity pasteurized factor VIII concentrate [see comments]. Thromb Haemost 1993; 69:115-8. 
98. Peerlinck K, Arnout J, Di Giambattista M, et al. Factor VIII inhibitors in previously treated haemophilia A patients with a double virus-inactivated plasma derived factor VIII concentrate. Thromb Haemost 1997; 77:80-6.

99. Pool JG. High-potency antihaemophilic factor concentrate prepared from cryoglobulin in precipitate. Nature 1964; 203:312.

100. Pool JG, Miller RG. Assay of the immune inhibitor in classic haemophilia: application of virus-antibody reaction kinetics. Br J Haematol 1972; 22:517-28.

101. Prescott R, Nakai H, Saenko EL, et al. The inhibitor antibody response is more complex in hemophilia A patients than in most nonhemophiliacs with factor VIII autoantibodies. Blood 1997; 89:3663-71.

102. Pruthi RK, Nichols WL. Autoimmune factor VIII inhibitors. Curr Opin Hematol $1999 ; 6: 314-22$.

103. Reding $\mathrm{MT}, \mathrm{Wu} \mathrm{H}, \mathrm{Krampf} \mathrm{M}$, et al. CD4+ $\mathrm{T}$ cell response to factor VIII in hemophilia A, acquired hemophilia, and healthy subjects [In Process Citation]. Thromb Haemost 1999; 82:509-15.

104. Regnault V, Stoltz JF. Quantitation of factor VIII antibodies by an enzyme-linked immunoassay method [letter]. Blood 1994; 83:1155-6.

105. Rizza CR, Biggs R. The treatment of patients who have factor-VIII antibodies. $\mathrm{Br} \mathrm{J}$ Haematol 1973; 24:65-82.

106. Roberts HR, Hoffman M. Hemophilia and related conditions- inherited deficiencies of prothrombin (factor II), factor V, and factor VII to XII. In: Beutler E, Lichtman MA, Coller BS, Kipps TJ, eds. Williams Hematology. New York: McGraw-Hill, Inc., 1995:1413-39.

107. Rosendaal FR, Smit C, Briet E. Hemophilia treatment in historical perspective: a review of medical and social developments. Ann Hematol 1991; 62:5-15.

108. Rosendaal FR, Nieuwenhuis HK, van den Berg HM, et al. A sudden increase in factor VIII inhibitor development in multitransfused hemophilia A patients in The Netherlands. Dutch Hemophilia Study Group. Blood 1993; 81:2180-6.

109. Saenko EL, Shima M, Rajalakshmi KJ, Scandella D. A role for the C2 domain of factor VIII in binding to von Willebrand factor. J Biol Chem 1994; 269:11601-5.

110. Saenko EL, Scandella D. A mechanism for inhibition of factor VIII binding to phospholipid by von Willebrand factor. J Biol Chem 1995; 270:13826-33.

111. Saenko EL, Shima M, Gilbert GE, Scandella D. Slowed release of thrombincleaved factor VIII from von Willebrand factor by a monoclonal and a human antibody is a novel mechanism for factor VIII inhibition. J Biol Chem 1996; $271: 27424-31$.

112. Sallah S. Inhibitors to clotting factors. Ann Hematol 1997; 75:1-7. 
113. Sanchez-Cuenca JM, Carmona E, Villanueva MJ, Aznar JA. Immunological characterization of factor VIII inhibitors by a sensitive micro-ELISA method. Thromb Res 1990; 57:897-908.

114. Santagostino E, Gringeri A, Tagliavacca L, Mannucci PM. Inhibitors to factor VIII in a family with mild hemophilia: molecular characterization and response to factor VIII and desmopressin. Thromb Haemost 1995; 74:619-21.

115. Scandella D, DeGraaf Mahoney S, Mattingly M, Roeder D, Timmons L, Fulcher CA. Epitope mapping of human factor VIII inhibitor antibodies by deletion analysis of factor VIII fragments expressed in Escherichia coli. Proc Natl Acad Sci U S A $1988 ; 85: 6152-6$.

116. Scandella D, Mattingly M, de Graaf S, Fulcher CA. Localization of epitopes for human factor VIII inhibitor antibodies by immunoblotting and antibody neutralization. Blood 1989; 74:1618-26.

117. Scandella D, Timmons L, Mattingly M, Trabold N, Hoyer LW. A soluble recombinant factor VIII fragment containing the A2 domain binds to some human anti-factor VIII antibodies that are not detected by immunoblotting. Thromb Haemost 1992; 67:665-71.

118. Scandella D, Gilbert GE, Shima M, et al. Some factor VIII inhibitor antibodies recognize a common epitope corresponding to $\mathrm{C} 2$ domain amino acids 2248 through 2312, which overlap a phospholipid-binding site. Blood 1995 a; 86:1811-9.

119. Scandella D, Kessler C, Esmon P, et al. Epitope specificity and functional characterization of factor VIII inhibitors. Adv Exp Med Biol 1995 b; 386:47-63.

120. Scandella D. Human anti-factor VIII antibodies: epitope localization and inhibitory function. Vox Sang 1996; 70:9-14.

121. Scandella D, Mondorf W, Klinge J. The natural history of the immune response to exogenous factor VIII in severe haemophilia A. Haemophilia 1998; 4:546-51.

122. Schwaab R, Brackmann HH, Meyer C, et al. Haemophilia A: mutation type determines risk of inhibitor formation. Thromb Haemost 1995; 74:1402-6.

123. Shapiro SS. The immunologic character of acquired inhibitors of antihemophilic globulin (factor 8) and the kinetics of their interaction with factor 8. J Clin Invest 1967; 46:147-56.

124. Shapiro SS, Hultin M. Acquired inhibitors to the blood coagulation factors. Semin Thromb Hemost 1975; 1:336-85.

125. Shima M, Scandella D, Yoshioka A, et al. A factor VIII neutralizing monoclonal antibody and a human inhibitor alloantibody recognizing epitopes in the $\mathrm{C} 2$ domain inhibit factor VIII binding to von Willebrand factor and to phosphatidylserine. Thromb Haemost 1993; 69:240-6. 
126. Shima M, Nakai H, Scandella D, et al. Common inhibitory effects of human antiC2 domain inhibitor alloantibodies on factor VIII binding to von Willebrand factor. Br J Haematol 1995; 91:714-21.

127. Shulman NR, Hirschman RJ. Acquired hemophilia. Trans Assoc Am Physicians $1969 ; 82: 388-97$.

128. Shurafa M, Kithier K. A new approach to immunologic identification of factor VIII antibodies. Haemophilia 1995; 1:175-77.

129. Simpson JA, Weiner ESC. The Oxford English Dictionary. In: Murray JAH, H. B, Craigie BWA, T. OC, Burchfield RW, eds. The Oxford English Dictionary. Vol. VI. Oxford: Oxford University Press, 1989:1009.

130. Tizzano EF, Domenech M, Altisent C, Tusell J, Baiget M. Inversions in the factor VIII gene in Spanish hemophilia A patients [letter]. Blood 1994; 83:3826.

131. Tizzano EF, Domenech M, Baiget M. Inversion of intron 22 in isolated cases of severe hemophilia A. Thromb Haemost 1995; 73:6-9.

132. Tuddenham EG, Cooper DN, Gitschier J, et al. Haemophilia A: database of nucleotide substitutions, deletions, insertions and rearrangements of the factor VIII gene. Nucleic Acids Res 1991; 19:4821-33.

133. Tuddenham EG, Schwaab R, Seehafer J, et al. Haemophilia A: database of nucleotide substitutions, deletions, insertions and rearrangements of the factor VIII gene, second edition [corrected and republished in Nucleic Acids Res 1994 Nov 11;22(22):4851- 68]. Nucleic Acids Res 1994; 22:3511-33.

134. Tuddenham EG, McVey JH. The genetic basis of inhibitor development in haemophilia A. Haemophilia 1998; 4:543-5.

135. van Dieijen G, Tans G, Rosing J, Hemker HC. The role of phospholipid and factor VIIIa in the activation of bovine factor X. J Biol Chem 1981; 256:3433-42.

136. Verbruggen B, Novakova I, Wessels H, Boezeman J, van den Berg M, MauserBunschoten E. The Nijmegen modification of the Bethesda assay for factor VIII:C inhibitors: improved specificity and reliability. Thromb Haemost 1995; 73:247-51.

137. Vermylen J. How do some haemophiliacs develop inhibitors? Haemophilia 1998; 4:538-42.

138. Vianello F, Radossi P, Tison T, et al. Prevalence of anti-FVIII antibodies in severe haemophilia A patients with inversion of intron 22. Br J Haematol 1997; 97:807-9.

139. Wacey AI, Kemball-Cook G, Kazazian HH, et al. The haemophilia A mutation search test and resource site, home page of the factor VIII mutation database: HAMSTeRS. Nucleic Acids Res 1996; 24:100-2. 
140. Ware J, MacDonald MJ, Lo M, de Graaf S, Fulcher CA. Epitope mapping of human factor VIII inhibitor antibodies by site- directed mutagenesis of a factor VIII polypeptide. Blood Coagul Fibrinolysis 1992; 3:703-16.

141. Weiss HJ, Sussman, II, Hoyer LW. Stabilization of factor VIII in plasma by the von Willebrand factor. Studies on posttransfusion and dissociated factor VIII and in patients with von Willebrand's disease. J Clin Invest 1977; 60:390-404.

142. White GC, II. Factor VIII inhibitor assay: quantitative and qualitative assay limitations and development needs. Semin Hematol 1994; 31:6-10.

143. Wood WI, Capon DJ, Simonsen CC, et al. Expression of active human factor VIII from recombinant DNA clones. Nature 1984; 312:330-7.

144. Wright IS. The nomenclature of blood clotting factors. Thromb Diath Haemost $1962 ; 7: 381$.

145. Zhong D, Saenko EL, Shima M, Felch M, Scandella D. Some human inhibitor antibodies interfere with factor VIII binding to factor IX. Blood 1998; 92:136-42. 